Supplementary Information for

\title{
Porous Nanosheet Assembly for Macrocyclzation and Self-Release
}

Xin Liu, ${ }^{\dagger, \perp}$ Xiaobin Zhou, ${ }^{\dagger, \perp}$ Bowen Shen, ${ }^{\dagger}$ Yongju Kim,,${ }^{\dagger}$ Huaxin Wang, ${ }^{\dagger}$ Wanting Pan,$\dagger$ Jehan Kim, ${ }^{\S}$ and Myongsoo Lee*,†

†State Key Laboratory of Supramolecular Structure and Materials, College of Chemistry, Jilin University, Changchun 130012, China.

\#UU-KIST Graduate School of Converging Science \& Technology, Korea University, Seoul, Korea.

§Pohang Accelerator Laboratory, Postech, Pohang, Gyeongbuk, Korea.

*Correspondence to: mslee@jlu.edu.cn

\section{Table of Contents}

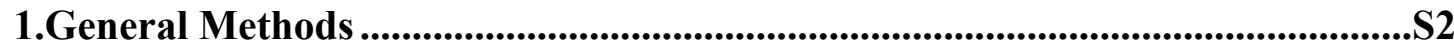

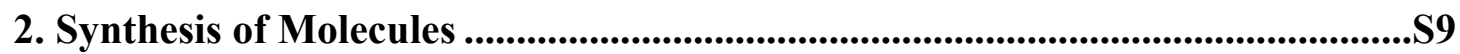

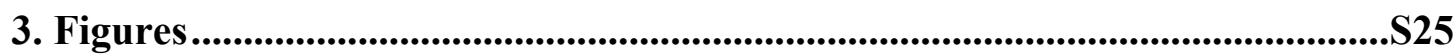

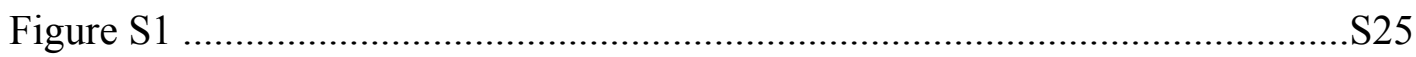

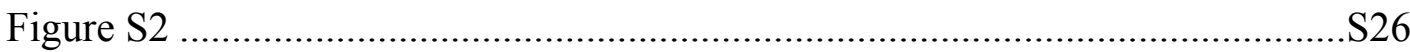

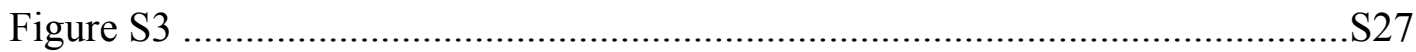

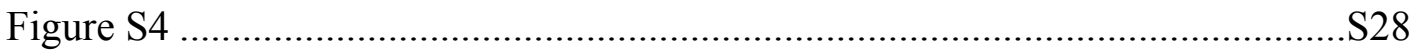

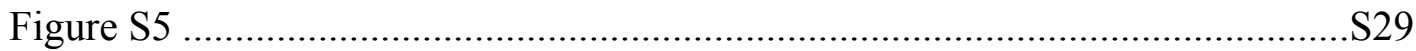

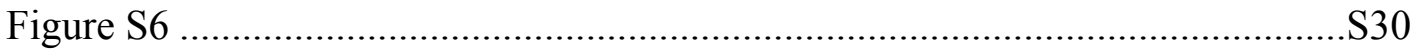

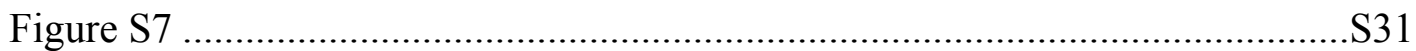

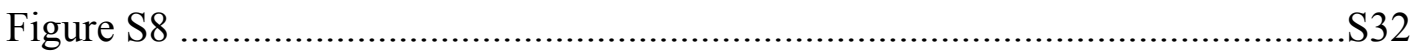

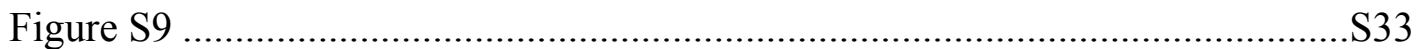

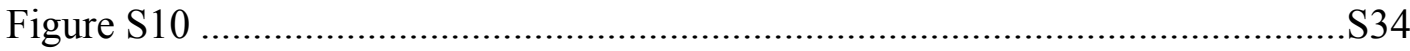

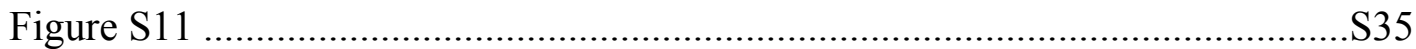

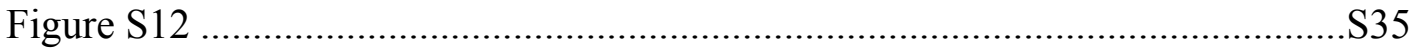

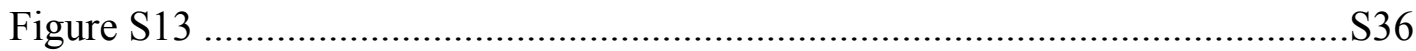

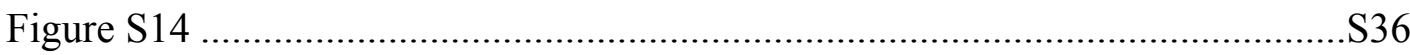




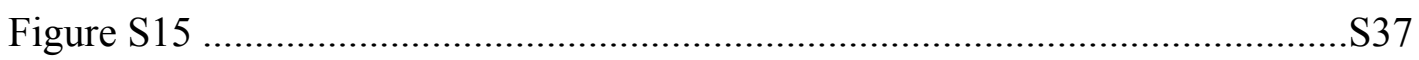

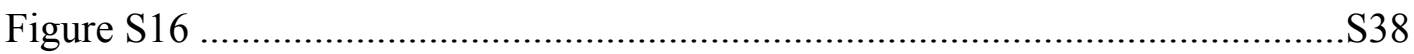

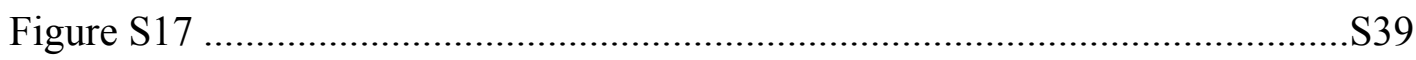

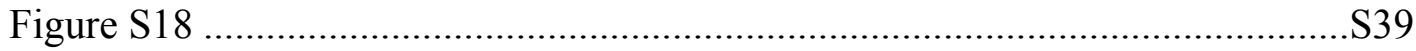

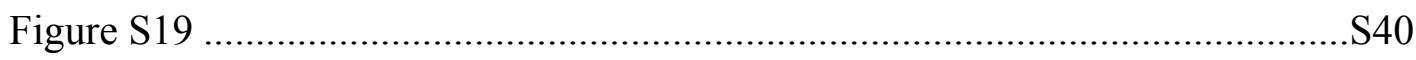

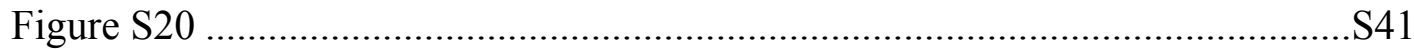

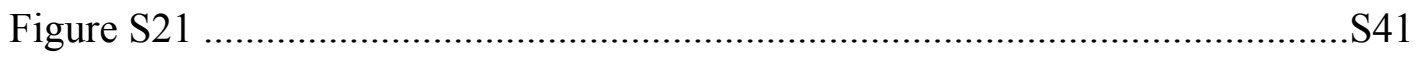

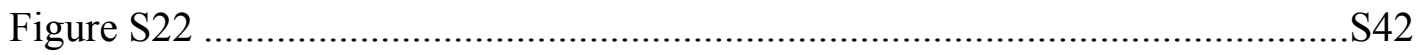

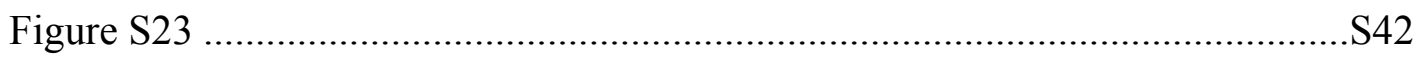

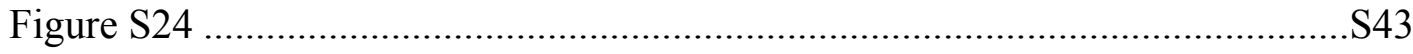

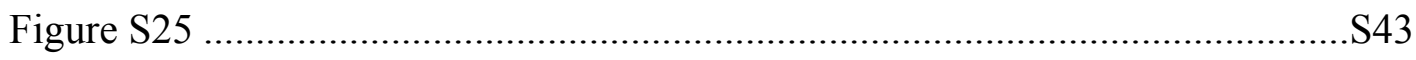

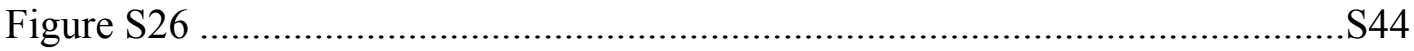

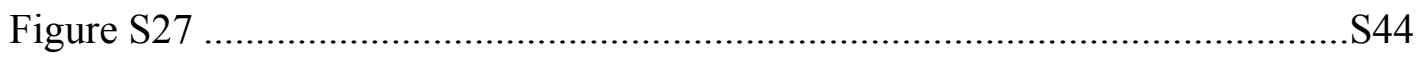

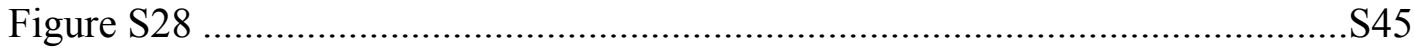

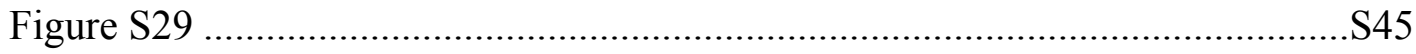

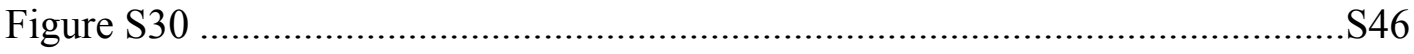

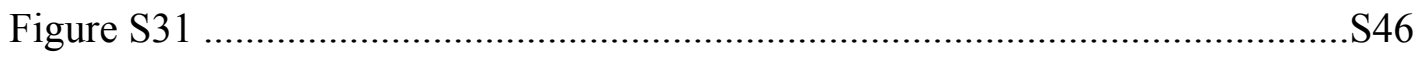

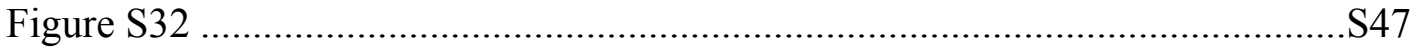

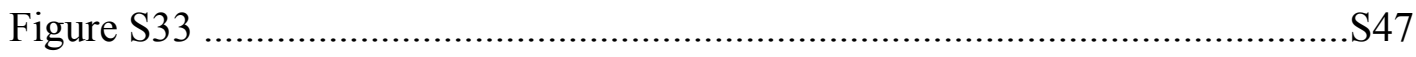

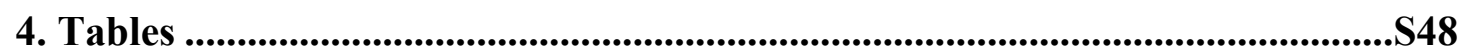

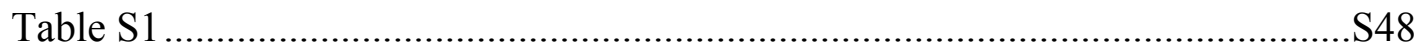

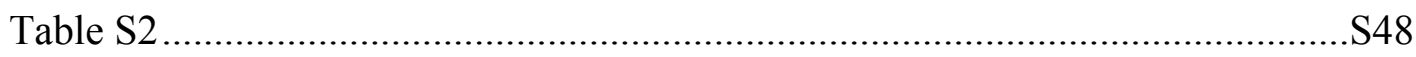

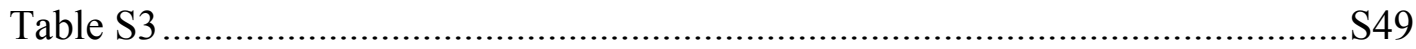

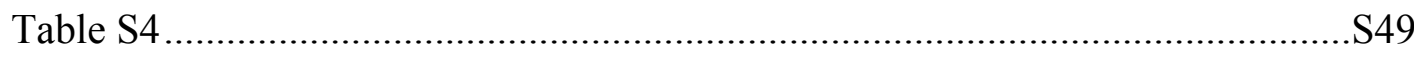

5. Supplementary References....................................................................................S49 


\section{General Methods.}

All reactions were performed in oven-dried glassware under a dry argon atmosphere. Toluene and tetrahydrofuran (THF) were dried by distillation from sodium-benzophenone immediately before use. Acetonitrile $(\mathrm{ACN})$, triethyl amine (TEA) and dichloromethane (DCM) were dried by distillation from $\mathrm{CaH}_{2}$. Acetone was dried by distillation after treatment by $10 \% \mathrm{AgNO}_{3}$ and $1 \mathrm{M} \mathrm{NaOH}$ solution. Distilled water was polished by ion exchange and filtration. Other solvents (anhydrous dimethylformamide (DMF), etc.) and organic reagents were purchased from commercial vendors and used without further purification unless otherwise mentioned. The reactions were monitored by thin-layer chromatography (TLC; Merck, silica gel $60 \mathrm{~F}_{254} 0.25 \mathrm{~mm}$ ) with visualization under ultraviolet light (254 and $365 \mathrm{~nm}$ ) or treating the plates with iodine and $\mathrm{KMnO}_{4}$. The products were purified by flash column chromatography on silica gel (230-400 mesh). ${ }^{1} \mathrm{H}$ NMR and ${ }^{13} \mathrm{C}$ NMR spectra were obtained on Bruker AVANCE III 500 MHz or AVANCE III 600 MHz FT-NMR spectrometers. All compounds were subjected to ${ }^{1} \mathrm{H}$ NMR analysis to confirm $\geq 98 \%$ sample purity. Chemical shifts were reported in ppm relative to the residual solvent peak $\left(\mathrm{CDCl}_{3}:{ }^{1} \mathrm{H}, 7.26 ;{ }^{13} \mathrm{C}, 77.23\right)$ or tetramethylsilane (TMS) peak. Multiplicity was indicated as follows: $\mathrm{s}$ (singlet), $\mathrm{d}$ (doublet), $\mathrm{t}$ (triplet), $\mathrm{m}$ (multiplet), dd (doublet of doublets). All coupling constants ( $\mathrm{J})$ are reported in Hertz (Hz). Matrix-Assisted Laser Desorption/ Ionization Time of Flight Mass Spectrometry (MALDI-TOF-MS) was $\begin{array}{llllll}\text { performed } & \text { on } & \text { a } & \text { Bruker } & \text { Microflex } & \text { TOF }\end{array}$ trans-2-[3-(4-tert-butylphenyl)-2-methyl-2-2-propenylidene]malononitrile (DCTB) as a matrix. Analytic and preparatory high-performance liquid chromatography (HPLC) was performed for further purification of the final desired molecules by using Prominence LC-20AP (SHIMADZU) and YMC C4 reverse-phase column (250 x 4.6 mm I.D. S-5 $\mu \mathrm{m}, 12 \mathrm{~nm} ; 250 \times 20.0 \mathrm{~mm}$ I.D. S-5 $\mu \mathrm{m}, 12 \mathrm{~nm})$. The fluorescence spectra were obtained from a Hitachi F-7000 Fluorescence Spectrophotometer. X-ray scattering measurements were performed in transmission mode with synchrotron radiation at the $3 \mathrm{C} \mathrm{X}$-ray beamline at Pohang Accelerator Laboratory, Korea. X-ray photoelectron spectroscopy (XPS) was performed on an ESCALAB250 X-ray 
photoelectron spectrometer with a monochromatic X-ray source (Al Ka). Dynamic Light Scattering (DLS) measurements were performed with a Malvern type Zetasizer Nano ZS instrument at ambient temperature using a quartz cell of $10 \mathrm{~mm}$ optical path length. The Fluorescence optical microscopy (FOM) image was observed with a Nikon Eclipse TE2000-U inverted fluorescence microscope equipped with a DXM1200C digital camera.

TEM experiments. To investigate the self-assembled structures, a drop of each sample solution was placed on a carbon-coated copper grid (Carbon Type B (15-25 nm) on 200 mesh, with Formvar; Ted Pella, Inc.) and the solvent was allowed to evaporate under ambient conditions. These samples were stained by depositing a drop of uranyl acetate aqueous solution (0.2-0.4 wt \%) onto the surface of the sample-loaded grid. The dried specimen was observed using an FEI Talos-F200C instrument operated at $200 \mathrm{kV}$. The cryogenic transmission electron microscopy (cryo-TEM) experiments were performed with a thin film of sample solution of amphiphiles $(3 \mu \mathrm{l})$ transferred to a lacey supported grid at room temperature (Lacey Formvar/Carbon, 200mesh, $\mathrm{Cu}$; Ted Pella, Inc). The thin films were prepared under controlled temperature and humidity conditions (100\%) within a custom-built environmental chamber to prevent evaporation of solvents from sample solution. The excess liquid was blotted with filter paper for 2 seconds, and the thin films were rapidly vitrified by plunging them into liquid ethane (cooled by liquid nitrogen) at its freezing point. The grid was transferred, on a Gatan 626 cryoholder, using a cryo-transfer device and transferred to the FEI Talos-F200C TEM. Direct imaging was carried out at a temperature of approximately $-175{ }^{\circ} \mathrm{C}$ and with a $200 \mathrm{kV}$ accelerating voltage and the images were acquired with a Ceta 16M CMOS camera.

AFM experiments. The sample films on mica surface were prepared from evaporation of sample solutions ${ }^{\mathrm{S} 1}$. The measurements were conducted on a MultiMode 8 AFM with NanoScope V controller, NanoScope software and NanoScope Analysis software (Bruker AXS Corporation, Santa Barbara, CA, USA) in air at ambient temperature (ca. $25^{\circ} \mathrm{C}$ ) in the scanasyst in air mode. 
XRD experiments. To prepare the powder sample, the water-methanol (3/7, v/v) of 1 was treated with freeze-drying. The freeze-dried sample was then transferred to the X-ray holder. To confirm the stability of the porous nanosheet structures after reaction, the solution after reaction and removed precipitate was frozen dry. Then the freeze-dried sample was also transferred to the X-ray holder.

NMR experiments. Interaction between porous nanosheets and reactant was confirmed by ${ }^{1} \mathrm{H}-\mathrm{NMR} . \quad 1 \quad(912 \mu \mathrm{M})$ and $\mathbf{R} 1(50 \mu \mathrm{M})$ were prepared in $\mathrm{D}_{2} \mathrm{O}$-methanol- $d_{4}$ mixture $(3 / 7, \mathrm{v} / \mathrm{v})$ with sonication for $30 \mathrm{~min}$ and stabilized for $8 \mathrm{hr}$, then performed ${ }^{1} \mathrm{H}-\mathrm{NMR}$ experiment on the $600 \mathrm{MHz}$ FT NMR spectrometer. For control experiment, pure R1 without $\mathbf{1}$ was followed the same method.

XPS experiments. To confirm the coordination of $\mathrm{Pd}$ with porous nanosheets from self-assembly of $1(91 \mu \mathrm{M})$ in water-methanol solution $(3 / 7, \mathrm{v} / \mathrm{v})$, the reference solution of 1 and the solution containing Pd-coordinated porous nanosheets after filtration were placed on a silica wafer, respectively. And the solution was evaporated at ambient temperature.

Uptake and titration experiments. The reactant (R1, R2, R3, R4 and R5) (10 $\mu \mathrm{M})$ was dissolved in chloroform solution followed by evaporation of solvent to get film. Then $0.5 \mathrm{ml}$ water-methanol $(3 / 7, \mathrm{v} / \mathrm{v})$ of $1(91 \mu \mathrm{M})$ was added. The sample solutions were sonicated for $30 \mathrm{~min}$ and stabilized for $8 \mathrm{hr}$ at room temperature. For stoichiometric experiments, sample solutions with $1 \mu \mathrm{M}, 2 \mu \mathrm{M}, 3 \mu \mathrm{M}, 4 \mu \mathrm{M}, 5 \mu \mathrm{M}, 6$ $\mu \mathrm{M}$ and $7 \mu \mathrm{M}$ of $\mathbf{R} 1$ based on $91 \mu \mathrm{M}$ of $\mathbf{1}$ in water-methanol $(3 / 7, \mathrm{v} / \mathrm{v})$ were prepared. After $8 \mathrm{hr}$, the samples $(0.2 \mathrm{ml})$ were run through a Sephadex LH-20 column to remove untrapped reactant molecules. The first fraction was collected and concentrated under reduced pressure. Concentrated fraction was monitored by HPLC analysis. The uptake of reactant within the pores was measured with a $\mathrm{C} 4$ column ( $250 \times 4.6 \mathrm{~mm}$ I.D. S-5 $\mu \mathrm{m}, 12 \mathrm{~nm}$ ). For titration experiment of FL spectra, sample solutions with $1 \mu \mathrm{M}, 2 \mu \mathrm{M}, 3 \mu \mathrm{M}, 4 \mu \mathrm{M}, 5 \mu \mathrm{M}, 6 \mu \mathrm{M}$ and $7 \mu \mathrm{M}$ of R1 based on 91 $\mu \mathrm{M}$ of 1 in water-methanol $(3 / 7, v / v)$ were prepared. The sample solutions were sonicated for $30 \mathrm{~min}$ and stabilized for $8 \mathrm{hr}$ at room temperature before using in the 
experiment.

Molecular dynamic simulations. The folded conformation and size of the reactants were simulated with MD using the Desmond module from Schrödinger Suites with the following parameters-force field: OPLS3; solvent: SPC; simulation temperature: 300.0 K; pressure: 1.01325 bar; simulation time: $10 \mathrm{~ns}$.

Macrocyclization in the presence of porous nanosheets of Suzuki-Miyaura coupling reaction. The reaction solution was prepared by $\mathbf{R} 1$ (or $\mathbf{R 2})(10 \mu \mathrm{M})$ dry film with the addition of $1(91 \mu \mathrm{M})$ of water-methanol solution $(3 / 7, \mathrm{v} / \mathrm{v})$. The sample solutions were sonicated for $30 \mathrm{~min}$ and stabilized for $8 \mathrm{hr}$ at room temperature. The untrapped reactant was removed by Sephadex LH-20 column. After concentrated solution to a final concentration of 1 of $91 \mu \mathrm{M}, \mathrm{Pd}(0)\left[\mathrm{P}(\mathrm{t}-\mathrm{Bu})_{3}\right]_{2}(0.05 \mu \mathrm{M}), \mathrm{NaHCO}_{3}$ $(50 \mu \mathrm{M})$ were added. The reaction mixture was stirred for $4 \mathrm{hr}$ at room temperature under $\mathrm{N}_{2}$. The additional experiment with excess amount of $\mathbf{R} \mathbf{1}$ in the presence of porous material of $\mathbf{1}$ also performed. The reaction solution was prepared by R1 (10 $\mu \mathrm{M})$ dry film with the addition of $1(91 \mu \mathrm{M})$ of water-methanol solution $(3 / 7, \mathrm{v} / \mathrm{v})$. The sample solutions were sonicated for $30 \mathrm{~min}$ without the removal of the untrapped R1 and stabilized for $8 \mathrm{hr}$ at room temperature. Then $\operatorname{Pd}(0)\left[\mathrm{P}(\mathrm{t}-\mathrm{Bu})_{3}\right]_{2}(0.1 \mu \mathrm{M})$, $\mathrm{NaHCO}_{3}(100 \mu \mathrm{M})$ were added. The reaction mixture was stirred for $12 \mathrm{hr}$ at room temperature under $\mathrm{N}_{2}$.

Macrocyclization in the porous nanosheet of Knoevenagel condensation reaction. The reaction solution was prepared by $\mathbf{R 5}(10 \mu \mathrm{M})$ dry film with the addition of $\mathbf{1}$ (91 $\mu \mathrm{M})$ of water-methanol solution $(3 / 7, \mathrm{v} / \mathrm{v})$. The sample solutions were sonicated for $30 \mathrm{~min}$ and stabilized for $2 \mathrm{hr}$ at room temperature. The untrapped reactant was removed by Sephadex LH-20 column. After concentrated solution to a final concentration of 1 of $91 \mu \mathrm{M}, \mathrm{KOH}(5.5 \mu \mathrm{M})$ were added. The reaction mixture was stirred for $80 \mathrm{~min}$ at room temperature.

Control experiments for Suzuki-Miyaura coupling reaction without porous nanosheets. The reaction solution was prepared by $\mathbf{R} 1(5 \mu \mathrm{M})$ dry film and 
toluene-EtOH $(2 / 1, \mathrm{v} / \mathrm{v})$ solution. $\mathrm{Pd}(0)\left[\mathrm{P}(\mathrm{t}-\mathrm{Bu})_{3}\right]_{2}(0.05 \mu \mathrm{M})$ and $\mathrm{NaHCO}_{3}(50 \mu \mathrm{M})$ were added, then stirred for $48 \mathrm{hr}$ at $75^{\circ} \mathrm{C}$ under $\mathrm{N}_{2}$.

Control experiments for Knoevenagel condensation reaction without porous nanosheets. The reaction solution was prepared by $\mathbf{R 5}(5 \mu \mathrm{M})$ dry film and $\mathrm{MeOH} / \mathrm{CHCl}_{2}(1 / 1, \mathrm{v} / \mathrm{v})$ solution. $\mathrm{KOH}(5.5 \mu \mathrm{M})$ were added, then stirred for $80 \mathrm{~min}$ at $60^{\circ} \mathrm{C}$.

Selective macrocyclization. The reaction solution was prepared by evaporating a mixture solution of $\mathbf{R} \mathbf{1}(5 \mu \mathrm{M}), \mathbf{R} 3(5 \mu \mathrm{M})$ and $\mathbf{R} \mathbf{4}$, then with the addition of $\mathbf{1}(91 \mu \mathrm{M})$ of water-methanol solution (3/7, v/v). After $30 \mathrm{~min}$ sonication and $8 \mathrm{hr}$ stabilization at room temperature, then $\mathrm{Pd}(0)\left[\mathrm{P}(\mathrm{t}-\mathrm{Bu})_{3}\right]_{2}(0.05 \mu \mathrm{M})$ and $\mathrm{NaHCO}_{3}(50 \mu \mathrm{M})$ were added. The reaction mixture was stirred for $4 \mathrm{hr}$ at room temperature under $\mathrm{N}_{2}$.

Determination of conversion. The conversion was confirmed by HPLC analysis with C4 column (mobile phase: $85 \%$ ACN to $100 \%$ ACN gradient eluent, flow rate, 0.7 $\left.\mathrm{ml} \cdot \mathrm{min}^{-1}, \lambda=254 \mathrm{~nm}\right)$. The qualitative analysis was determined by retention time, which is as follows: $t_{\mathbf{R} 1}=42.5 \mathrm{~min}, \mathrm{t}_{\mathbf{P} 1}=39.6 \mathrm{~min}, \mathrm{t}_{\mathbf{R} 2}=37.5 \mathrm{~min}, \mathrm{t}_{\mathbf{P} 2}=36.3 \mathrm{~min}, \mathrm{t}_{\mathbf{R} 5}=$ $28.5 \mathrm{~min}, \mathrm{t}_{\mathbf{P 5}}=37.2 \mathrm{~min}$. The conversion of selective macrocyclization reaction was analyzed with $\mathrm{C} 4$ column (mobile phase: $100 \% \mathrm{ACN}$, flow rate, $0.7 \mathrm{ml} \cdot \mathrm{min}^{-1}, \lambda=254$ $\mathrm{nm})$. The qualitative analysis was determined by retention time, which is as follows: $t_{\mathbf{R} 1}=19.9 \mathrm{~min}, \mathrm{t}_{\mathbf{R} 3}=7.5 \mathrm{~min}, \mathrm{t}_{\mathbf{R} 4}=39.0 \mathrm{~min}, \mathrm{t}_{\mathbf{P} 1}=17.7 \mathrm{~min}$.

Spontaneous release of cyclized products experiments. The solution was prepared by $\mathbf{R} 1(10 \mu \mathrm{M})$ dry film with the addition of $\mathbf{1}(91 \mu \mathrm{M})$ water-methanol solution (3/7, $\mathrm{v} / \mathrm{v})$. After $30 \mathrm{~min}$ sonication and $8 \mathrm{hr}$ stabilization at room temperature, the sample was run through a Sephadex LH-20 column to remove untrapped reactant molecules. Then the solution was concentrated to a final concentration of 1 of $91 \mu \mathrm{M}$, and separated into two vials. One vial was added $\mathrm{Pd}(0)\left[\mathrm{P}(\mathrm{t}-\mathrm{Bu})_{3}\right]_{2}(0.05 \mu \mathrm{M})$ and $\mathrm{NaHCO}_{3}$ $(50 \mu \mathrm{M})$, the other vial without any treatment. Then two vials were standing for $12 \mathrm{hr}$ at room temperature. Every hour using separated solution run through a Sephadex LH-20 column and collected first fraction to inject HPLC to confirm the amounts of 
substrates in the pore.

Repeated macrocyclization in porous nanosheets. After completion of reaction and $8 \mathrm{hr}$ standing, the precipitated macrocycle product was removed by decanting. Then R1 $(5 \mu \mathrm{M})$ was added into the supernatant solution. After 30 min sonication and $8 \mathrm{hr}$ stabilization at room temperature, $\operatorname{Pd}(0)\left[\mathrm{P}(\mathrm{t}-\mathrm{Bu})_{3}\right]_{2}(0.05 \mu \mathrm{M})$ was added to the solution. Then next cycle reaction was followed by the same procedure above mentioned. 


\section{Synthesis of Molecules.}
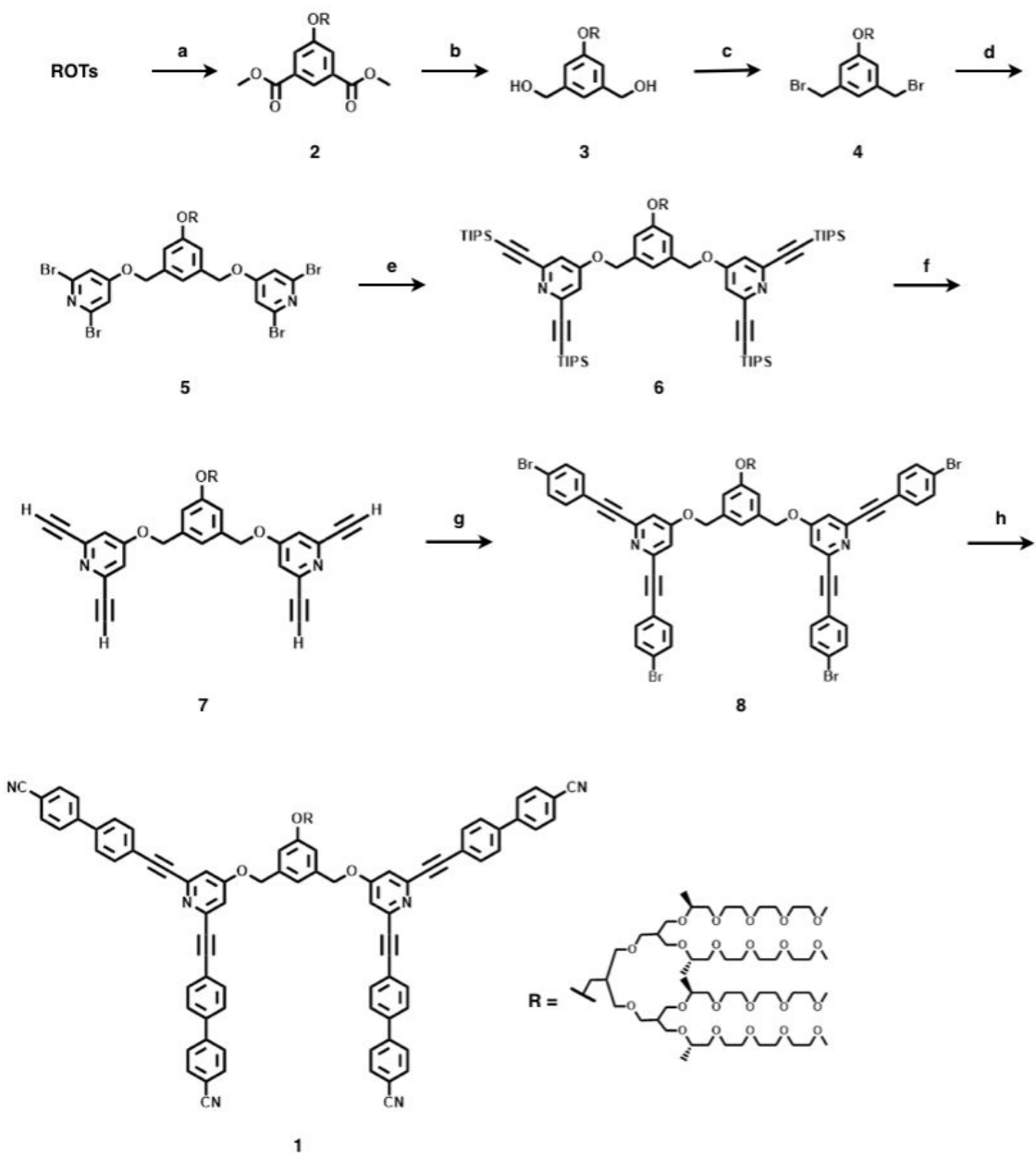

Scheme 1. Synthetic method of molecule 1.

Reagents and conditions: (a) 5-Hydroxyisophthalic acid dimethyl ester, $\mathrm{K}_{2} \mathrm{CO}_{3}$, ACN, reflux, $12 \mathrm{hr}$, yield: 95\%; (b) $\mathrm{LiAlH}_{4}$, THF, $0{ }^{\circ} \mathrm{C}, 2 \mathrm{hr}$, yield: 77\%; (c)

Phosphorus tribromide, DCM, $0{ }^{\circ} \mathrm{C}, 1 \mathrm{hr}$, yield: 55\%; (d) 2,6-Dibromopyridin-4-ol, $\mathrm{K}_{2} \mathrm{CO}_{3}, \mathrm{ACN}$, reflux, $12 \mathrm{hr}$, yield: 94\%; (e) Trimethylsilylacetylene, $\mathrm{PdCl}_{2}\left(\mathrm{PPh}_{3}\right)_{2}$, CuI, TEA, $90{ }^{\circ} \mathrm{C}, 12 \mathrm{hr}$, yield: 78\%; (f) Tetrabutylammonium fluoride, THF, $0{ }^{\circ} \mathrm{C}, 1$ hr, yield: 99\%; (g) 1-Bromo-4-iodobenzene, $\mathrm{Pd}\left(\mathrm{PPh}_{3}\right)_{4}, \mathrm{CuI}$, TEA, $60{ }^{\circ} \mathrm{C}, 12 \mathrm{hr}$, yield: 41\%; (h) 4-Cyanobenzeneboronic acid, $\mathrm{Pd}\left(\mathrm{PPh}_{3}\right)_{4}, 2 \mathrm{M} \mathrm{K}_{2} \mathrm{CO}_{3}(a q)$, EtOH, Toluene, reflux, $12 \mathrm{hr}$, yield: $50 \%$. 
Compound 2. ROTs $^{\mathrm{S} 1}(1.127 \mathrm{~g}, 0.899 \mathrm{mmol})$ and 5-hydroxyisophthalic acid dimethyl ester $(0.227 \mathrm{~g}, 1.080 \mathrm{mmol})$ were dissolved in anhydrous $\mathrm{ACN}(9 \mathrm{ml})$, then $\mathrm{K}_{2} \mathrm{CO}_{3}$ ( $0.625 \mathrm{~g}, 4.498 \mathrm{mmol})$ was added to the mixture. The mixture was refluxed for $12 \mathrm{hr}$ with vigorous stirring under argon. After completion of the reaction as monitored by TLC, the reaction mixture was cooled to room temperature and was extracted with ethyl acetate. The combined organic layers were dried over anhydrous $\mathrm{MgSO}_{4}$. After the filtrate was condensed under the reduced pressure, the crude product was purified by silica gel flash column chromatography using methanol: ethyl acetate (1:10) as an eluent to yield $95 \%$ (1.108 g) of colorless liquid.

${ }^{1} \mathrm{H}$ NMR $\left(500 \mathrm{MHz}, \mathrm{CDCl}_{3}\right) \delta 8.26(\mathrm{~s}, 1 \mathrm{H}), 7.74(\mathrm{~d}, J=1.4 \mathrm{~Hz}, 2 \mathrm{H}), 4.09(\mathrm{~d}, J=5.6$ $\mathrm{Hz}, 2 \mathrm{H}), 3.94(\mathrm{~s}, 6 \mathrm{H}), 3.68-3.31(\mathrm{~m}, 88 \mathrm{H}), 2.37(\mathrm{~m}, 1 \mathrm{H}), 2.06(\mathrm{~m}, 2 \mathrm{H}), 1.09$ (d, $J=$ $6.1 \mathrm{~Hz}, 12 \mathrm{H}) .{ }^{13} \mathrm{C} \mathrm{NMR}\left(126 \mathrm{MHz}, \mathrm{CDCl}_{3}\right) \delta 170.78,159.54,137.36,118.48,113.68$, 75.00, 74.78, 71.93, 70.72, 70.59, 70.51, 69.51, 69.24, 67.41, 66.10, 64.62, 59.01, 40.80, 40.00, 21.01, 17.15. MALDI-TOF mass: $m / z$ calcd. for $\mathrm{C}_{62} \mathrm{H}_{114} \mathrm{NaO}_{27}$ $[\mathrm{M}+\mathrm{Na}]^{+}, 1313.74$; found: 1313.78 .

Compound 3. Compound $2(1.108 \mathrm{~g}, 0.858 \mathrm{mmol})$ was dissolved in anhydrous THF $(12 \mathrm{ml})$, then $\mathrm{LiAlH}_{4}(0.098 \mathrm{~g}, 2.582 \mathrm{mmol})$ was slowly added to solution at $0{ }^{\circ} \mathrm{C}$ ice bath under argon. The mixture was then allowed to gradually turn to room temperature and stirred for $2 \mathrm{hr}$. After completion of the reaction as monitored by TLC, diluted $\mathrm{HCl}$ solution was slowly added to the mixture and the mixture was stirred for $0.5 \mathrm{hr}$. The mixture was extracted with ethyl acetate. The combined organic layers were dried over anhydrous $\mathrm{MgSO}_{4}$. After the filtrate was condensed under the reduced pressure, the crude product was purified by silica gel flash column chromatography using methanol: ethyl acetate (1:10) as an eluent to yield $77 \%(0.820$ g) of colorless liquid.

${ }^{1} \mathrm{H}$ NMR $\left(500 \mathrm{MHz}, \mathrm{CDCl}_{3}\right) \delta 6.90(\mathrm{~s}, 1 \mathrm{H}), 6.87(\mathrm{~s}, 2 \mathrm{H}), 4.63(\mathrm{~s}, 4 \mathrm{H}), 4.06(\mathrm{~d}, J=5.5$ $\mathrm{Hz}, 2 \mathrm{H}), 3.68-3.30(\mathrm{~m}, 88 \mathrm{H}), 2.34(\mathrm{~m}, 1 \mathrm{H}), 2.05(\mathrm{~m}, 2 \mathrm{H}), 1.09$ (d, $J=6.1 \mathrm{~Hz}, 12 \mathrm{H})$. ${ }^{13} \mathrm{C} \mathrm{NMR}\left(126 \mathrm{MHz}, \mathrm{CDCl}_{3}\right) \delta 159.65,143.15,117.58,111.96,75.02,74.79,71.94$, 70.71, 70.59, 70.52, 69.41, 69.25, 67.48, 67.42, 65.90, 64.91, 59.03, 40.81, 39.99, 17.18. MALDI-TOF mass: $m / z$ calcd. for $\mathrm{C}_{60} \mathrm{H}_{114} \mathrm{NaO}_{25}[\mathrm{M}+\mathrm{Na}]^{+}, 1257.75$; found: 1257.76 .

Compound 4. Compound $3(0.820 \mathrm{~g}, 0.663 \mathrm{mmol})$ was dissolved in anhydrous DCM $(7 \mathrm{ml}), \mathrm{PBr}_{3}(227 \mu \mathrm{l}, 2.391 \mathrm{mmol})$ was added dropwise at $0{ }^{\circ} \mathrm{C}$ ice bath. The reaction mixture was stirred for $1 \mathrm{hr}$ under argon. After completion of the reaction as monitored by TLC, the reaction was quenched with $\mathrm{NaHCO}_{3}$. The mixture was extracted with ethyl acetate. The combined organic layers were dried over anhydrous $\mathrm{MgSO}_{4}$. After the filtrate was condensed under the reduced pressure, the crude product was purified by silica gel flash column chromatography using methanol: ethyl acetate $(1: 10)$ as an eluent to yield $55 \%(0.517 \mathrm{~g})$ of pale yellow liquid.

${ }^{1} \mathrm{H} \mathrm{NMR}\left(500 \mathrm{MHz}, \mathrm{CDCl}_{3}\right) \delta 6.98(\mathrm{~s}, 1 \mathrm{H}), 6.86(\mathrm{~s}, 2 \mathrm{H}), 4.42(\mathrm{~s}, 4 \mathrm{H}), 4.01(\mathrm{~d}, J=5.6$ $\mathrm{Hz}, 2 \mathrm{H}), 3.67-3.31(\mathrm{~m}, 88 \mathrm{H}), 2.33(\mathrm{~m}, 1 \mathrm{H}), 2.06(\mathrm{~m}, 2 \mathrm{H}), 1.09(\mathrm{~d}, J=6.2 \mathrm{~Hz}, 12 \mathrm{H})$. ${ }^{13} \mathrm{C}$ NMR $\left(126 \mathrm{MHz}, \mathrm{CDCl}_{3}\right) \delta 159.46,139.55,121.71,115.15,74.96,74.78,71.89$, 
70.70, 70.56, 70.46, 69.68, 69.50, 69.16, 67.34, 66.23, 58.99, 40.74, 39.92, 32.87, 17.07. MALDI-TOF mass: $m / z$ calcd. for $\mathrm{C}_{60} \mathrm{H}_{112} \mathrm{Br}_{2} \mathrm{NaO}_{23}[\mathrm{M}+\mathrm{Na}]^{+}, 1381.59$; found: 1381.65 .

Compound 5. Compound 4 (0.517 g, $0.380 \mathrm{mmol})$ and 2,6-dibromopyridin-4-ol $(0.207 \mathrm{~g}, 0.819 \mathrm{mmol})$ were dissolved in anhydrous $\mathrm{ACN}(4 \mathrm{ml})$, then $\mathrm{K}_{2} \mathrm{CO}_{3}(0.520$ $\mathrm{g}, 3.762 \mathrm{mmol}$ ) was added to the mixture. The mixture was refluxed for $12 \mathrm{hr}$ under argon. After completion of the reaction as monitored by TLC, the reaction mixture was cooled to room temperature and was extracted with DCM. The combined organic layers were dried over anhydrous $\mathrm{MgSO}_{4}$. After the filtrate was condensed under the reduced pressure, the crude product was purified by silica gel flash column chromatography using methanol: ethyl acetate (1:20) as an eluent to yield $94 \%(0.609$ g) of colorless liquid.

${ }^{1} \mathrm{H}$ NMR (500 MHz, $\left.\mathrm{CDCl}_{3}\right) \delta 7.07(\mathrm{~s}, 4 \mathrm{H}), 6.94(\mathrm{~s}, 1 \mathrm{H}), 6.93(\mathrm{~s}, 2 \mathrm{H}), 5.07$ (s, 4H), $4.04(\mathrm{~d}, J=5.7 \mathrm{~Hz}, 2 \mathrm{H}), 3.66-3.31(\mathrm{~m}, 88 \mathrm{H}), 2.35(\mathrm{~m}, 1 \mathrm{H}), 2.06(\mathrm{~m}, 2 \mathrm{H}), 1.08(\mathrm{~d}, J$ $=6.2 \mathrm{~Hz}, 12 \mathrm{H}) .{ }^{13} \mathrm{C} \mathrm{NMR}\left(126 \mathrm{MHz}, \mathrm{CDCl}_{3}\right) \delta 166.49,159.87,141.15,136.69$, 118.33, 114.05, 113.84, 74.99, 74.75, 70.70, 70.55, 70.46, 69.57, 69.14, 67.34, 66.36, 58.96, 40.77, 39.92, 17.10. MALDI-TOF mass: $m / z$ calcd. for $\mathrm{C}_{70} \mathrm{H}_{116} \mathrm{Br}_{4} \mathrm{~N}_{2} \mathrm{NaO}_{25}$ $[\mathrm{M}+\mathrm{Na}]^{+}, 1723.45$; found: 1723.56 .

Compound 6. Compound $5(0.609 \mathrm{~g}, 0.357 \mathrm{mmol})$ was dissolved in anhydrous TEA (4 ml), $\mathrm{PdCl}_{2}\left(\mathrm{PPh}_{3}\right)_{2}(13 \mathrm{mg}, 0.018 \mathrm{mmol})$ and $\mathrm{CuI}(3 \mathrm{mg}, 0.018 \mathrm{mmol})$ were added to the mixture, and then trimethylsilylacetylene $(1.5 \mathrm{ml}, 7.041 \mathrm{mmol})$ was added. The mixture was refluxed for $12 \mathrm{hr}$ with vigorous stirring under argon. After completion of the reaction as monitored by TLC, the reaction mixture was cooled to room temperature and was extracted with ethyl acetate. The combined organic layers were dried over anhydrous $\mathrm{MgSO}_{4}$. After the filtrate was condensed under the reduced pressure, the crude product was purified by silica gel flash column chromatography using methanol: ethyl acetate $(1: 20)$ as an eluent to yield $78 \%(0.582 \mathrm{~g})$ of colorless liquid.

${ }^{1} \mathrm{H}$ NMR $\left(500 \mathrm{MHz}, \mathrm{CDCl}_{3}\right) \delta 7.04(\mathrm{~s}, 5 \mathrm{H}), 6.96(\mathrm{~s}, 2 \mathrm{H}), 5.08(\mathrm{~s}, 4 \mathrm{H}), 4.03(\mathrm{~d}, J=5.6$ $\mathrm{Hz}, 2 \mathrm{H}), 3.67-3.31(\mathrm{~m}, 88 \mathrm{H}), 2.35(\mathrm{~m}, 1 \mathrm{H}), 2.07(\mathrm{~m}, 2 \mathrm{H}), 1.19-1.07(\mathrm{~m}, 96 \mathrm{H}) .{ }^{13} \mathrm{C}$ NMR $\left(126 \mathrm{MHz}, \mathrm{CDCl}_{3}\right) \delta 164.42,159.81,144.76,137.21,118.93,114.26,113.83$, 105.47, 91.89, 75.02, 74.78, 71.92, 70.73, 70.58, 70.50, 69.86, 69.60, 69.18, 67.37, $66.36,58.99,40.79,40.01,18.65,17.12,11.25$. MALDI-TOF mass: $\mathrm{m} / \mathrm{z}$ calcd. for $\mathrm{C}_{114} \mathrm{H}_{200} \mathrm{~N}_{2} \mathrm{NaO}_{25} \mathrm{Si}_{4}[\mathrm{M}+\mathrm{Na}]^{+}, 2132.34$; found: 2132.58 .

Compound 7. Compound 6 (0.201 g, $0.095 \mathrm{mmol})$ was dissolved in anhydrous THF $(9 \mathrm{ml})$ at $0{ }^{\circ} \mathrm{C}$ ice bath, and tetrabutylammonium fluoride $(0.95 \mathrm{ml}, 0.950 \mathrm{mmol})$ was added to the reaction mixture. The mixture was stirred at $0{ }^{\circ} \mathrm{C}$ for $1 \mathrm{hr}$. After completion of the reaction as monitored by TLC, the reaction was quenched with saturated aqueous $\mathrm{NH}_{4} \mathrm{Cl}$, and the mixture was extracted with ethyl acetate. The combined organic layers were dried over anhydrous $\mathrm{MgSO}_{4}$. After the filtrate was condensed under the reduced pressure, the crude product was purified by silica gel 
flash column chromatography using methanol: ethyl acetate $(1: 10)$ as an eluent to yield $99 \%(0.140 \mathrm{~g})$ of colorless liquid.

${ }^{1} \mathrm{H}$ NMR (500 MHz, $\left.\mathrm{CDCl}_{3}\right) \delta 7.12(\mathrm{~s}, 4 \mathrm{H}), 6.98(\mathrm{~s}, 1 \mathrm{H}), 6.95(\mathrm{~s}, 2 \mathrm{H}), 5.13(\mathrm{~s}, 4 \mathrm{H})$, $4.05(\mathrm{~d}, J=5.7 \mathrm{~Hz}, 2 \mathrm{H}), 3.67-3.31(\mathrm{~m}, 88 \mathrm{H}), 3.29(\mathrm{~s}, 4 \mathrm{H}), 2.35(\mathrm{~m}, 1 \mathrm{H}), 2.06(\mathrm{~m}$, 2H), $1.09(\mathrm{~d}, J=6.1 \mathrm{~Hz}, 12 \mathrm{H}) .{ }^{13} \mathrm{C} \mathrm{NMR}\left(126 \mathrm{MHz}, \mathrm{CDCl}_{3}\right) \delta 164.79,159.87$, 143.82, 137.15, 118.28, 114.28, 113.63, 82.08, 77.63, 75.01, 74.79, 70.73, 70.58, 70.50, 69.92, 69.60, 69.20, 67.37, 66.35, 59.00, 40.80, 39.97, 17.13. MALDI-TOF mass: $m / z$ calcd. for $\mathrm{C}_{78} \mathrm{H}_{120} \mathrm{~N}_{2} \mathrm{NaO}_{25}[\mathrm{M}+\mathrm{Na}]^{+}$, 1507.81; found: 1507.80 .

Compound 8. Compound $7(0.131 \mathrm{~g}, 0.091 \mathrm{mmol})$ was dissolved in anhydrous TEA (2 ml), $\mathrm{Pd}\left(\mathrm{PPh}_{3}\right)_{4}(10.4 \mathrm{mg}, 0.0090 \mathrm{mmol})$ and $\mathrm{CuI}(1.03 \mathrm{mg}, 0.0054 \mathrm{mmol})$ were added to the reaction mixture, and then 1-bromo-4-iodobenzene (498 mg, $1.76 \mathrm{mmol}$ ) was added. The mixture was stirred at $60^{\circ} \mathrm{C}$ for $12 \mathrm{hr}$ under argon. After completion of the reaction as monitored by TLC, the reaction mixture was cooled to room temperature and was extracted with ethyl acetate. The combined organic layers were dried over anhydrous $\mathrm{MgSO}_{4}$. After the filtrate was condensed under the reduced pressure, the crude product was purified by silica gel flash column chromatography using methanol: ethyl acetate (1:30) as an eluent to yield 41\% (0.084 g) of colorless liquid.

${ }^{1} \mathrm{H}$ NMR $\left(500 \mathrm{MHz}, \mathrm{CDCl}_{3}\right) \delta 7.51-7.48(\mathrm{~m}, 8 \mathrm{H}), 7.46-7.43(\mathrm{~m}, 8 \mathrm{H}), 7.12(\mathrm{~s}, 4 \mathrm{H})$, $7.04(\mathrm{~s}, 1 \mathrm{H}), 6.98(\mathrm{~s}, 2 \mathrm{H}), 5.14(\mathrm{~s}, 4 \mathrm{H}), 4.06(\mathrm{~d}, J=5.6 \mathrm{~Hz}, 2 \mathrm{H}), 3.65-3.30(\mathrm{~m}, 88 \mathrm{H})$, $2.36(\mathrm{~m}, 1 \mathrm{H}), 2.06(\mathrm{~m}, 2 \mathrm{H}), 1.08(\mathrm{~d}, J=6.1 \mathrm{~Hz}, 12 \mathrm{H}) .{ }^{13} \mathrm{C} \mathrm{NMR}\left(126 \mathrm{MHz}, \mathrm{CDCl}_{3}\right) \delta$ $164.88,159.88,144.71,137.24,133.47,131.75,123.64,120.91,118.29,113.61$, $113.46,89.20,88.31,75.00,74.79,71.91,70.72,70.57,70.50,69.93,69.64,69.20$, $67.40,66.38,59.01,40.79,40.00,17.13$. MALDI-TOF mass: $\mathrm{m} / z$ calcd. for $\mathrm{C}_{102} \mathrm{H}_{132} \mathrm{Br}_{4} \mathrm{~N}_{2} \mathrm{NaO}_{25}[\mathrm{M}+\mathrm{Na}]^{+}, 2123.58$; found: 2123.67 .

Compound 1. Compound $8(0.075 \mathrm{~g}, 0.033 \mathrm{mmol})$ and 4-cyanobenzeneboronic acid $(0.039 \mathrm{~g}, 0.271 \mathrm{mmol})$ were dissolved in degassed toluene $(0.8 \mathrm{ml}), \mathrm{EtOH}(0.4 \mathrm{ml})$ and $2 \mathrm{M}$ aqueous $\mathrm{K}_{2} \mathrm{CO}_{3}(0.02 \mathrm{ml})$, then $\mathrm{Pd}\left(\mathrm{PPh}_{3}\right)_{4}(0.4 \mathrm{mg}, 0.0003 \mathrm{mmol})$ was added to the mixture. The mixture was refluxed for $12 \mathrm{hr}$ with vigorous stirring under argon. After completion of the reaction as monitored by TLC, the reaction mixture was cooled to room temperature and was extracted with ethyl acetate. The combined organic layers were dried over anhydrous $\mathrm{MgSO}_{4}$. After the filtrate was condensed under the reduced pressure, the crude product was purified by silica gel flash column chromatography using methanol: ethyl acetate $(1: 20)$ as an eluent and further purified by prep-HPLC (C4 column, $\mathrm{ACN}$ : $\left.\mathrm{H}_{2} \mathrm{O}=80: 20\right)$ to provide $50 \%(36 \mathrm{mg})$ yield of white waxy solid.

${ }^{1} \mathrm{H} \mathrm{NMR}\left(500 \mathrm{MHz}, \mathrm{CDCl}_{3}\right) \quad \delta 7.76-7.68(\mathrm{~m}, 24 \mathrm{H}), 7.61-7.59(\mathrm{~m}, 8 \mathrm{H}), 7.19(\mathrm{~s}$, 4H), $7.09(\mathrm{~s}, 1 \mathrm{H}), 7.01(\mathrm{~s}, 2 \mathrm{H}), 5.19(\mathrm{~s}, 4 \mathrm{H}), 4.09(\mathrm{~d}, J=5.5 \mathrm{~Hz}, 2 \mathrm{H}), 3.67-3.31(\mathrm{~m}$, $88 \mathrm{H}), 2.38(\mathrm{~m}, 1 \mathrm{H}), 2.07(\mathrm{~m}, 2 \mathrm{H}), 1.09(\mathrm{~d}, J=6.1 \mathrm{~Hz}, 12 \mathrm{H}) .{ }^{13} \mathrm{C}$ NMR $(126 \mathrm{MHz}$, $\left.\mathrm{CDCl}_{3}\right) \delta 165.17,159.97,144.49,139.80,137.20,132.87,132.72,127.65,127.26$, 122.27, 118.71, 113.79, 113.59, 111.50, 89.24, 75.03, 74.82, 74.79, 71.92, 70.73, $70.60,70.58,70.51,70.13,69.66,69.23,67.39,59.02,40.81,40.03,17.14$. 
MALDI-TOF mass: $m / z$ calcd. for $\mathrm{C}_{130} \mathrm{H}_{148} \mathrm{~N}_{6} \mathrm{NaO}_{25}[\mathrm{M}+\mathrm{Na}]^{+}$, 2216.04; found: $[\mathrm{M}+\mathrm{Na}]^{+}, 2216.05$. 

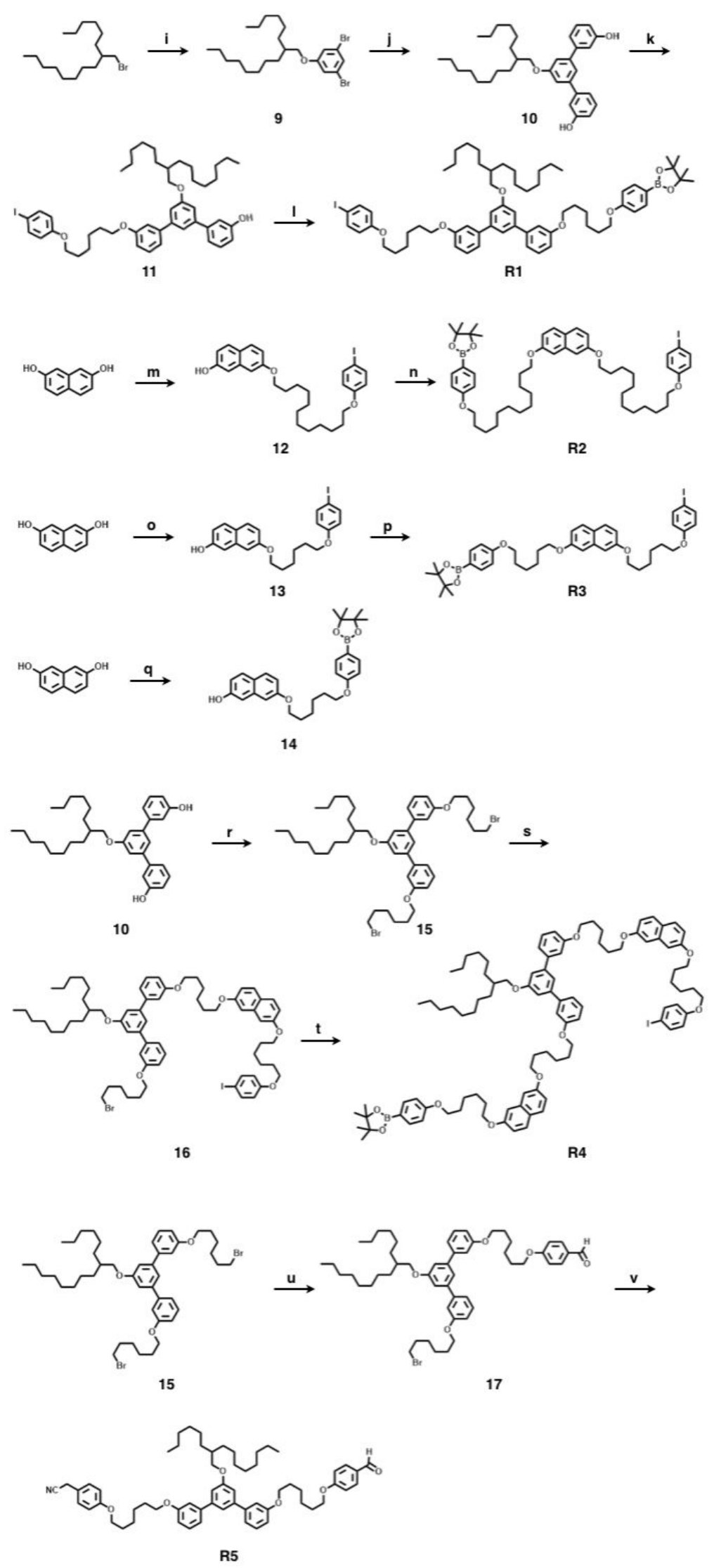

Scheme 2. Synthetic method of molecules R1, R2, R3, R4 and R5. 
Reagents and conditions: (i) 3,5-Dibromophenol, $\mathrm{K}_{2} \mathrm{CO}_{3}, \mathrm{ACN}$, reflux, $12 \mathrm{hr}$, yield: $61 \%$; (j) 3-Hydroxyphenylboronic acid, $\mathrm{Pd}\left(\mathrm{PPh}_{3}\right)_{4}, 2 \mathrm{M} \mathrm{Na}_{2} \mathrm{CO}_{3}(a q), \mathrm{EtOH}$, Toluene, reflux, $12 \mathrm{hr}$, yield: 60\%; (k) 1-(4-Iodophenoxy)-6-bromohexane, $\mathrm{K}_{2} \mathrm{CO}_{3}$, ACN, reflux, $12 \mathrm{hr}$, yield: $20 \%$; (1)

4,4,5,5-Tetramethyl-2-(4-(6-bromohexyloxyphenyl))-1,3,2-dioxaborolane, $\mathrm{K}_{2} \mathrm{CO}_{3}$, ACN, reflux, $12 \mathrm{hr}$, yield: 62\%; (m) 1-((12-Bromo-dodecyl)oxy)-4-iodobenzene, $\mathrm{K}_{2} \mathrm{CO}_{3}$, acetone, reflux, $12 \mathrm{hr}$, yield: $61 \%$; (n)

4,4,5,5-Tetramethyl-2-(4-(12-bromododecyloxyphenyl))-1,3,2-dioxaborolane, $\mathrm{K}_{2} \mathrm{CO}_{3}$, Acetone, reflux, $12 \mathrm{hr}$, yield: 16\%; (o) 1-(4-Iodophenoxy)-6-bromohexane, $\mathrm{K}_{2} \mathrm{CO}_{3}$, Acetone, reflux, $12 \mathrm{hr}$, yield: 50\%; (p)

4,4,5,5-Tetramethyl-2-(4-(6-bromohexyloxyphenyl))-1,3,2-dioxaborolane, $\mathrm{K}_{2} \mathrm{CO}_{3}$, Acetone, reflux, $12 \mathrm{hr}$, yield: 47\%; (q)

4,4,5,5-Tetramethyl-2-(4-(6-bromohexyloxyphenyl))-1,3,2-dioxaborolane, $\mathrm{K}_{2} \mathrm{CO}_{3}$, Acetone, reflux, $12 \mathrm{hr}$, yield: 63\%; (r) 1,6-Dibromohexane, $\mathrm{K}_{2} \mathrm{CO}_{3}, \mathrm{ACN}$, reflux, 12 hr, yield: 47\%; (s) $\mathrm{Cs}_{2} \mathrm{CO}_{3}$, DMF, reflux $12 \mathrm{hr}$, yield: 29\%; (t) $\mathrm{Cs}_{2} \mathrm{CO}_{3}, \mathrm{DMF}$, reflux, $12 \mathrm{hr}$, yield: 19\%. (u) 4-Hydroxybenzaldehyde, ACN, reflux, 12h, yield: 53\%; (v) 4-Hydroxybenzyl cyanide, ACN, reflux, 12h, yield: $24 \%$. 
Compound 9. 7-(Bromomethyl)pentadecane (1.0 g, $3.275 \mathrm{mmol})$ and 3,5-dibromophenol $(1.7 \mathrm{~g}, 6.550 \mathrm{mmol})$ were dissolved in anhydrous ACN (40 ml), then $\mathrm{K}_{2} \mathrm{CO}_{3}(2.2 \mathrm{~g}, 15.92 \mathrm{mmol})$ was added to the mixture. The mixture was refluxed for $12 \mathrm{hr}$ with vigorous stirring under argon. After completion of the reaction as monitored by TLC, the reaction mixture was cooled to room temperature and was extracted with ethyl acetate. The combined organic layers were dried over anhydrous $\mathrm{MgSO}_{4}$. After the filtrate was condensed under the reduced pressure, the crude product was purified by silica gel flash column chromatography using hexane as an eluent to yield $38 \%(0.590 \mathrm{~g})$ of colorless liquid.

${ }^{1} \mathrm{H}$ NMR $\left(500 \mathrm{MHz}, \mathrm{CDCl}_{3}\right) \delta 7.22(\mathrm{t}, J=1.7 \mathrm{~Hz}, 1 \mathrm{H}), 6.99(\mathrm{~d}, J=1.7 \mathrm{~Hz}, 2 \mathrm{H}), 3.79$ $(\mathrm{d}, J=5.6 \mathrm{~Hz}, 2 \mathrm{H}), 1.74-1.72(\mathrm{~m}, 1 \mathrm{H}), 1.43-1.26(\mathrm{~m}, 24 \mathrm{H}), 0.88-0.86(\mathrm{~m}, 6 \mathrm{H})$. ${ }^{13} \mathrm{C} \mathrm{NMR}\left(126 \mathrm{MHz}, \mathrm{CDCl}_{3}\right) \delta 160.59,126.07,123.05,116.92,71.42,37.85,31.94$, $31.86,31.29,30.00,29.67,29.59,29.35,26.82,22.71,14.14$. MS (EI) $m / z(\%$ base peak) $475\left(\mathrm{M}^{+}, 21\right), 251(67), 224(100), 138(72)$.

Compound 10. Compound $9(0.590 \mathrm{~g}, 1.239 \mathrm{mmol})$ and 3-hydroxyphenylboronic acid $(0.427 \mathrm{~g}, 3.096 \mathrm{mmol})$ were dissolved in degassed toluene $(12 \mathrm{ml})$, EtOH (6 ml) and $2 \mathrm{M}$ aqueous $\mathrm{Na}_{2} \mathrm{CO}_{3}(3 \mathrm{ml})$, then $\mathrm{Pd}\left(\mathrm{PPh}_{3}\right)_{4}(14 \mathrm{mg}, 0.0123 \mathrm{mmol})$ was added to the mixture. The mixture was refluxed for $12 \mathrm{hr}$ with vigorous stirring under argon. After completion of the reaction as monitored by TLC, the reaction mixture was cooled to room temperature and was extracted with DCM. The combined organic layers were dried over anhydrous $\mathrm{MgSO}_{4}$. After the filtrate was condensed under the reduced pressure, the crude product was purified by silica gel flash column chromatography using ethyl acetate: hexane (1:5) as an eluent to yield 60\% (0.369 g) of pale yellow liquid.

${ }^{1} \mathrm{H}$ NMR (500 MHz, $\left.\mathrm{CDCl}_{3}\right) \delta 7.35-7.29(\mathrm{~m}, 3 \mathrm{H}), 7.23-7.20(\mathrm{~m}, 2 \mathrm{H}), 7.12-7.08$ $(\mathrm{m}, 4 \mathrm{H}), 6.84(\mathrm{dd}, J=8.0,2.5 \mathrm{~Hz}, 2 \mathrm{H}), 3.93(\mathrm{~d}, J=5.6 \mathrm{~Hz}, 2 \mathrm{H}), 1.84-1.82(\mathrm{~m}, 1 \mathrm{H})$, $1.51-1.27(\mathrm{~m}, 24 \mathrm{H}), 0.88-0.86(\mathrm{~m}, 6 \mathrm{H}) .{ }^{13} \mathrm{C} \mathrm{NMR}\left(126 \mathrm{MHz}, \mathrm{CDCl}_{3}\right) \delta 160.02$, $155.99,142.88,142.57,129.95,119.74,118.49,114.49,114.28,112.56,71.18,38.09$, $31.94,31.90,31.45,30.07,29.74,29.63,29.38,26.89,22.71,14.14$. MS (EI) $m / z(\%$ base peak) $502\left(\mathrm{M}^{+}, 8\right), 278(100), 250(12)$.

Compound 11. Compound 10 (0.263 g, $0.523 \mathrm{mmol})$ and 1-(4-iodophenoxy)-6-bromohexane $\mathrm{S}^{\mathrm{S} 2}(121 \mathrm{mg}, 0.263 \mathrm{mmol})$ were dissolved in anhydrous $\mathrm{ACN}(5 \mathrm{ml})$, then $\mathrm{K}_{2} \mathrm{CO}_{3}(359 \mathrm{mg}, 2.604 \mathrm{mmol})$ was added to the mixture. The mixture was refluxed for $12 \mathrm{hr}$ with vigorous stirring under argon. After completion of the reaction as monitored by TLC, the reaction mixture was cooled to room temperature and was extracted with DCM. The combined organic layers were dried over anhydrous $\mathrm{MgSO}_{4}$. After the filtrate was condensed under the reduced pressure, the crude product was purified by silica gel flash column chromatography using ethyl acetate: hexane (1:20) as an eluent to yield $20 \%(82 \mathrm{mg})$ of colorless liquid.

${ }^{1} \mathrm{H}$ NMR $\left(500 \mathrm{MHz}, \mathrm{CDCl}_{3}\right) \delta 7.55-7.51(\mathrm{~m}, 2 \mathrm{H}), 7.36-7.29(\mathrm{~m}, 3 \mathrm{H}), 7.21(\mathrm{dd}, J=$ $7.7,1.2 \mathrm{~Hz}, 2 \mathrm{H}), 7.16(\mathrm{t}, J=2.1 \mathrm{~Hz}, 1 \mathrm{H}), 7.12-7.08(\mathrm{~m}, 3 \mathrm{H}), 6.90(\mathrm{dd}, J=8.1,2.6$ 
$\mathrm{Hz}, 1 \mathrm{H}), 6.85-6.82(\mathrm{~m}, 1 \mathrm{H}), 6.68-6.65(\mathrm{~m}, 2 \mathrm{H}), 4.04(\mathrm{t}, J=6.4 \mathrm{~Hz}, 2 \mathrm{H}), 3.94-$ $3.91(\mathrm{~m}, 4 \mathrm{H}), 1.86-1.83(\mathrm{~m}, 5 \mathrm{H}), 1.51-1.26(\mathrm{~m}, 28 \mathrm{H}), 0.88-0.86(\mathrm{~m}, 6 \mathrm{H}) .{ }^{13} \mathrm{C}$ NMR $\left(126 \mathrm{MHz}, \mathrm{CDCl}_{3}\right) \delta 160.07,159.42,158.94,155.96,142.97,142.92,142.67$, $142.56,138.18,129.96,129.75,119.81,119.71,118.54,116.96,114.45,114.28$, $113.70,113.44,112.72,112.41,82.53,71.15,67.98,67.92,38.12,31.96,31.93$, 31.55, 31.48, 30.19, 30.10, 29.75, 29.66, 29.40, 29.30, 29.13, 26.92, 25.93, 25.87, 22.74, 14.18. MS (EI) $m / z$ (\% base peak) $804\left(\mathrm{M}^{+}, 18\right) 580(40), 453$ (100), 278 (71).

Compound R1. Compound 11 (81 $\mathrm{mg}, 0.099 \mathrm{mmol})$ and

4,4,5,5-tetramethyl-2-(4-(6-bromohexyloxyphenyl))-1,3,2-dioxaborolane ${ }^{\mathrm{S} 3}$ (42 mg, $0.115 \mathrm{mmol})$ were dissolved in anhydrous $\mathrm{ACN}(1 \mathrm{ml})$, then $\mathrm{K}_{2} \mathrm{CO}_{3}(138 \mathrm{mg}, 1.00$ mmol) was added to the mixture. The mixture was refluxed for $12 \mathrm{hr}$ with vigorous stirring under argon. After completion of the reaction as monitored by TLC, the reaction mixture was cooled to room temperature and was extracted with DCM. The combined organic layers were dried over anhydrous $\mathrm{MgSO}_{4}$. After the filtrate was condensed under the reduced pressure, the crude product was purified by silica gel flash column chromatography using ethyl acetate: hexane (1:30) as an eluent to yield $62 \%(69 \mathrm{mg})$ of colorless liquid.

${ }^{1} \mathrm{H}$ NMR $\left(500 \mathrm{MHz}, \mathrm{CDCl}_{3}\right) \delta 7.75-7.71(\mathrm{~m}, 2 \mathrm{H}), 7.55-7.51(\mathrm{~m}, 2 \mathrm{H}), 7.37-7.32$ (m, 3H), $7.21(\mathrm{~d}, J=7.6 \mathrm{~Hz}, 2 \mathrm{H}), 7.16-7.12(\mathrm{~m}, 2 \mathrm{H}), 7.09$ (d, $J=1.5 \mathrm{~Hz}, 2 \mathrm{H}), 6.89$ $-6.87(\mathrm{~m}, 4 \mathrm{H}), 6.68-6.64(\mathrm{~m}, 2 \mathrm{H}), 4.02-3.98(\mathrm{~m}, 6 \mathrm{H}), 3.93(\mathrm{t}, J=6.5 \mathrm{~Hz}, 4 \mathrm{H})$, $1.87-1.80(\mathrm{~m}, 9 \mathrm{H}), 1.31-1.26(\mathrm{~m}, 44 \mathrm{H}), 0.89-0.87(\mathrm{~m}, 6 \mathrm{H}) .{ }^{13} \mathrm{C}$ NMR $(126 \mathrm{MHz}$, $\left.\mathrm{CDCl}_{3}\right) \delta 161.69,160.04,159.43,159.41,158.96,142.93,142.90,142.73,142.71$, $138.15,136.49$, 129.70, 119.69, 119.66, 118.62, 116.93, 113.85, 113.74, 113.70, $113.35,112.57,112.52,83.51,82.45,71.11,67.95,67.93,67.87,67.62,38.09,31.92$, $31.88,31.63,31.46,31.44,30.33,30.05,29.72$, 29.68, 29.61, 29.35, 29.32, 29.28, 29.20, 29.11, 26.90, 26.88, 25.94, 25.91, 25.85, 24.87, 22.70, 14.13. MALDI-TOF mass: $m / z$ calcd. for $\mathrm{C}_{64} \mathrm{H}_{88} \mathrm{BINaO}_{7}[\mathrm{M}+\mathrm{Na}]^{+}, 1129.56$; found: $[\mathrm{M}+\mathrm{Na}]^{+}, 1129.60$.

Compounds 12 and 13. 2,7-dihydroxynaphthalene $(67 \mathrm{mg}, 0.421 \mathrm{mmol})$ and 1-((12-bromododecyl)oxy)-4-iodobenzene $\mathrm{S}^{\mathrm{S} 4}(100 \mathrm{mg}, 0.212 \mathrm{mmol})$ were dissolved in anhydrous acetone $(2 \mathrm{ml})$, then $\mathrm{K}_{2} \mathrm{CO}_{3}(58 \mathrm{mg}, 0.420 \mathrm{mmol})$ was added to the mixture. The mixture was refluxed for $12 \mathrm{hr}$ with vigorous stirring under argon. After completion of the reaction as monitored by TLC, the reaction mixture was cooled to room temperature and was extracted with DCM. The combined organic layers were dried over anhydrous $\mathrm{MgSO}_{4}$. After the filtrate was condensed under the reduced pressure, the crude product was purified by silica gel flash column chromatography using ethyl acetate: hexane (1:50) as an eluent to yield $61 \%(70 \mathrm{mg})$ of a white solid. Compound 12. ${ }^{1} \mathrm{H}$ NMR $\left(500 \mathrm{MHz}, \mathrm{CDCl}_{3}\right) \delta 7.67$ (dd, $\left.J=8.6,3.8 \mathrm{~Hz}, 2 \mathrm{H}\right), 7.59-$ $7.54(\mathrm{~m}, 2 \mathrm{H}), 7.06(\mathrm{~d}, J=2.6 \mathrm{~Hz}, 1 \mathrm{H}), 7.03-6.99(\mathrm{~m}, 2 \mathrm{H}), 6.94(\mathrm{dd}, J=8.8,2.5 \mathrm{~Hz}$, $1 \mathrm{H}), 6.72-6.67(\mathrm{~m}, 2 \mathrm{H}), 4.07(\mathrm{t}, J=6.6 \mathrm{~Hz}, 2 \mathrm{H}), 3.93(\mathrm{t}, J=6.6 \mathrm{~Hz}, 2 \mathrm{H}), 1.89-$ $1.74(\mathrm{~m}, 4 \mathrm{H}), 1.55-1.32(\mathrm{~m}, 16 \mathrm{H})$. MS (EI) $m / z$ (\% base peak) $546\left(\mathrm{M}^{+}, 20\right), 419$ (17), 220 (13), 160 (100), 131 (15). 
Compound 13. ${ }^{1} \mathrm{H}$ NMR $\left(500 \mathrm{MHz}, \mathrm{CDCl}_{3}\right) \delta 7.67(\mathrm{dd}, J=8.7,4.8 \mathrm{~Hz}, 2 \mathrm{H}), 7.58-$ $7.54(\mathrm{~m}, 2 \mathrm{H}), 7.05(\mathrm{~d}, J=2.5 \mathrm{~Hz}, 1 \mathrm{H}), 7.02-6.98(\mathrm{~m}, 2 \mathrm{H}), 6.95(\mathrm{dd}, J=8.8,2.5 \mathrm{~Hz}$, $1 \mathrm{H}), 6.72-6.68(\mathrm{~m}, 2 \mathrm{H}), 4.09$ (t, $J=6.4 \mathrm{~Hz}, 2 \mathrm{H}), 3.96$ (t, $J=6.5 \mathrm{~Hz}, 2 \mathrm{H}), 1.92-$ $1.87(\mathrm{~m}, 4 \mathrm{H}), 1.61-1.56(\mathrm{~m}, 4 \mathrm{H})$. MS (EI) $m / z$ (\% base peak) $462\left(\mathrm{M}^{+}, 56\right), 335(25)$, $176(22), 160$ (100), $131(28)$.

Compounds R2 and R3. Compound 12 (70 mg, $0.128 \mathrm{mmol})$ and 4,4,5,5-tetramethyl-2-(4-(12-bromododecyloxyphenyl))-1,3,2-dioxaborolane ${ }^{\mathrm{S} 5}$ (72 $\mathrm{mg}, 0.153 \mathrm{mmol})$ were dissolved in anhydrous acetone $(1.3 \mathrm{ml})$, then $\mathrm{K}_{2} \mathrm{CO}_{3}(54 \mathrm{mg}$, $0.391 \mathrm{mmol}$ ) was added to the mixture. The mixture was refluxed for $12 \mathrm{hr}$ with vigorous stirring under argon. After completion of the reaction as monitored by TLC, the reaction mixture was cooled to room temperature and was extracted with DCM. The combined organic layers were dried over anhydrous $\mathrm{MgSO}_{4}$. After the filtrate was condensed under the reduced pressure, the crude product was purified by silica gel flash column chromatography using ethyl acetate: hexane $(1: 20)$ as an eluent to yield $16 \%$ (20 mg) of a white solid.

Compound R2. ${ }^{1} \mathrm{H}$ NMR $\left(500 \mathrm{MHz}, \mathrm{CDCl}_{3}\right) \delta 7.75-7.72(\mathrm{~m}, 2 \mathrm{H}), 7.63(\mathrm{~d}, J=8.9$ $\mathrm{Hz}, 2 \mathrm{H}), 7.55-7.51(\mathrm{~m}, 2 \mathrm{H}), 7.02(\mathrm{~d}, J=2.5 \mathrm{~Hz}, 2 \mathrm{H}), 6.98(\mathrm{~m}, 2 \mathrm{H}), 6.90-6.86(\mathrm{~m}$, $2 \mathrm{H}), 6.69-6.64(\mathrm{~m}, 2 \mathrm{H}), 4.05(\mathrm{t}, J=6.5 \mathrm{~Hz}, 4 \mathrm{H}), 3.97(\mathrm{t}, J=6.6 \mathrm{~Hz}, 2 \mathrm{H}), 3.90(\mathrm{t}, J=$ $6.5 \mathrm{~Hz}, 2 \mathrm{H}), 1.86-1.73(\mathrm{~m}, 8 \mathrm{H}), 1.50-1.28(\mathrm{~m}, 44 \mathrm{H}) .{ }^{13} \mathrm{C} \mathrm{NMR}\left(126 \mathrm{MHz}, \mathrm{CDCl}_{3}\right)$ $\delta 161.75,159.01,157.67,138.14,136.47,135.98,129.02,124.13,116.94,116.24$, $116.22,113.86,106.01,83.50,82.38,68.12,67.98,67.97,67.79,29.59,29.57,29.54$, 29.43, 29.40, 29.36, 29.30, 29.23, 29.15, 26.13, 26.03, 25.99, 24.87. MALDI-TOF mass: $m / z$ calcd. for $\mathrm{C}_{52} \mathrm{H}_{75} \mathrm{BIO}_{6}[\mathrm{M}+\mathrm{H}]^{+}, 933.47$; found: $[\mathrm{M}+\mathrm{H}]^{+}, 933.52$.

Compound R3. ${ }^{1} \mathrm{H}$ NMR $\left(500 \mathrm{MHz}, \mathrm{CDCl}_{3}\right) \delta 7.77-7.73(\mathrm{~m}, 2 \mathrm{H}), 7.64(\mathrm{~d}, J=8.9$ $\mathrm{Hz}, 2 \mathrm{H}), 7.56-7.52(\mathrm{~m}, 2 \mathrm{H}), 7.03(\mathrm{~d}, J=2.5 \mathrm{~Hz}, 2 \mathrm{H}), 6.99-6.96(\mathrm{~m}, 2 \mathrm{H}), 6.92-6.88$ (m, 2H), $6.70-6.65(\mathrm{~m}, 2 \mathrm{H}), 4.07(\mathrm{t}, J=6.4 \mathrm{~Hz}, 4 \mathrm{H}), 4.01(\mathrm{t}, J=6.5 \mathrm{~Hz}, 2 \mathrm{H}), 3.94$ $(\mathrm{t}, J=6.4 \mathrm{~Hz}, 2 \mathrm{H}), 1.92-1.80(\mathrm{~m}, 8 \mathrm{H}), 1.58-1.56(\mathrm{~m}, 8 \mathrm{H}), 1.34(\mathrm{~s}, 12 \mathrm{H}) .{ }^{13} \mathrm{C} \mathrm{NMR}$ $\left(126 \mathrm{MHz}, \mathrm{CDCl}_{3}\right) \delta 161.69,158.96,157.63,138.16,136.50,135.95,129.06,124.18$, 116.93, 116.26, 113.86, 106.03, 83.53, 82.46, 67.95, 67.79, 67.74, 67.63, 29.20, 29.10, 25.92, 25.85, 24.87. MALDI-TOF mass: $m / z$ calcd. for $\mathrm{C}_{40} \mathrm{H}_{51} \mathrm{BIO}_{6}[\mathrm{M}+\mathrm{H}]^{+}$, 765.28; found: $[\mathrm{M}+\mathrm{H}]^{+}, 765.39$.

Compound 14. 2,7-Dihydroxynaphthalene (67 mg, $0.426 \mathrm{mmol}$ ) and 4,4,5,5-tetramethyl-2-(4-(6-bromohexyloxyphenyl))-1,3,2-dioxaborolane (80 mg, $0.212 \mathrm{mmol}$ ) were dissolved in anhydrous acetone $(2 \mathrm{ml})$, then $\mathrm{K}_{2} \mathrm{CO}_{3}(144 \mathrm{mg}, 1.042$ mmol) was added to the mixture. The mixture was refluxed for $12 \mathrm{hr}$ with vigorous stirring under argon. After completion of the reaction as monitored by TLC, the reaction mixture was cooled to room temperature and was extracted with DCM. The combined organic layers were dried over anhydrous $\mathrm{MgSO}_{4}$. After the filtrate was condensed under the reduced pressure, the crude product was purified by silica gel flash column chromatography using ethyl acetate: hexane (1:50) as an eluent to yield $63 \%(61 \mathrm{mg})$ of colorless liquid. 
${ }^{1} \mathrm{H}$ NMR (500 MHz, $\left.\mathrm{CDCl}_{3}\right) \delta 7.74(\mathrm{~d}, J=7.9 \mathrm{~Hz}, 2 \mathrm{H}), 7.67-7.62(\mathrm{~m}, 2 \mathrm{H}), 7.02-$ $6.96(\mathrm{~m}, 2 \mathrm{H}), 6.95-6.86(\mathrm{~m}, 4 \mathrm{H}), 4.08-4.04(\mathrm{~m}, 2 \mathrm{H}), 4.01(\mathrm{t}, J=6.3 \mathrm{~Hz}, 2 \mathrm{H}), 1.84$ $-1.82(\mathrm{~m}, 4 \mathrm{H}), 1.60-1.52(\mathrm{~m}, 4 \mathrm{H}), 1.34(\mathrm{~s}, J=2.0 \mathrm{~Hz}, 12 \mathrm{H})$. MS (EI) $m / z(\%$ base peak) $462\left(\mathrm{M}^{+}, 100\right), 335$ (25), 220 (20), 176 (27), 160 (100), 131 (26).

Compound 15. Compound 10 (500 mg, $0.995 \mathrm{mmol})$ and 1,6-dibromohexane (1.5 g, $5.976 \mathrm{mmol})$ were dissolved in anhydrous $\mathrm{ACN}(10 \mathrm{ml})$, then $\mathrm{K}_{2} \mathrm{CO}_{3}(412 \mathrm{mg}, 2.985$ mmol) was added to the mixture. The mixture was refluxed for $12 \mathrm{hr}$ with vigorous stirring under argon. After completion of the reaction as monitored by TLC, the reaction mixture was cooled to room temperature and was extracted with DCM. The combined organic layers were dried over anhydrous $\mathrm{MgSO}_{4}$. After the filtrate was condensed under the reduced pressure, the crude product was purified by silica gel flash column chromatography using ethyl acetate: hexane (1:50) as an eluent to yield $47 \%$ (383 mg) of colorless liquid.

${ }^{1} \mathrm{H}$ NMR $\left(500 \mathrm{MHz}, \mathrm{CDCl}_{3}\right) \delta 7.37-7.33(\mathrm{~m}, 3 \mathrm{H}), 7.21-7.19(\mathrm{~m}, 2 \mathrm{H}), 7.16(\mathrm{t}, J=$ $2.1 \mathrm{~Hz}, 2 \mathrm{H}), 7.10(\mathrm{~d}, J=1.5 \mathrm{~Hz}, 2 \mathrm{H}), 6.90(\mathrm{dd}, J=8.2,2.5 \mathrm{~Hz}, 2 \mathrm{H}), 4.03(\mathrm{t}, J=6.4$ $\mathrm{Hz}, 4 \mathrm{H}), 3.94(\mathrm{~d}, J=5.6 \mathrm{~Hz}, 2 \mathrm{H}), 3.43(\mathrm{t}, J=6.8 \mathrm{~Hz}, 4 \mathrm{H}), 1.94-1.79(\mathrm{~m}, 9 \mathrm{H}), 1.51-$ $1.25(\mathrm{~m}, 32 \mathrm{H}), 0.90-0.85(\mathrm{~m}, 6 \mathrm{H})$. MS (EI) $m / z$ (\% base peak) $826\left(\mathrm{M}^{+}, 12\right), 604$ (100), $442(23), 278(57)$.

Compound 16. Compound 15 (383 mg, $0.462 \mathrm{mmol}$ ) and compound 13 (107 mg, $0.231 \mathrm{mmol})$ were dissolved in anhydrous DMF $(2.3 \mathrm{ml})$, then $\mathrm{Cs}_{2} \mathrm{CO}_{3}(750 \mathrm{mg}$, $2.302 \mathrm{mmol}$ ) was added to the mixture. The mixture was refluxed for $12 \mathrm{hr}$ with vigorous stirring under argon. After completion of the reaction as monitored by TLC, the reaction mixture was cooled to room temperature and removed DMF, then extracted with DCM. The combined organic layers were dried over anhydrous $\mathrm{MgSO}_{4}$. After the filtrate was condensed under the reduced pressure, the crude product was purified by silica gel flash column chromatography using ethyl acetate: hexane (1:80) as an eluent to yield $29 \%(80 \mathrm{mg})$ of colorless liquid.

${ }^{1} \mathrm{H}$ NMR $\left(500 \mathrm{MHz}, \mathrm{CDCl}_{3}\right) \delta 7.63(\mathrm{~d}, J=8.8 \mathrm{~Hz}, 2 \mathrm{H}), 7.53(\mathrm{~d}, J=8.2 \mathrm{~Hz}, 2 \mathrm{H}), 7.38$ $-7.31(\mathrm{~m}, 3 \mathrm{H}), 7.21(\mathrm{~d}, J=7.8 \mathrm{~Hz}, 2 \mathrm{H}), 7.16(\mathrm{~d}, J=6.7 \mathrm{~Hz}, 2 \mathrm{H}), 7.10(\mathrm{~s}, 2 \mathrm{H}), 7.02$ $(\mathrm{s}, 2 \mathrm{H}), 6.97(\mathrm{t}, J=7.5 \mathrm{~Hz}, 2 \mathrm{H}), 6.90(\mathrm{t}, J=7.4 \mathrm{~Hz}, 2 \mathrm{H}), 6.67(\mathrm{~d}, J=8.2 \mathrm{~Hz}, 2 \mathrm{H})$, $4.04(\mathrm{dt}, J=19.7,6.5 \mathrm{~Hz}, 8 \mathrm{H}), 3.94(\mathrm{~d}, J=5.5 \mathrm{~Hz}, 4 \mathrm{H}), 3.42$ (t, $J=6.8 \mathrm{~Hz}, 2 \mathrm{H}), 1.93$ $-1.78(\mathrm{~m}, 14 \mathrm{H}), 1.63-1.18(\mathrm{~m}, 35 \mathrm{H}), 0.89-0.85(\mathrm{~m}, 6 \mathrm{H})$. MALDI-TOF mass: $\mathrm{m} / z$ calcd. for $\mathrm{C}_{68} \mathrm{H}_{91} \mathrm{BrIO}_{6}[\mathrm{M}+\mathrm{H}]^{+}, 1209.50$; found: $[\mathrm{M}+\mathrm{H}]^{+}, 1209.86$.

Compound R4. Compound 16 (80 mg, $0.066 \mathrm{mmol})$ and compound 14 (61 mg, 0.132 mmol) were dissolved in anhydrous DMF $(0.66 \mathrm{ml})$, then $\mathrm{Cs}_{2} \mathrm{CO}_{3}(215 \mathrm{mg}, 0.66$ $\mathrm{mmol}$ ) was added to the mixture. The mixture was refluxed for $12 \mathrm{hr}$ with vigorous stirring under argon. After completion of the reaction as monitored by TLC, the reaction mixture was cooled to room temperature and removed $\mathrm{DMF}$, then extracted with DCM. The combined organic layers were dried over anhydrous $\mathrm{MgSO}_{4}$. After the filtrate was condensed under the reduced pressure, the crude product was purified 
by silica gel flash column chromatography using ethyl acetate: hexane (1:80) as an eluent to yield $19 \%$ (20 mg) of colorless liquid.

${ }^{1} \mathrm{H} \mathrm{NMR}\left(600 \mathrm{MHz}, \mathrm{CDCl}_{3}\right) \delta 7.74(\mathrm{~d}, J=8.0 \mathrm{~Hz}, 2 \mathrm{H}), 7.62(\mathrm{~d}, J=8.8 \mathrm{~Hz}, 4 \mathrm{H}), 7.53$ $(\mathrm{d}, J=8.5 \mathrm{~Hz}, 2 \mathrm{H}), 7.38-7.32(\mathrm{~m}, 3 \mathrm{H}), 7.21(\mathrm{~d}, J=7.7 \mathrm{~Hz}, 2 \mathrm{H}), 7.17(\mathrm{t}, J=1.8 \mathrm{~Hz}$, $2 \mathrm{H}), 7.10(\mathrm{~d}, J=1.3 \mathrm{~Hz}, 2 \mathrm{H}), 7.02(\mathrm{~d}, J=2.8 \mathrm{~Hz}, 4 \mathrm{H}), 6.99-6.95(\mathrm{~m}, 4 \mathrm{H}), 6.92-$ $6.87(\mathrm{~m}, 4 \mathrm{H}), 6.66(\mathrm{~d}, J=8.5 \mathrm{~Hz}, 2 \mathrm{H}), 4.08-4.03(\mathrm{~m}, 12 \mathrm{H}), 4.00(\mathrm{t}, J=6.4 \mathrm{~Hz}, 2 \mathrm{H})$, $3.92(\mathrm{t}, J=6.4 \mathrm{~Hz}, 4 \mathrm{H}), 1.90-1.79(\mathrm{~m}, 17 \mathrm{H}), 1.57-1.22(\mathrm{~m}, 50 \mathrm{H}), 0.88-0.85(\mathrm{~m}$, $6 \mathrm{H}) .{ }^{13} \mathrm{C} \mathrm{NMR}\left(151 \mathrm{MHz}, \mathrm{CDCl}_{3}\right) \delta 160.03,159.44,157.60,142.91,142.71,138.15$, $136.49,135.95,129.69,129.05,124.17,119.67,118.61,116.92,116.23,113.86$, $113.72,113.35,112.54,106.02,71.11,67.94,67.81,67.73,67.62,38.09,31.91$, $31.44,30.05,29.71,29.60,29.33,29.25,29.19,29.10,26.88,26.00,25.97,25.84$, 24.87, 22.69, 14.13. MALDI-TOF mass: $m / z$ calcd. for $\mathrm{C}_{96} \mathrm{H}_{125} \mathrm{BIO}_{11}[\mathrm{M}+\mathrm{H}]^{+}$, 1591.84; found: $[\mathrm{M}+\mathrm{H}]^{+}, 1591.95$.

Compound 17. Compound 15 (1.1 g, $1.2 \mathrm{mmol})$ and 4-hydroxybenzaldehyde (74 $\mathrm{mg}$, $0.6 \mathrm{mmol})$ were dissolved in anhydrous $\mathrm{ACN}(20 \mathrm{ml})$, then $\mathrm{K}_{2} \mathrm{CO}_{3}(829 \mathrm{mg}, 6.0$ mmol) was added to the mixture. The mixture was refluxed for $12 \mathrm{hr}$ with vigorous stirring under argon. After completion of the reaction as monitored by TLC, the reaction mixture was cooled to room temperature and was extracted with DCM. The combined organic layers were dried over anhydrous $\mathrm{MgSO}_{4}$. After the filtrate was condensed under the reduced pressure, the crude product was purified by silica gel flash column chromatography using ethyl acetate: hexane (1:30) as an eluent to yield $53 \%(276 \mathrm{mg})$ of colorless liquid.

${ }^{1} \mathrm{H}$ NMR $\left(500 \mathrm{MHz}, \mathrm{CDCl}_{3}\right) \delta 9.90(\mathrm{~s}, 1 \mathrm{H}), 7.86-7.83(\mathrm{~m}, 2 \mathrm{H}), 7.40-7.35(\mathrm{~m}, 3 \mathrm{H})$, $7.25-7.22(\mathrm{~m}, 2 \mathrm{H}), 7.19$ (q, $J=2.2 \mathrm{~Hz}, 2 \mathrm{H}), 7.12(\mathrm{~d}, J=1.5 \mathrm{~Hz}, 2 \mathrm{H}), 7.03-6.99(\mathrm{~m}$, 2H), $6.92(\mathrm{ddt}, J=8.3,2.3,1.0 \mathrm{~Hz}, 2 \mathrm{H}), 4.10-4.04(\mathrm{~m}, 6 \mathrm{H}), 3.96(\mathrm{~d}, J=5.6 \mathrm{~Hz}, 2 \mathrm{H})$, $3.45(\mathrm{t}, J=6.8 \mathrm{~Hz}, 2 \mathrm{H}), 1.95-1.84(\mathrm{~m}, 9 \mathrm{H}), 1.64-1.26(\mathrm{~m}, 32 \mathrm{H}), 0.92-0.88(\mathrm{~m}$, $6 \mathrm{H})$. MS (EI) $m / z$ (\% base peak) $868\left(\mathrm{M}^{+}, 15\right), 644(89), 565$ (30), 482 (44), 361 (23), 278 (100).

Compound R5. Compound 17 (189 $\mathrm{mg}, 0.22 \mathrm{mmol}$ ) and 4-hydroxybenzyl cyanide (24 mg, $0.18 \mathrm{mmol}$ ) were dissolved in anhydrous $\mathrm{ACN}(2 \mathrm{ml})$, then $\mathrm{K}_{2} \mathrm{CO}_{3}(249 \mathrm{mg}$, $1.8 \mathrm{mmol}$ ) was added to the mixture. The mixture was refluxed for $12 \mathrm{hr}$ with vigorous stirring under argon. After completion of the reaction as monitored by TLC, the reaction mixture was cooled to room temperature and was extracted with DCM. The combined organic layers were dried over anhydrous $\mathrm{MgSO}_{4}$. After the filtrate was condensed under the reduced pressure, the crude product was purified by silica gel flash column chromatography using ethyl acetate: hexane $(1: 6)$ as an eluent to yield $24 \%$ (40 $\mathrm{mg})$ of colorless liquid.

${ }^{1} \mathrm{H}$ NMR $\left(500 \mathrm{MHz}, \mathrm{CDCl}_{3}\right) \delta 9.87(\mathrm{~s}, 1 \mathrm{H}), 7.81(\mathrm{~d}, J=8.7 \mathrm{~Hz}, 2 \mathrm{H}), 7.37-7.33(\mathrm{~m}$, $3 \mathrm{H}), 7.23-7.19(\mathrm{~m}, 4 \mathrm{H}), 7.16(\mathrm{p}, J=1.2 \mathrm{~Hz}, 2 \mathrm{H}), 7.10(\mathrm{~d}, J=1.5 \mathrm{~Hz}, 2 \mathrm{H}), 7.00-$ $6.97(\mathrm{~m}, 2 \mathrm{H}), 6.91-6.86(\mathrm{~m}, 4 \mathrm{H}), 4.08-4.02(\mathrm{~m}, 6 \mathrm{H}), 3.98-3.93(\mathrm{~m}, 4 \mathrm{H}), 3.66(\mathrm{~s}$, $2 \mathrm{H}), 1.90-1.80(\mathrm{~m}, 9 \mathrm{H}), 1.60-1.25(\mathrm{~m}, 32 \mathrm{H}), 0.88(\mathrm{td}, J=6.7,5.3 \mathrm{~Hz}, 6 \mathrm{H}) .{ }^{13} \mathrm{C}$ NMR $\left(126 \mathrm{MHz}, \mathrm{CDCl}_{3}\right) \delta 190.79,164.19,160.05,159.43,159.41,158.86,142.92$, 
$142.89,142.73,142.71,131.99,129.80,129.72,129.04,121.58,119.71,119.68$, $118.59,115.08,114.75,113.75,113.71,113.34,113.32,112.57,112.54,71.12,68.25$, 67.97, 67.89, 67.85, 38.09, 31.92, 31.88, 31.45, 31.44, 30.05, 29.72, 29.61, 29.35, $29.29,29.27,29.17,29.03,26.90,26.88,25.92,25.91,25.88,25.83,22.82,22.70$, 22.69, 14.14. MALDI-TOF mass: $m / z$ calcd. for $\mathrm{C}_{61} \mathrm{H}_{79} \mathrm{NNaO}_{6}[\mathrm{M}+\mathrm{Na}]^{+}, 944.58$; found: $[\mathrm{M}+\mathrm{Na}]^{+}, 944.65$. 

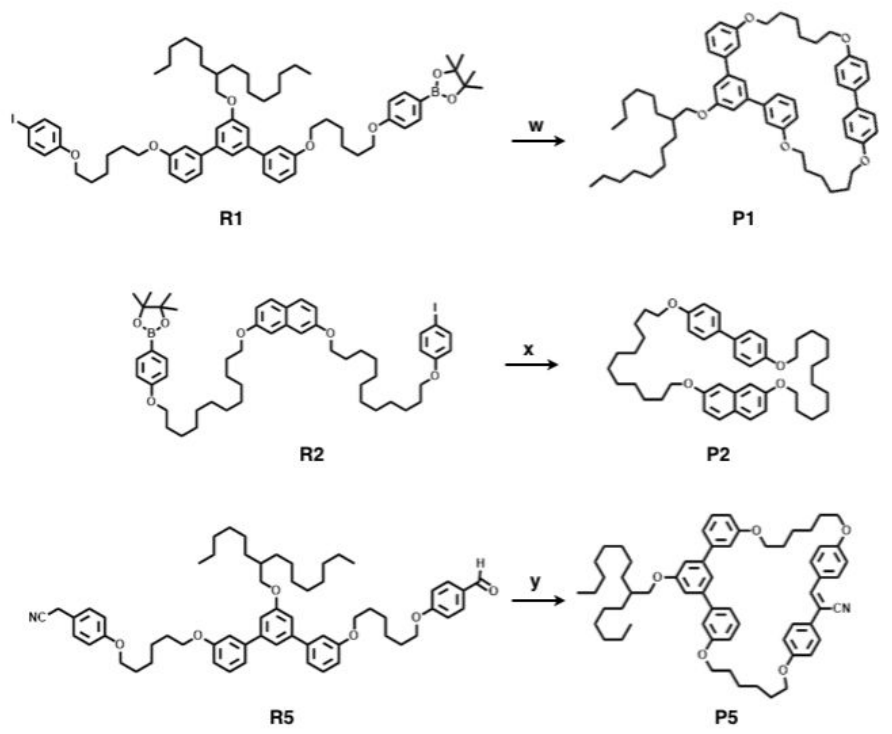

Scheme 3. Synthetic method of molecules P1, P2 and P5.

Reagents and conditions: (w) $\mathrm{Pd}\left(\mathrm{PPh}_{3}\right)_{4}, 2 \mathrm{M} \mathrm{Na}_{2} \mathrm{CO}_{3}$ (aq), EtOH, Toluene, reflux, $48 \mathrm{hr}$, yield: $1 \%$; (x) $\mathrm{Pd}\left(\mathrm{PPh}_{3}\right)_{4}, 2 \mathrm{M} \mathrm{Na}_{2} \mathrm{CO}_{3}(a q)$, EtOH, Toluene, reflux, 48 hr, yield: $1 \%$.(y) $\mathrm{KOH}, \mathrm{MeOH} / \mathrm{CHCl}_{2}(1 / 1, \mathrm{v} / \mathrm{v})$, reflux, $48 \mathrm{hr}$, yield: $5 \%$. 
Compound P1. Compound R1 (1.100 g, $0.994 \mathrm{mmol}$ ) was dissolved in degassed toluene $(99.4 \mathrm{ml})$, EtOH $(49.7 \mathrm{ml})$ and $2 \mathrm{M}$ aqueous $\mathrm{Na}_{2} \mathrm{CO}_{3}(24.9 \mathrm{ml})$, then $\operatorname{Pd}\left(\mathrm{PPh}_{3}\right)_{4}(11.5 \mathrm{mg}, 0.010 \mathrm{mmol})$ were added to the mixture. The mixture was refluxed for 2 days with vigorous stirring under argon. After completion of the reaction as monitored by TLC, the reaction mixture was cooled to room temperature and was extracted with DCM. The combined organic layers were dried over anhydrous $\mathrm{MgSO}_{4}$. After the filtrate was condensed under the reduced pressure, the crude product was purified by silica gel flash column chromatography using ethyl acetate: hexane (1:30) as an eluent to yield $1 \%(10.2 \mathrm{mg})$ of colorless liquid. ${ }^{1} \mathrm{H}$ NMR (500 MHz, $\left.\mathrm{CDCl}_{3}\right) \delta 7.35(\mathrm{~d}, J=1.6 \mathrm{~Hz}, 1 \mathrm{H}), 7.28(\mathrm{t}, J=8.0 \mathrm{~Hz}, 2 \mathrm{H}), 7.25$ $-7.21(\mathrm{~m}, 4 \mathrm{H}), 7.18(\mathrm{dd}, J=7.4,1.6 \mathrm{~Hz}, 2 \mathrm{H}), 7.13(\mathrm{t}, J=2.1 \mathrm{~Hz}, 2 \mathrm{H}), 7.07(\mathrm{~d}, J=$ $1.5 \mathrm{~Hz}, 2 \mathrm{H}), 6.83(\mathrm{dd}, J=8.5,2.9 \mathrm{~Hz}, 6 \mathrm{H}), 4.08(\mathrm{t}, J=6.1 \mathrm{~Hz}, 4 \mathrm{H}), 4.01(\mathrm{t}, J=6.1$ $\mathrm{Hz}, 4 \mathrm{H}), 3.89$ (d, $J=5.6 \mathrm{~Hz}, 2 \mathrm{H}), 1.80-1.76(\mathrm{~m}, 9 \mathrm{H}), 1.34-1.25(\mathrm{~m}, 32 \mathrm{H}), 0.89(\mathrm{~d}$, $J=1.8 \mathrm{~Hz}, 6 \mathrm{H}) .{ }^{13} \mathrm{C} \mathrm{NMR}\left(126 \mathrm{MHz}, \mathrm{CDCl}_{3}\right) \delta 160.00,159.38,157.73,142.79$, $142.68,133.25,129.65,127.50,119.64,118.44,115.22,114.58,112.58,112.39$, 71.06, 67.47, 67.44, 38.08, 31.91, 31.87, 31.63, 31.43, 30.32, 30.06, 29.72, 29.68, 29.60, 29.36, 28.54, 27.96, 26.89, 26.86, 24.86, 24.78, 22.70, 14.13. MALDI-TOF mass: $m / z$ calcd. for $\mathrm{C}_{58} \mathrm{H}_{77} \mathrm{O}_{5}[\mathrm{M}+\mathrm{H}]^{+}, 853.58$; found: 853.59 .

Compound P2 was prepared by the same synthetic method of compound P1. The crude product was purified by silica gel flash column chromatography using ethyl acetate: hexane (1:30) as an eluent to yield $1 \%(7 \mathrm{mg})$ of a white solid.

${ }^{1} \mathrm{H}$ NMR $\left(500 \mathrm{MHz}, \mathrm{CDCl}_{3}\right) \delta 7.60(\mathrm{~d}, J=8.8 \mathrm{~Hz}, 2 \mathrm{H}), 7.44(\mathrm{~d}, J=8.6 \mathrm{~Hz}, 4 \mathrm{H}), 7.00$ $(\mathrm{d}, J=2.1 \mathrm{~Hz}, 2 \mathrm{H}), 6.98-6.91(\mathrm{~m}, 6 \mathrm{H}), 4.02(\mathrm{t}, J=6.4 \mathrm{~Hz}, 8 \mathrm{H}), 1.80$ (dq, $J=20.0$, $6.9 \mathrm{~Hz}, 8 \mathrm{H}), 1.49-1.43(\mathrm{~m}, 8 \mathrm{H}), 1.39-1.28(\mathrm{~m}, 24 \mathrm{H}) .{ }^{13} \mathrm{C} \mathrm{NMR}\left(126 \mathrm{MHz}, \mathrm{CDCl}_{3}\right)$ $\delta 158.11,157.63,135.97,133.29,129.00,127.61,124.12,116.10,115.26,114.99$, 106.11, 67.90, 67.77, 29.27, 29.20, 29.03, 28.99, 28.94, 28.92, 28.63, 28.47, 25.89, 25.50. MALDI-TOF mass: $m / z$ calcd. for $\mathrm{C}_{46} \mathrm{H}_{63} \mathrm{O}_{4}[\mathrm{M}+\mathrm{H}]^{+}, 679.47$; found: 679.50 .

Compound P5. Compound R5 (500 mg, $0.54 \mathrm{mmol}$ ) and KOH (33 mg, $0.594 \mathrm{mmol}$ ) were dissolved in $\mathrm{MeOH}(54 \mathrm{~mL})$. The mixture was refluxed for 2 days with vigorous stirring under argon. The reaction mixture was cooled to room temperature and was extracted with DCM. The combined organic layers were dried over anhydrous $\mathrm{MgSO}_{4}$. After the filtrate was condensed under the reduced pressure, the crude product was purified by silica gel flash column chromatography using ethyl acetate: hexane (1:50) as an eluent to yield 5\% (24.4 mg) of colorless liquid.

${ }^{1} \mathrm{H}$ NMR $\left(500 \mathrm{MHz}, \mathrm{CDCl}_{3}\right) \delta 7.54(\mathrm{~d}, J=8.8 \mathrm{~Hz}, 2 \mathrm{H}), 7.39(\mathrm{~s}, 1 \mathrm{H}), 7.33(\mathrm{~m}, 4 \mathrm{H})$, $7.21(\mathrm{~d}, J=8.0 \mathrm{~Hz}, 2 \mathrm{H}), 7.10-7.08(\mathrm{~m}, 1 \mathrm{H}), 7.03-7.00(\mathrm{~m}, 2 \mathrm{H}), 6.98(\mathrm{~d}, J=1.6 \mathrm{~Hz}$, $1 \mathrm{H}), 6.89(\mathrm{~s}, 1 \mathrm{H}), 6.86-6.80(\mathrm{~m}, 6 \mathrm{H}), 4.14(\mathrm{dt}, J=11.9,5.9 \mathrm{~Hz}, 4 \mathrm{H}), 4.04-3.97(\mathrm{~m}$, $4 \mathrm{H}), 3.87(\mathrm{~d}, J=5.7 \mathrm{~Hz}, 2 \mathrm{H}), 1.88-1.79(\mathrm{~m}, 9 \mathrm{H}), 1.64-1.24(\mathrm{~m}, 32 \mathrm{H}), 0.91-0.87$ $(\mathrm{m}, 6 \mathrm{H}) .{ }^{13} \mathrm{C} \mathrm{NMR}\left(126 \mathrm{MHz}, \mathrm{CDCl}_{3}\right) \delta 160.32,159.99,159.45,159.28,142.57$, $142.39,140.03,130.85,129.72,127.30,126.88,126.54,119.52,118.61,118.25$, 115.24, 115.09, 114.61, 114.41, 71.21, 67.36, 67.16, 66.95, 66.75, 38.09, 31.90, 31.44, 
30.09, 29.74, 29.64, 29.38, 28.49, 27.78, 26.90, 24.41, 24.24, 24.19, 22.71, 14.14 . MALDI-TOF mass: $m / z$ calcd. for $\mathrm{C}_{61} \mathrm{H}_{77} \mathrm{NO}_{5}[\mathrm{M}+\mathrm{H}]^{+}, 904.59$; found: 904.63 . 


\section{Figures}

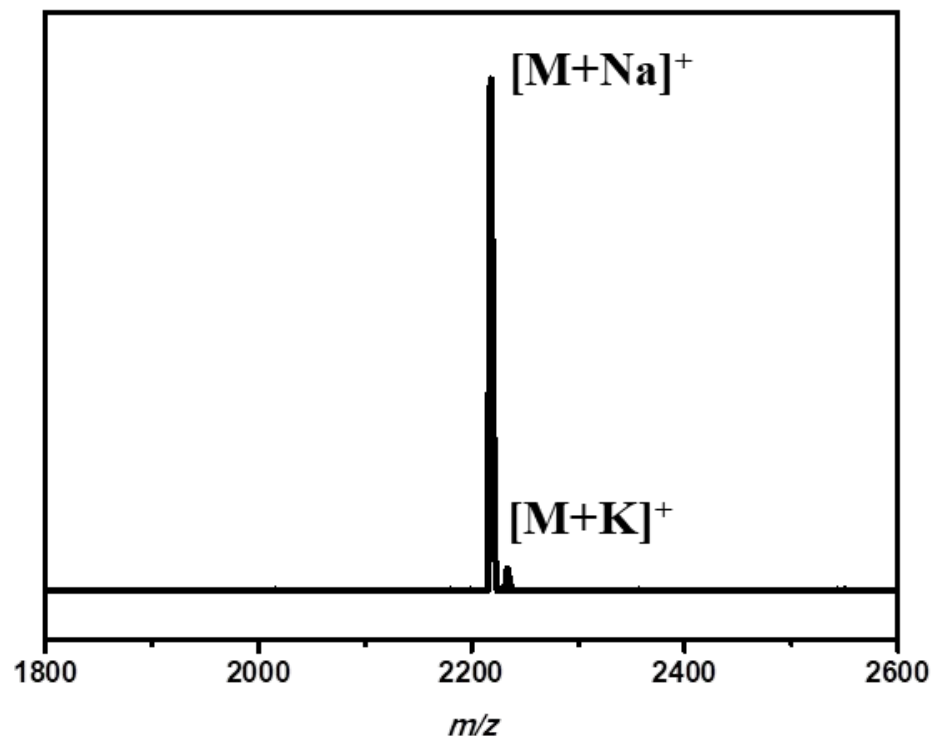

Figure S1. MALDI-TOF mass spectrum of molecule 1. 

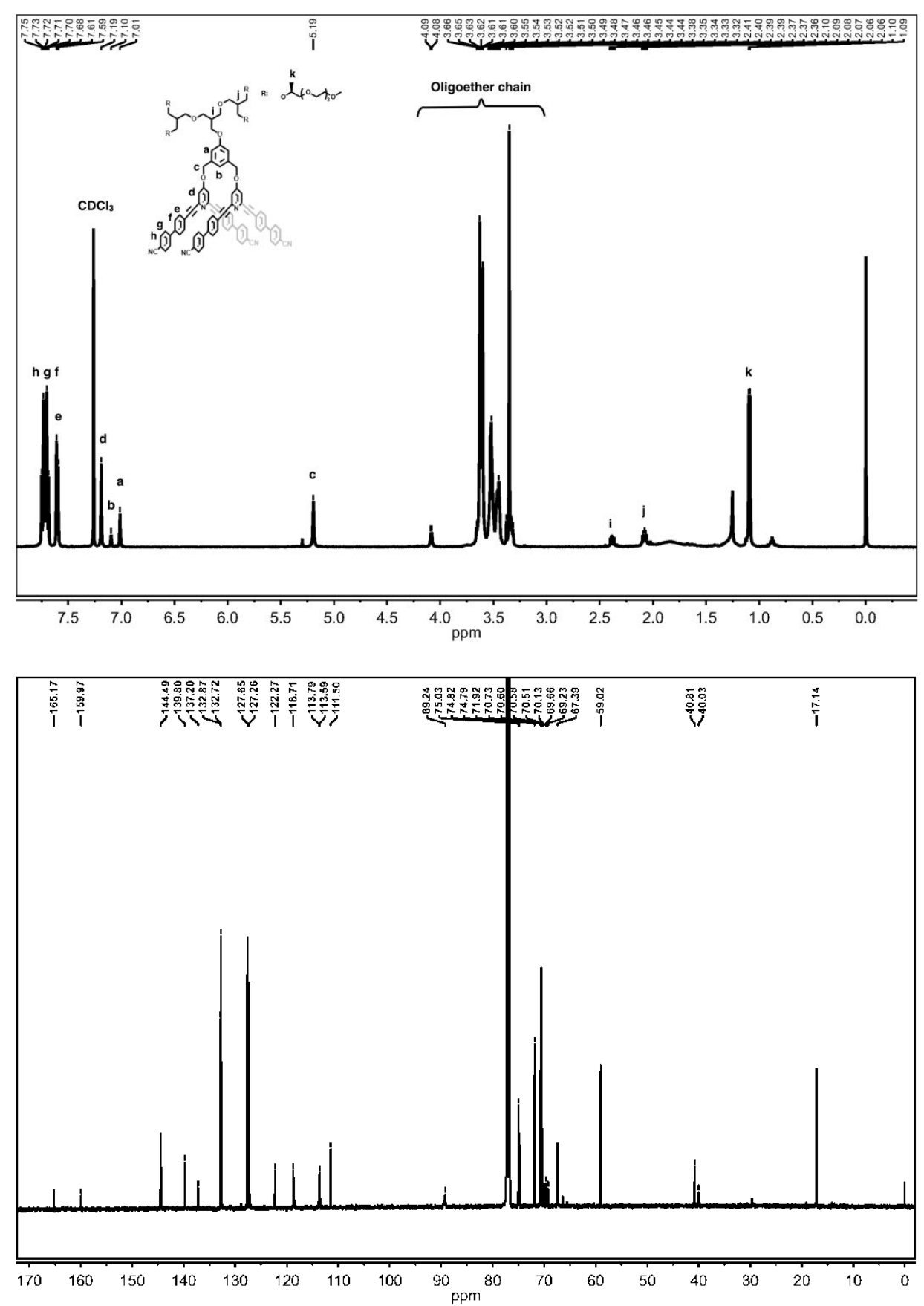

Figure S2. ${ }^{1} \mathrm{H}$ and ${ }^{13} \mathrm{C}$ NMR spectra of 1 in $\mathrm{CDCl}_{3}$. 

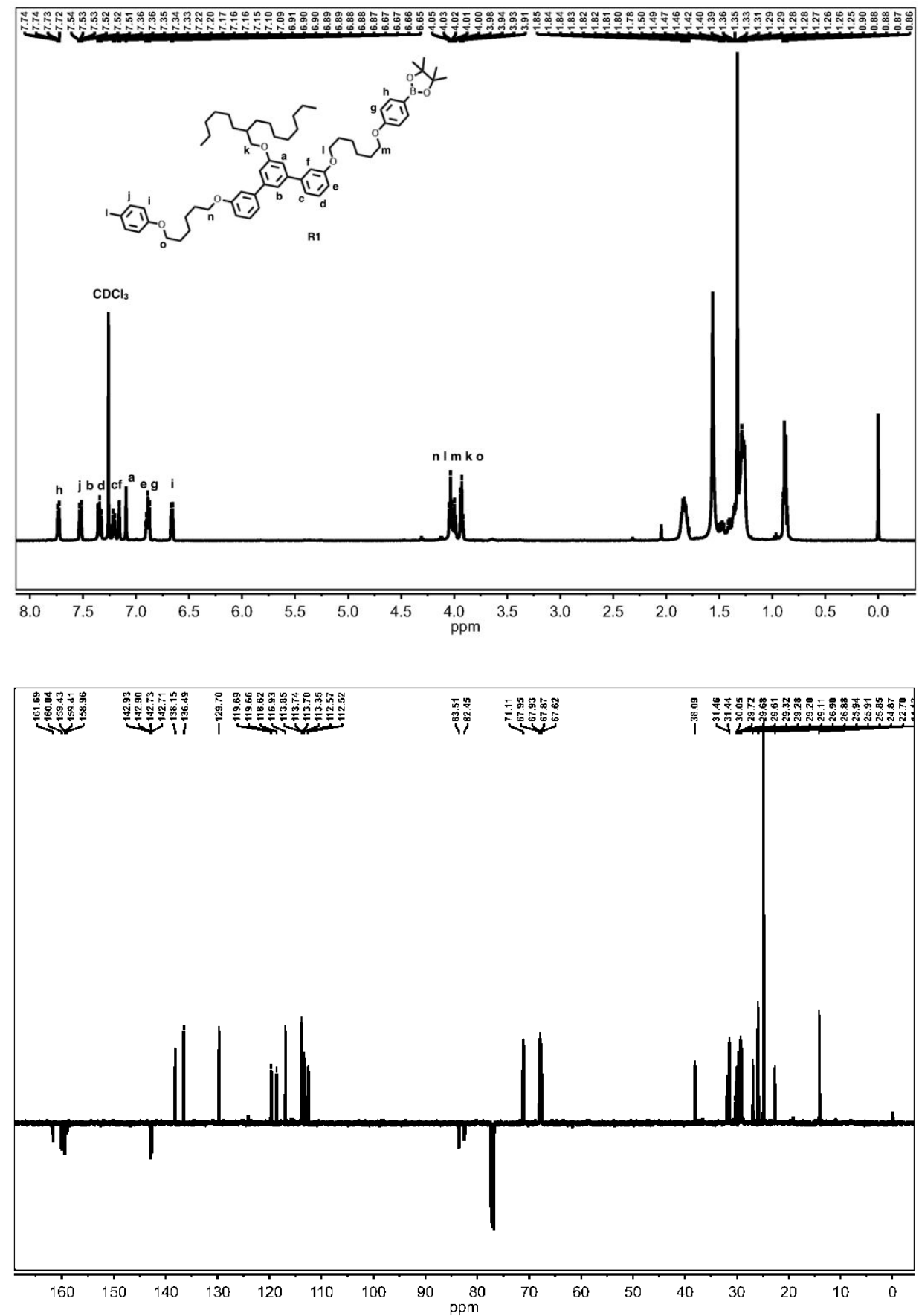

Figure S3. ${ }^{1} \mathrm{H}$ and ${ }^{13} \mathrm{C}$ NMR (DEPTQ) spectra of $\mathbf{R} 1$ in $\mathrm{CDCl}_{3}$. In DEPTQ spectrum, solvent and quaternary carbon showed negative peaks. 

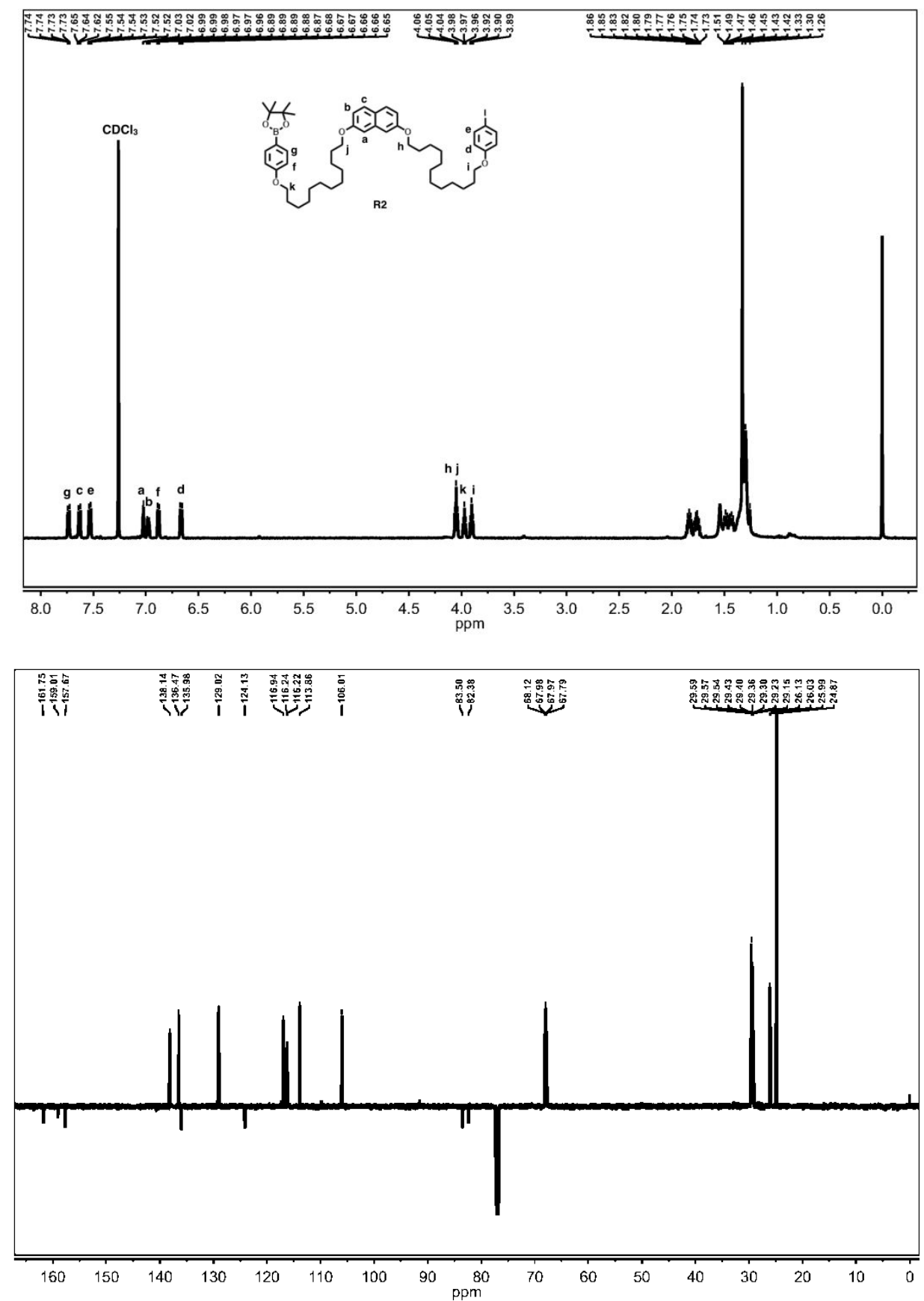

Figure S4. ${ }^{1} \mathrm{H}$ and ${ }^{13} \mathrm{C}$ NMR (DEPTQ) spectra of $\mathbf{R 2}$ in $\mathrm{CDCl}_{3}$. 

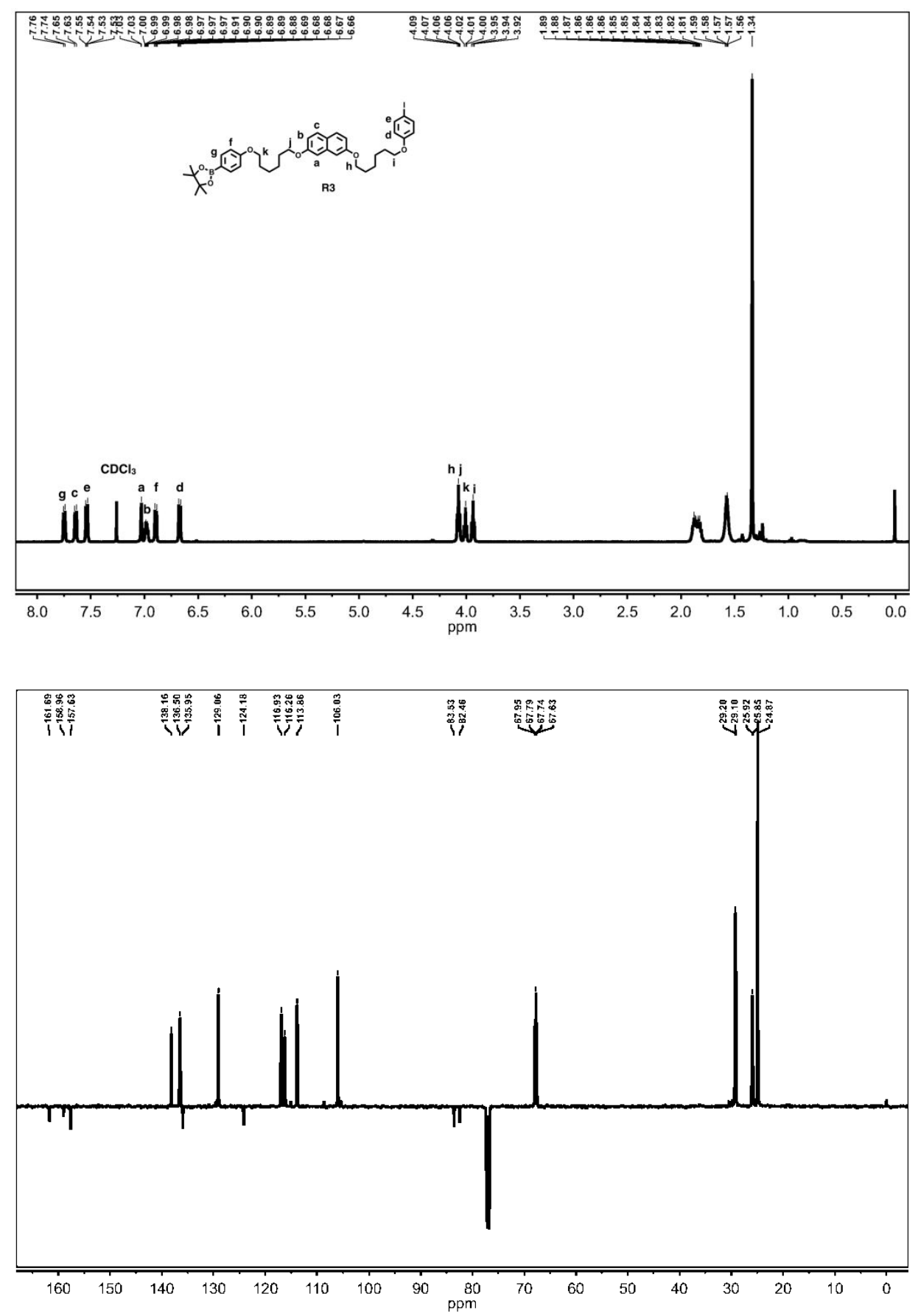

Figure S5. ${ }^{1} \mathrm{H}$ and ${ }^{13} \mathrm{C}$ NMR (DEPTQ) spectra of $\mathbf{R 3}$ in $\mathrm{CDCl}_{3}$. 

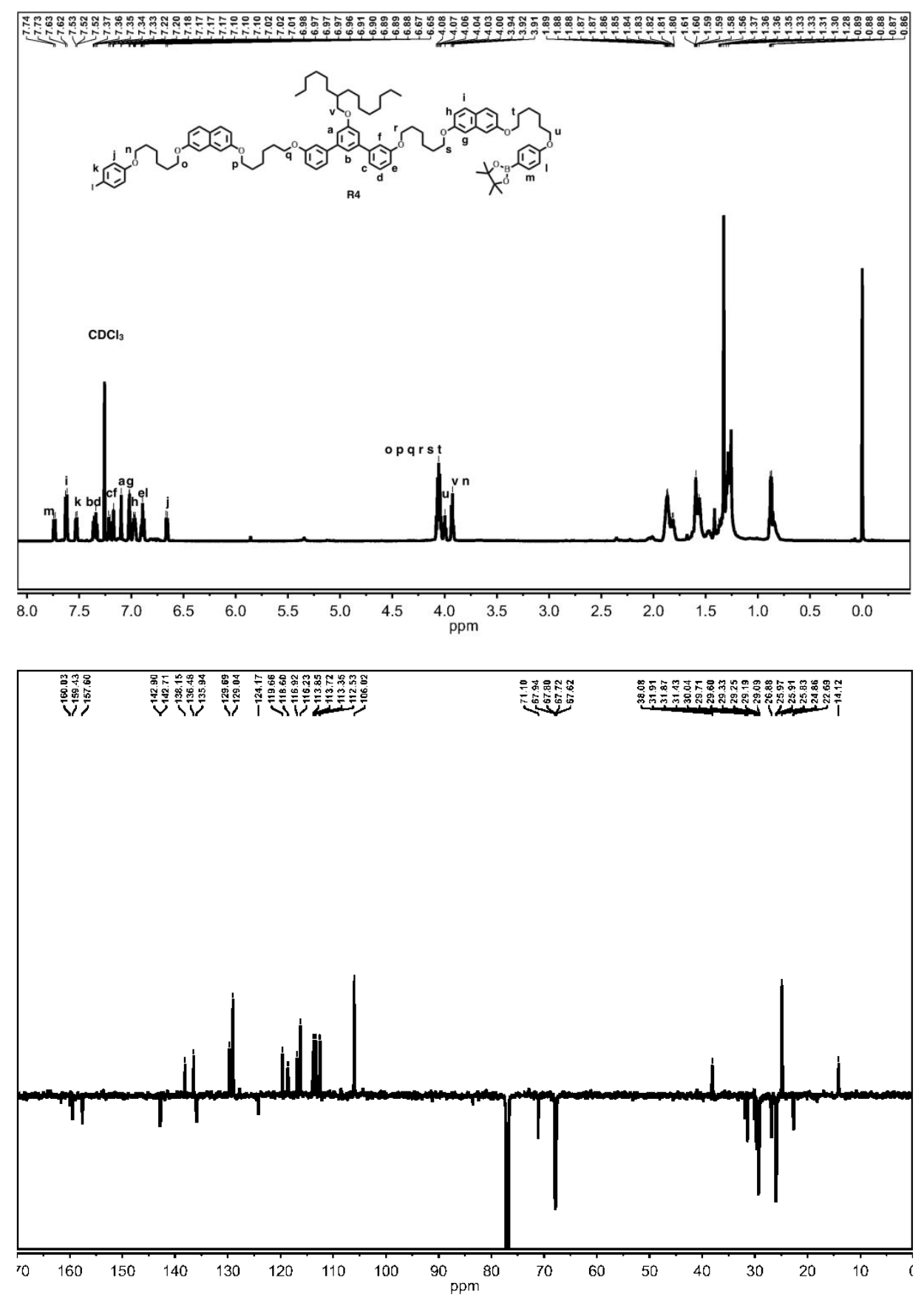

Figure S6. ${ }^{1} \mathrm{H}$ and ${ }^{13} \mathrm{C}$ NMR (DEPTQ) spectra of $\mathbf{R 4}$ in $\mathrm{CDCl}_{3}$. 

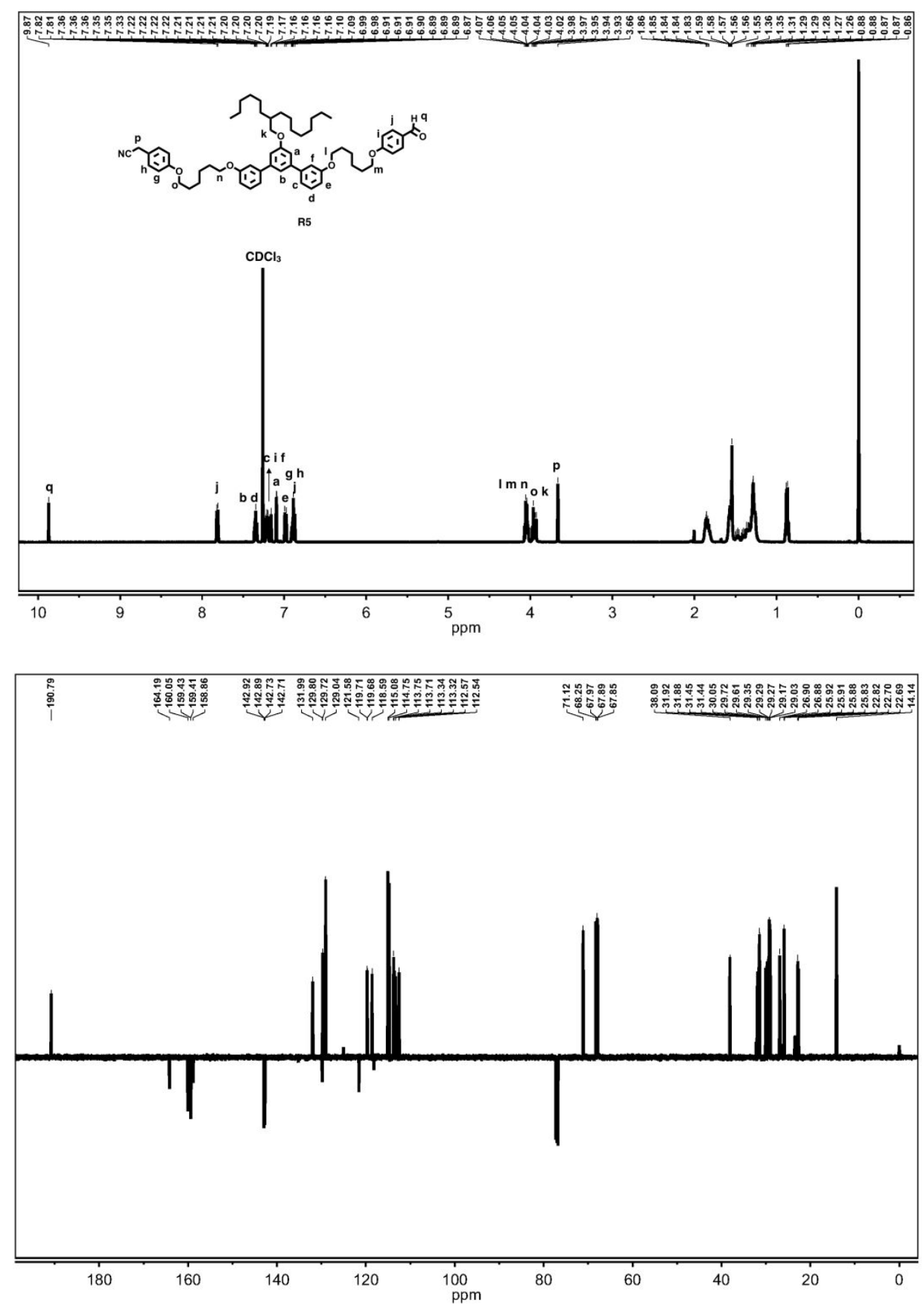

Figure S7. ${ }^{1} \mathrm{H}$ and ${ }^{13} \mathrm{C}$ NMR (DEPTQ) spectra of $\mathbf{R 5}$ in $\mathrm{CDCl}_{3}$. 

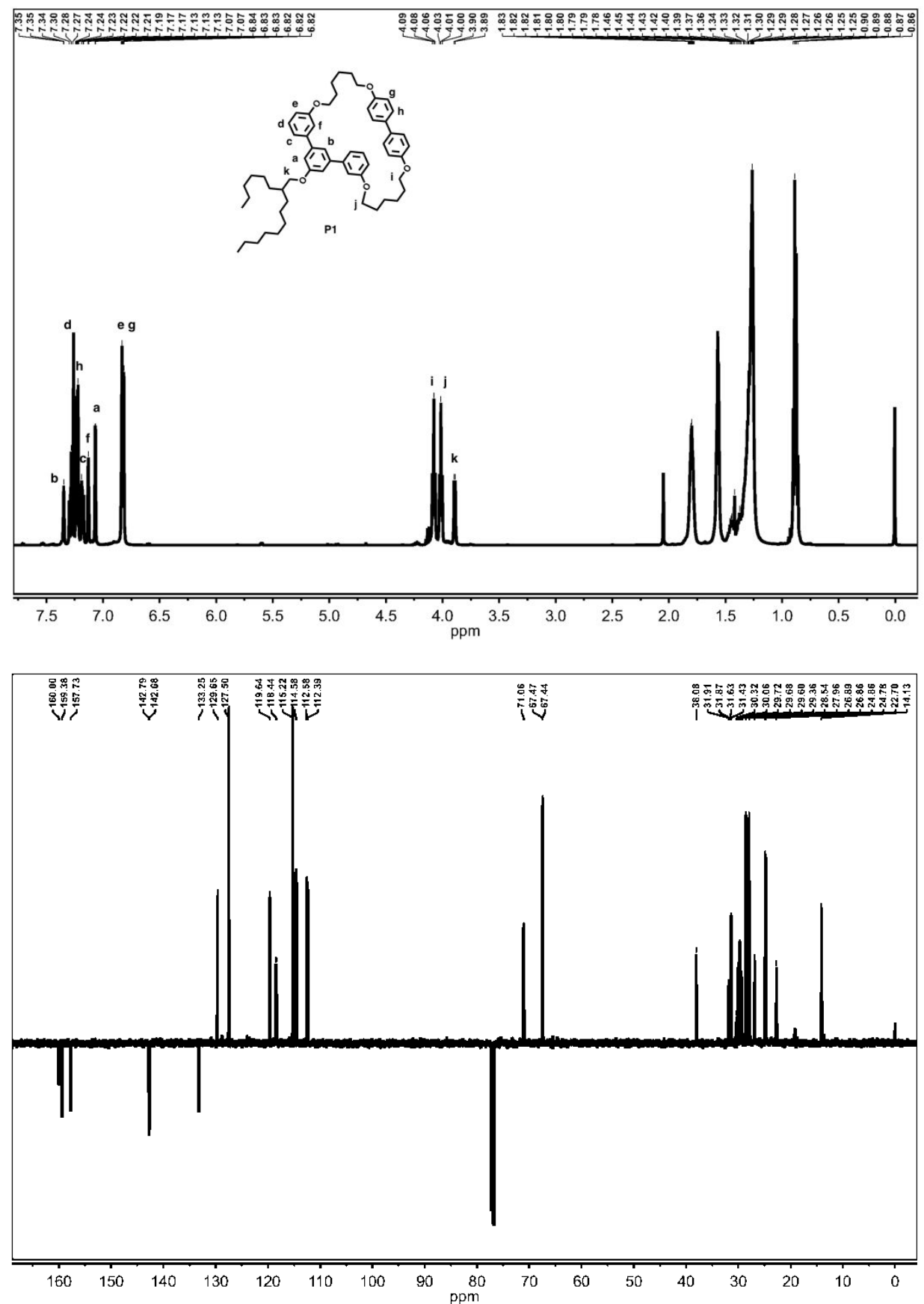

Figure S8. ${ }^{1} \mathrm{H}$ and ${ }^{13} \mathrm{C}$ (DEPTQ) spectra of $\mathbf{P 1}$ in $\mathrm{CDCl}_{3}$. 

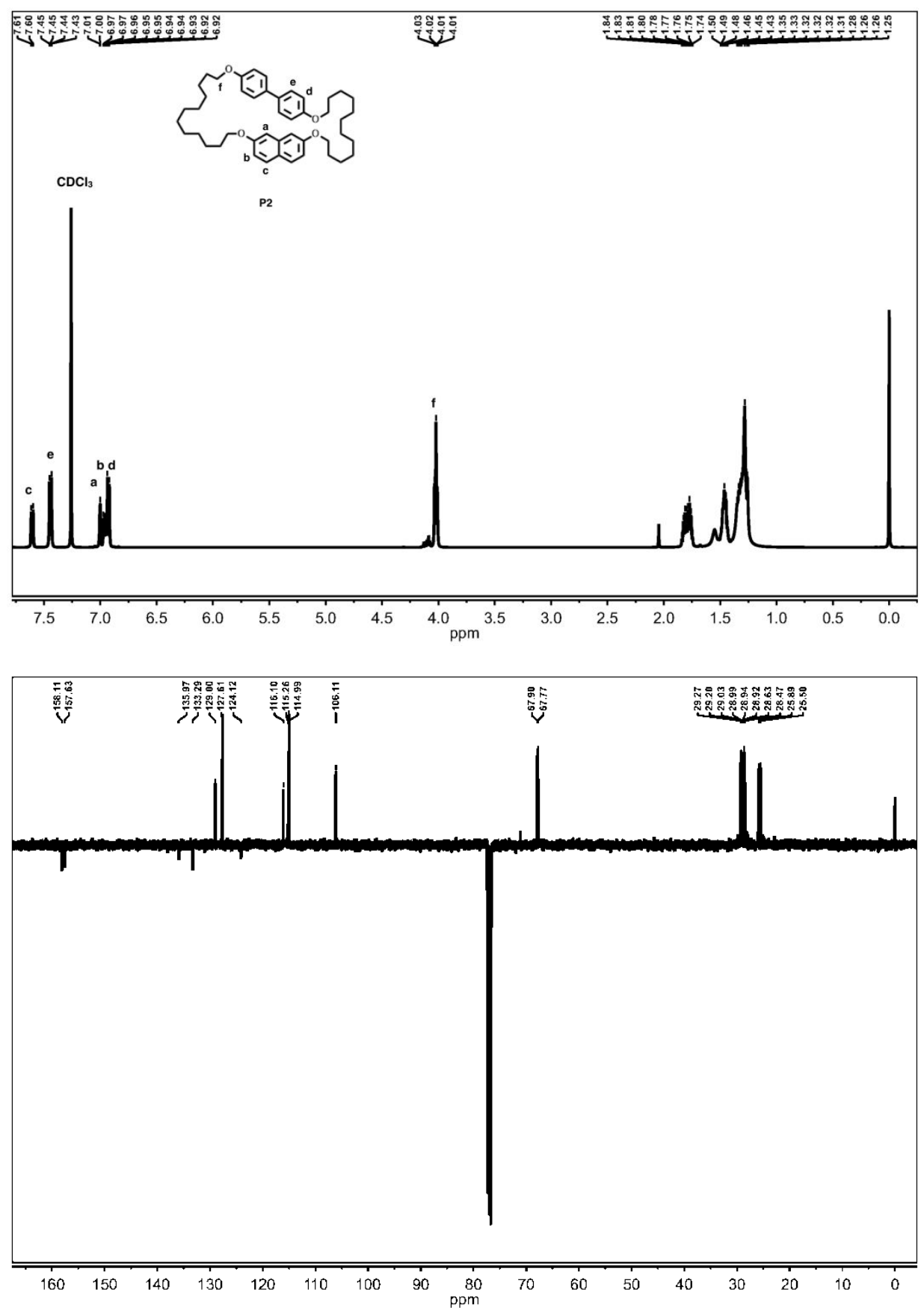

Figure S9. ${ }^{1} \mathrm{H}$ and ${ }^{13} \mathrm{C}$ (DEPTQ) spectra of $\mathbf{P} 2$ in $\mathrm{CDCl}_{3}$. 

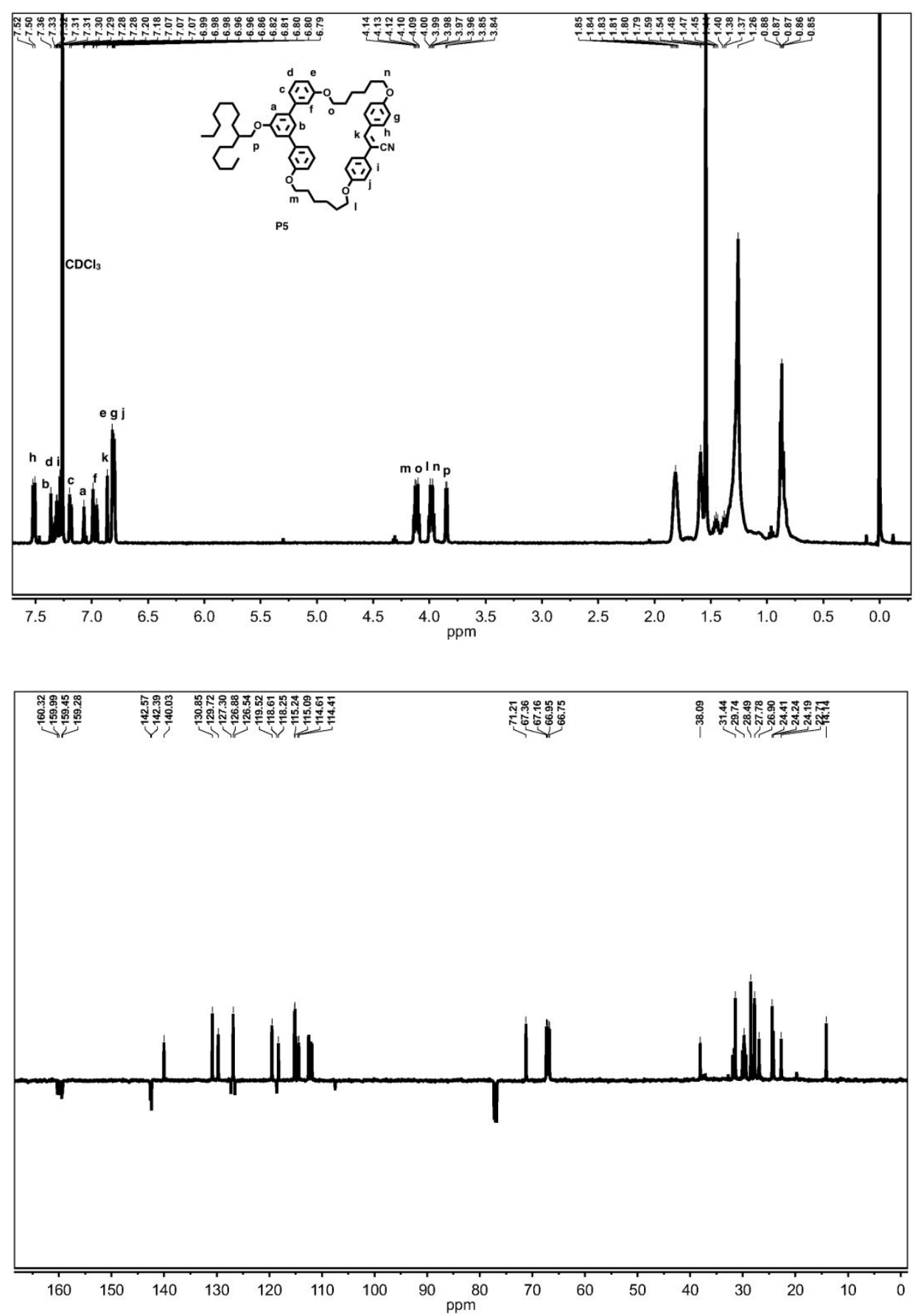

Figure S10. ${ }^{1} \mathrm{H}$ and ${ }^{13} \mathrm{C}$ (DEPTQ) spectra of $\mathbf{P 5}$ in $\mathrm{CDCl}_{3}$. 


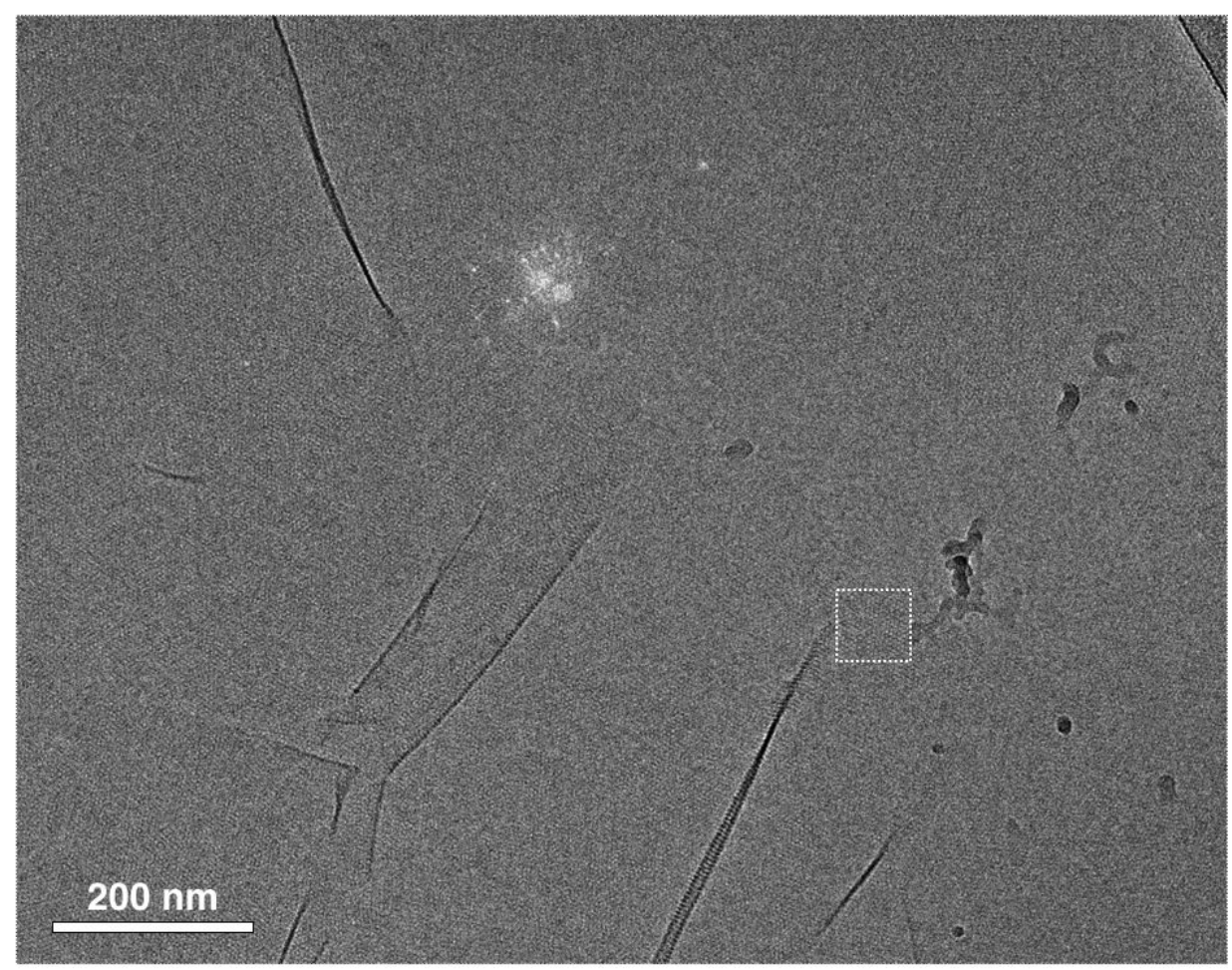

Figure S11. Large area cryo-TEM image of 1 in water-methanol solution (3/7, v/v). The magnified image corresponding to the area indicated by the white square is shown in Figure 2a, inset.

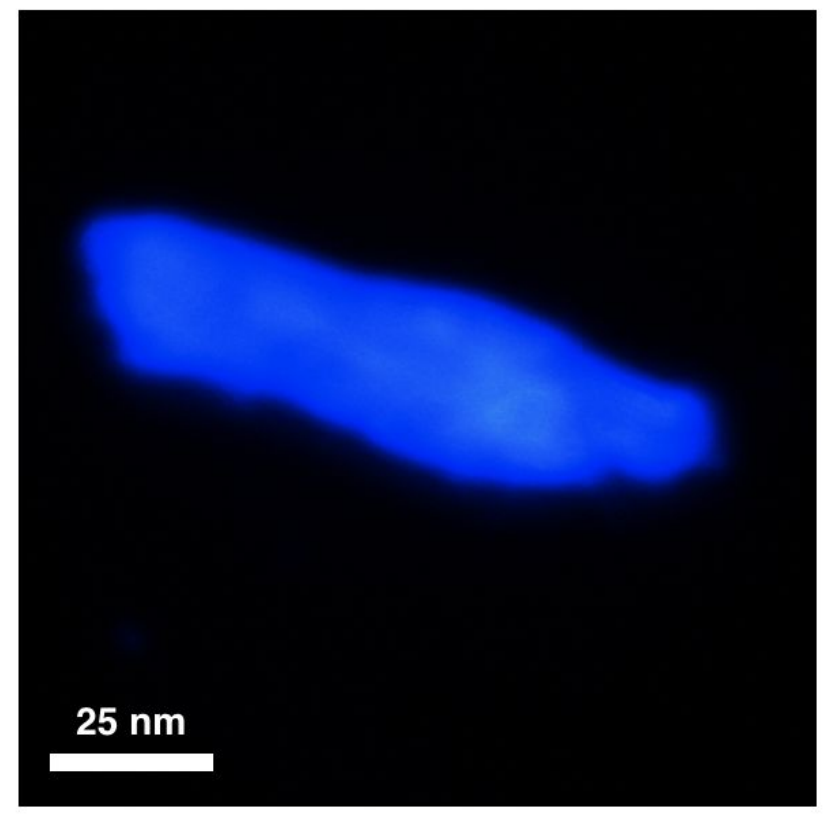

Figure S12. Fluorescence optical microscopy (FOM) image of 1 in water-methanol solution $(3 / 7, \mathrm{v} / \mathrm{v})$. 


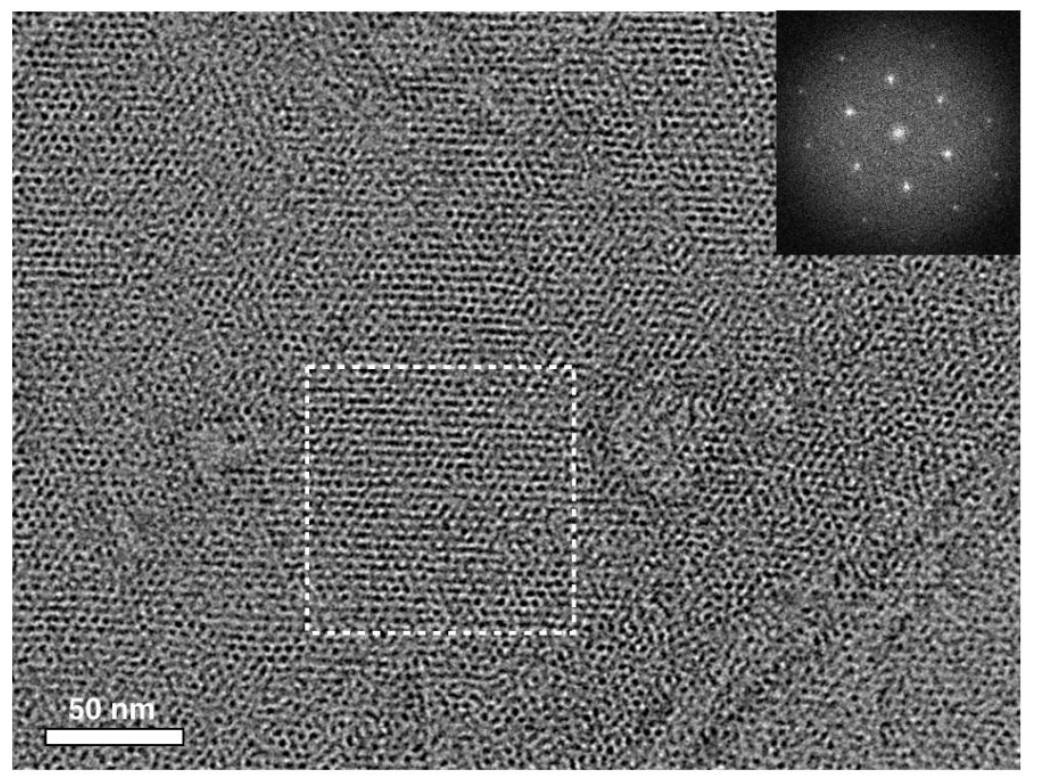

Figure S13. Large area TEM image of 1, cast from water-methanol solution (3/7, $\mathrm{v} / \mathrm{v}$ ). The magnified image corresponding to the area indicated by the white square is shown in Figure 2c. The inset shows 2D Fourier transformation of a hexagonal structure.
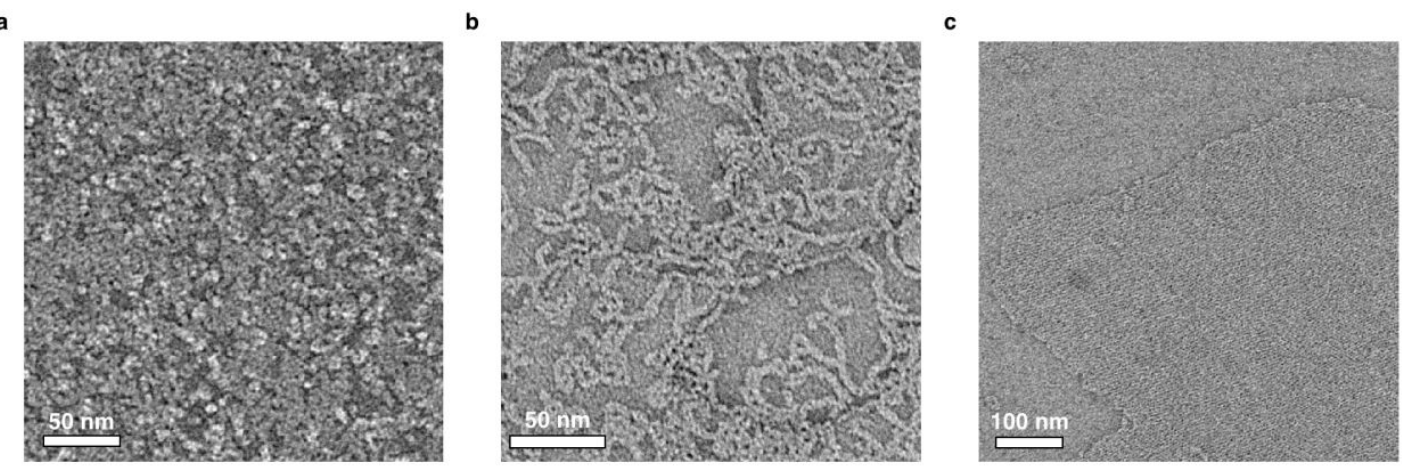

Figure S14. Negatively-stained TEM images with increasing concentration of $\mathbf{1}$, cast from water-methanol solution (3/7, v/v), $9.1 \mu \mathrm{M}$ (a), $18.2 \mu \mathrm{M}$ (b) and $45.5 \mu \mathrm{M}$ (c). 


\begin{tabular}{ccccc}
\hline $\mathrm{h}$ & $\mathrm{k}$ & $\mathrm{l}$ & $q_{\text {calad }}(\mathrm{nm}-1)$ & $q_{\text {obsd }}(\mathrm{nm}-1)$ \\
\hline 1 & 4 & 1 & 7.452 & 7.435 \\
2 & 0 & 0 & 7.832 & 7.811 \\
1 & 1 & 0 & 7.832 & 7.811 \\
1 & 1 & 5 & 8.076 & 8.055 \\
2 & 3 & 3 & 8.303 & 8.318 \\
0 & 0 & 6 & 9.110 & 9.103 \\
2 & 4 & 3 & 9.577 & 9.586 \\
1 & 2 & 6 & 10.043 & 10.049 \\
1 & 6 & 0 & 10.439 & 10.459 \\
3 & 0 & 0 & 11.678 & 11.673 \\
0 & 5 & 5 & 11.003 & 11.006 \\
1 & 6 & 4 & 12.075 & 12.082 \\
4 & 5 & 1 & 12.526 & 12.534 \\
1 & 7 & 3 & 12.849 & 12.84 \\
0 & 2 & 0 & 13.387 & 13.413 \\
5 & 1 & 0 & 13.672 & 13.680 \\
1 & 2 & 0 & 13.956 & 14.01 \\
0 & 6 & 7 & 14.296 & 14.300 \\
2 & 2 & 9 & 14.744 & 14.741 \\
5 & 6 & 0 & 15.192 & 15.196 \\
4 & 0 & 0 & 15.665 & 15.648 \\
4 & 2 & 9 & 16.059 & 16.063 \\
5 & 5 & 6 & 16.533 & 16.527 \\
4 & 1 & 0 & 16.947 & 16.939 \\
10 & 1 & 3 & 17.383 & 17.394 \\
3 & 2 & 0 & 17.8 & 17.788 \\
10 & 3 & 2 & 19.021 & 19.027 \\
5 & 0 & 0 & 19.509 & 19.523 \\
\hline & & & & \\
& & & &
\end{tabular}

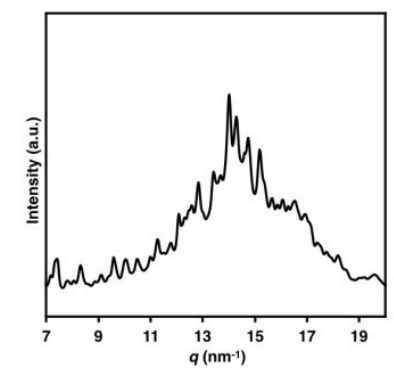

Top View:

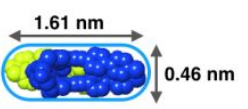

Side View:

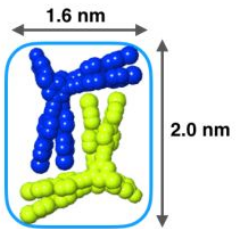

Figure S15. Wide-angle X-ray diffraction (WAXS) data of 1 fitting into a 2D face-centered rectangular structure. $q_{\text {calcd }}$ and $q_{\text {obsd }}$ are the scattering vectors of the calculated and the observed reflections for the 2D face-centered rectangular structure with lattice parameters, $a=1.61 \mathrm{~nm}$ and $b=0.92 \mathrm{~nm}$. Right column: WAXS reflections of $\mathbf{1}$ (above), the calculated dimensions of a faced dimer of $\mathbf{1}$ (below). 

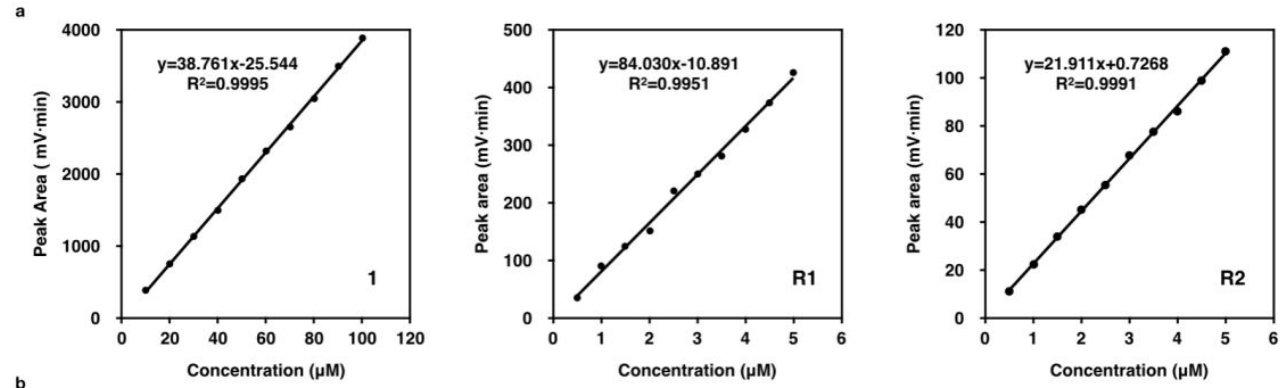

\begin{tabular}{|c|c|c|c|c|}
\hline \multicolumn{2}{|r|}{ 1-R1 } & 1st & 2nd & 3 rd \\
\hline \multirow{2}{*}{1} & $\begin{array}{l}\text { Peak area } \\
\text { (mV·min) }\end{array}$ & 3200.027 & 1086.387 & 2349.063 \\
\hline & $\begin{array}{c}\text { Concentration } \\
(\mu \mathrm{M})\end{array}$ & 83.217 & 28.687 & 61.262 \\
\hline \multirow{2}{*}{ R1 } & $\begin{array}{l}\text { Peak area } \\
(\mathrm{mV} \cdot \mathrm{min})\end{array}$ & 357.664 & 117.087 & 266.281 \\
\hline & $\begin{array}{c}\text { Concentration } \\
(\mu \mathrm{M})\end{array}$ & 4.386 & 1.523 & 3.327 \\
\hline \multirow{3}{*}{ Uptake } & $\mathrm{R} 1 / 1$ & 0.0527 & 0.0531 & 0.0543 \\
\hline & R1/Pores & $94.86 \%$ & $95.58 \%$ & $97.74 \%$ \\
\hline & Average & \multicolumn{3}{|c|}{$96.06 \pm 1.12 \%$} \\
\hline
\end{tabular}

\begin{tabular}{|c|c|c|c|c|}
\hline \multicolumn{2}{|r|}{ 1-R2 } & 1st & 2nd & 3rd \\
\hline \multirow{2}{*}{1} & $\begin{array}{c}\text { Peak area } \\
(\mathrm{mV} \cdot \mathrm{min})\end{array}$ & 1303.868 & 2514.993 & 2644.009 \\
\hline & $\begin{array}{c}\text { Concentration } \\
(\mu \mathrm{M})\end{array}$ & 34.298 & 65.542 & 68.872 \\
\hline \multirow{2}{*}{ R2 } & $\begin{array}{l}\text { Peak area } \\
(\mathrm{mV} \cdot \mathrm{min})\end{array}$ & 40.868 & 76.122 & 90.606 \\
\hline & $\begin{array}{c}\text { Concentration } \\
(\mu \mathrm{M})\end{array}$ & 1.862 & 3.441 & 4.102 \\
\hline \multirow{3}{*}{ Uptake } & $\mathrm{R} 2 / 1$ & 0.0543 & 0.0525 & 0.0527 \\
\hline & R2/Pores & $96.16 \%$ & $94.52 \%$ & $94.97 \%$ \\
\hline & Average & \multicolumn{3}{|c|}{$95.21 \pm 0.48 \%$} \\
\hline
\end{tabular}
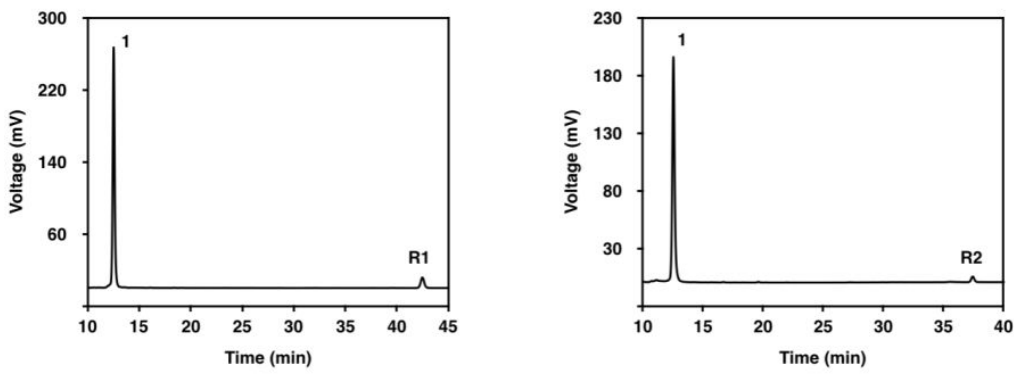

Figure S16. (a) Calibration curves for quantifications using HPLC analysis of 1, R1 and R2. (b) Analytic tables for the uptake of 1-R1 and 1-R2. (c) HPLC spectra for the uptake of $\mathbf{R} 1$ and $\mathbf{R} 2$. 


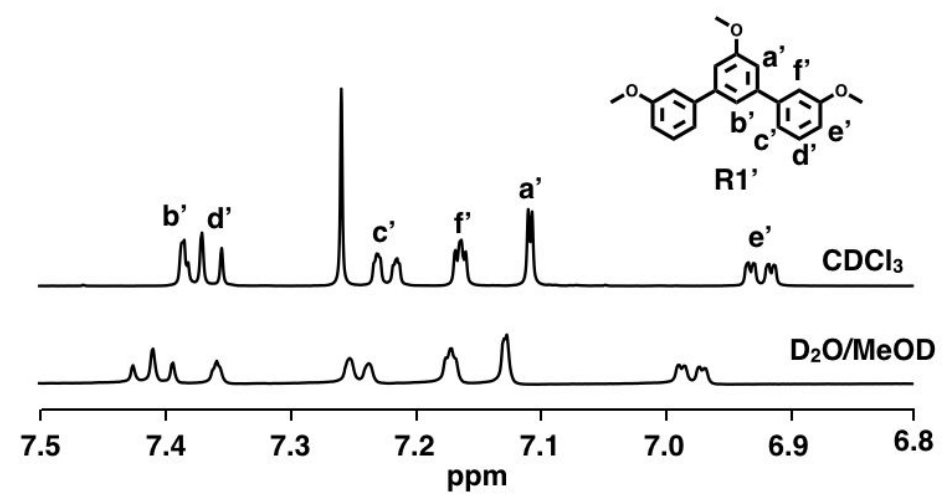

Figure S17. ${ }^{1} \mathrm{H}-\mathrm{NMR}$ of $\mathbf{R 1}$ ' in $\mathrm{CDCl}_{3}$ and in $\mathrm{D}_{2} \mathrm{O} / \mathrm{MeOD}(3 / 7$, v/v) with same concentration.
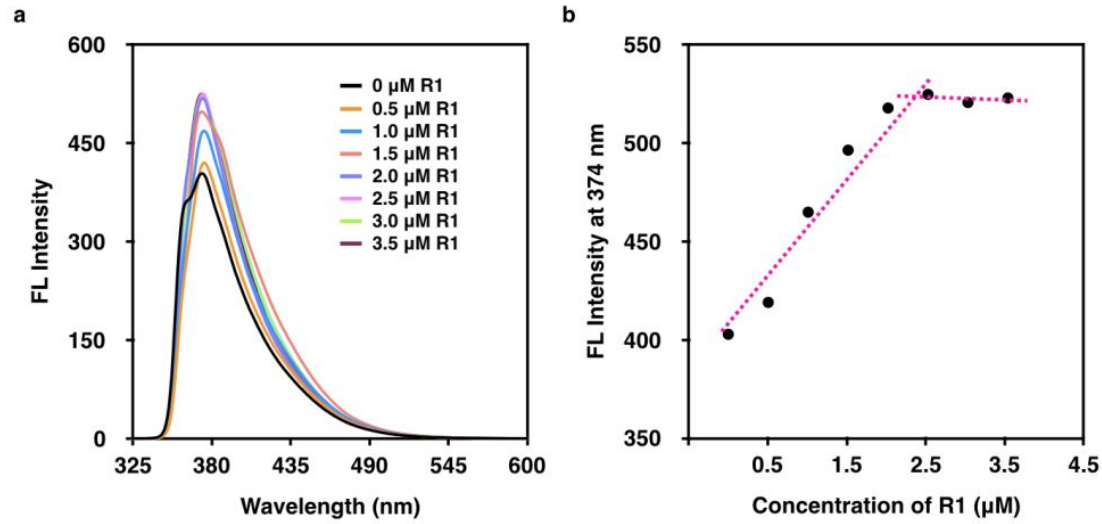

Figure S18. (a) Fluorescence spectra from different concentrations of R1 based on 45 $\mu \mathrm{M}$ of 1 in water-methanol solution $(3 / 7, \mathrm{v} / \mathrm{v})$. Excitation wavelength: $320 \mathrm{~nm}$. (b) Fluorescence intensity dependence at $374 \mathrm{~nm}$ on the different concentrations of R1. Cross point indicates the saturation point is $2.5 \mu \mathrm{M}$ of $\mathbf{R} \mathbf{1}$. Considering one pore consisting of 18 molecules of $\mathbf{1}$, the result demonstrates that each pore includes one substrate R1. To rule out the influence of the guest on the fluorescence intensity of $\mathbf{1}$, the excitation wavelength at $320 \mathrm{~nm}$ was selected, which is not influenced by the substrate. 


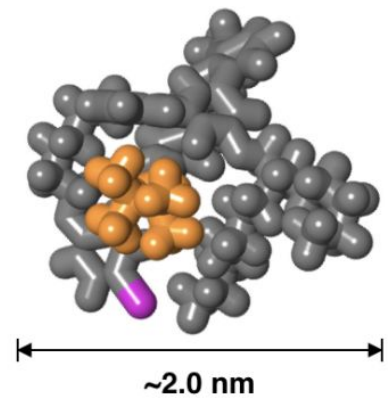

R1

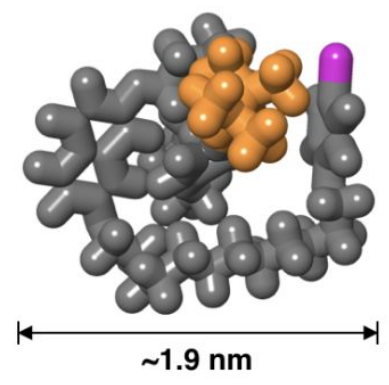

R2

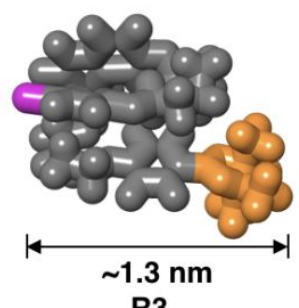

R3

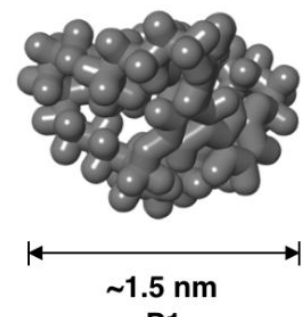

P1

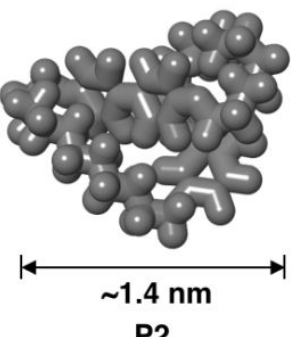

P2

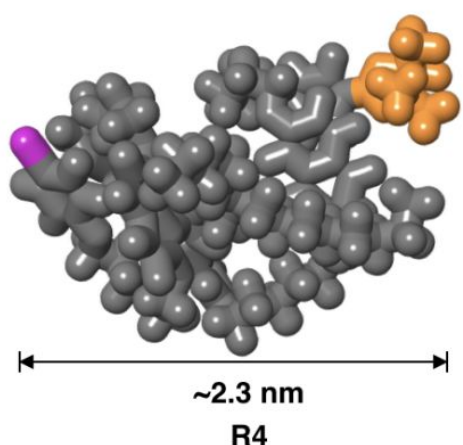

R4

Figure S19. Molecular dynamic simulations for the size of reactants and products (R1, R2, R3, R4, P1 and P2) in a folded conformation formed in aqueous condition. 

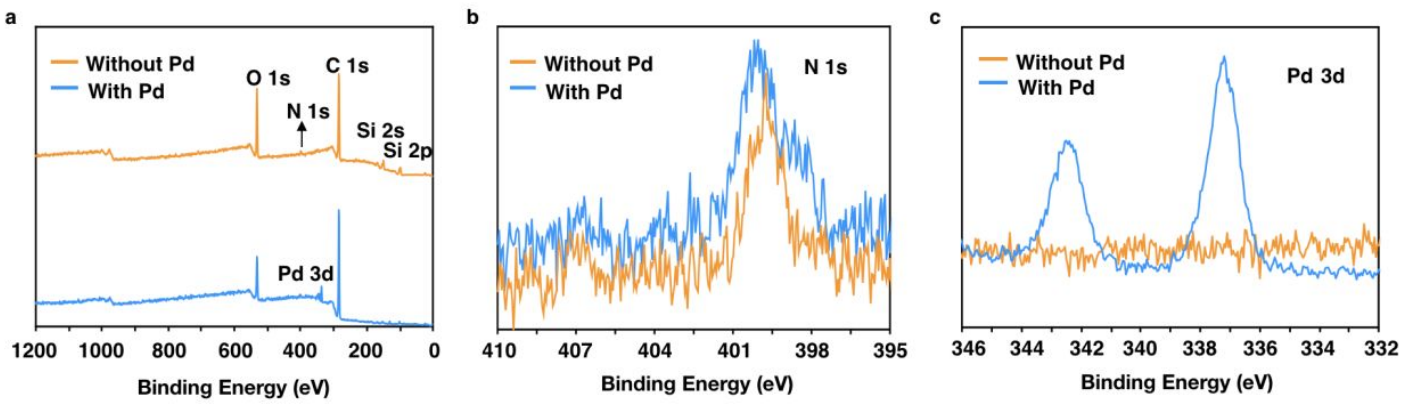

Figure S20. The XPS spectra of 1 and $\operatorname{Pd}(0)$ coordinated 1. (a) Survey. (b) $\mathrm{N}_{1 \mathrm{~s}}$. The nitrogen (1s) peak showed a positive shift from $399.3 \mathrm{eV}$ to $400.1 \mathrm{eV}$ after the addition of $\operatorname{Pd}(0)$. (c) $\operatorname{Pd}_{3 \mathrm{~d}}$.

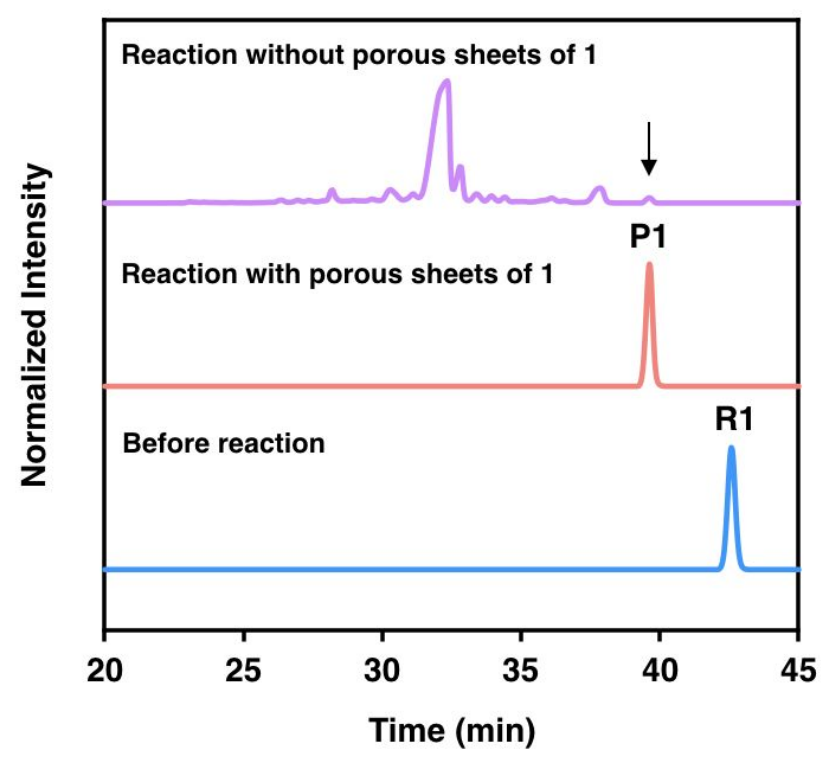

Figure S21. HPLC chromatograms for the conversion of R1 for the Suzuki-Miyaura coupling reactions in toluene/EtOH solution in the absence of the porous nanosheets of $\mathbf{1}$ after $48 \mathrm{hr}$ at $75{ }^{\circ} \mathrm{C}$ (violet), the conversion of $\mathbf{R} \mathbf{1}$ in the presence of the porous nanosheets of 1 in water-methanol (3/7, v/v) solution after $4 \mathrm{hr}$ at room temperature (red), and $\mathbf{R} 1$ before the reaction in water-methanol (3/7, v/v) solution (blue). The arrow in violet indicates that the cyclized product is formed below $1 \%$ yield. 

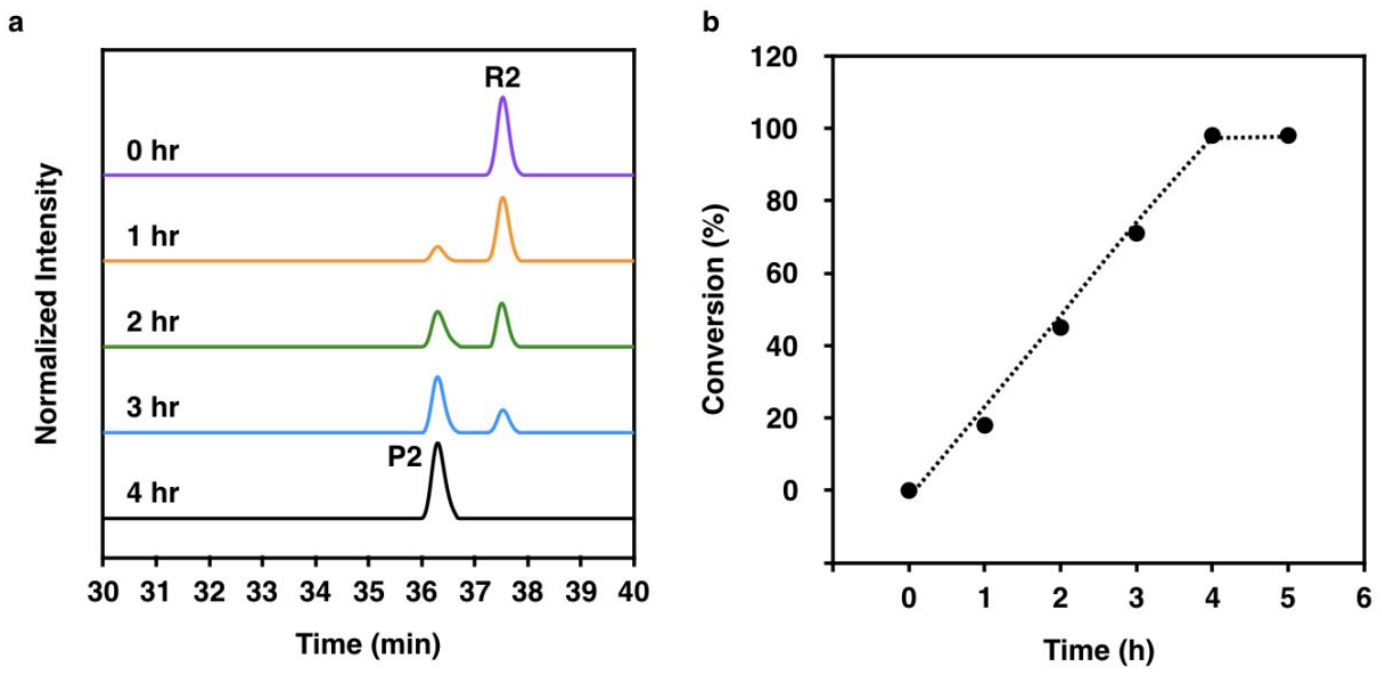

Figure S22. (a) HPLC chromatograms obtained from macrocyclization of $\mathbf{R} 2$ in the porous nanosheets of $\mathbf{1}$ with time variation. (b) The conversion of $\mathbf{R} \mathbf{2}$ in the presence of the porous nanosheets of $\mathbf{1}(91 \mu \mathrm{M})$ as a function of time in water-methanol solution $(3 / 7, \mathrm{v} / \mathrm{v})$.
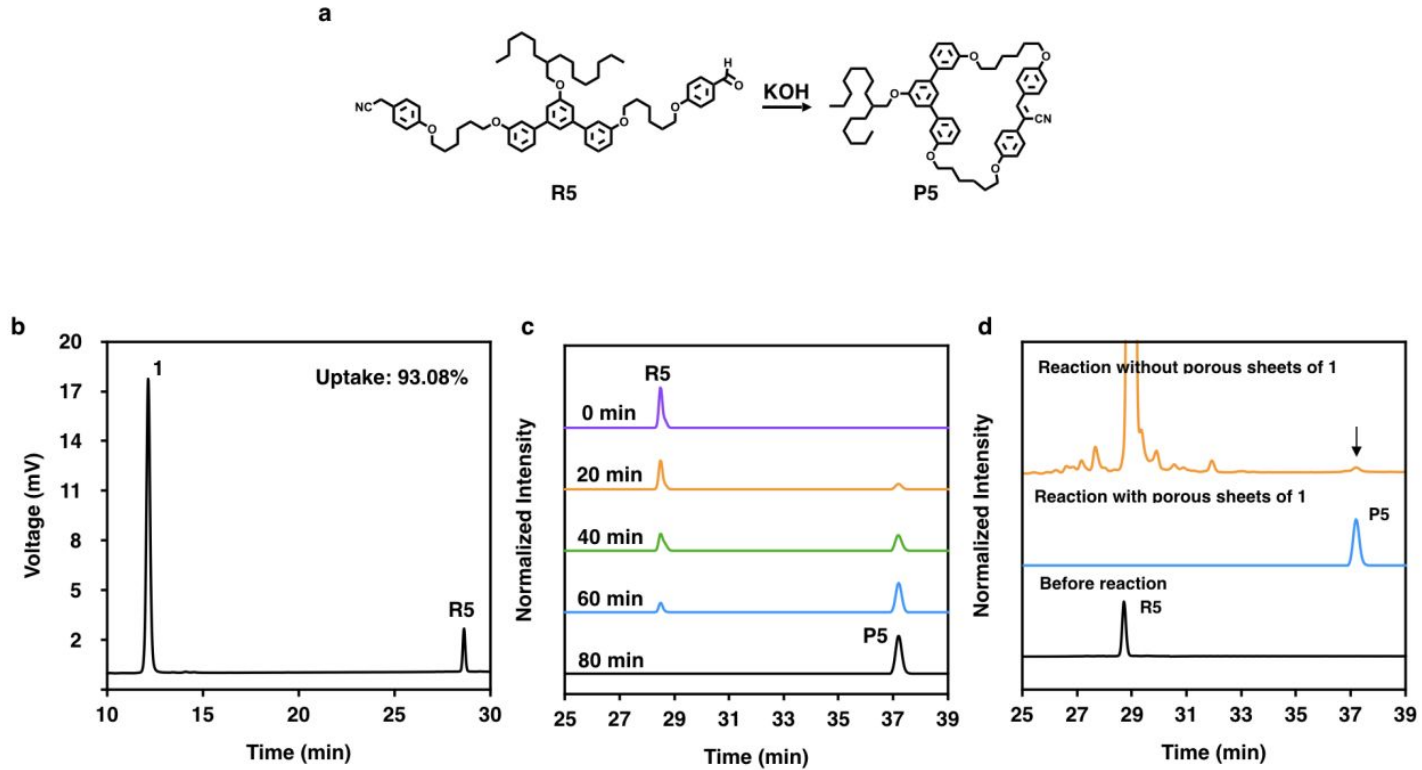

Figure S23. (a) Chemical structure of R5 and P5. (b) HPLC spectra for the uptake of R5. (c) HPLC chromatograms obtained from macrocyclization of $\mathbf{R 5}$ in the porous nanosheets of 1 with time variation. (d) HPLC chromatograms for the conversion of R5 for the Knoevenagel condensation reactions in $\mathrm{MeOH} / \mathrm{CHCl}_{2}$ solution in the absence of the porous nanosheets of 1 after $80 \mathrm{~min}$ at $60{ }^{\circ} \mathrm{C}$ (orange), the conversion of $\mathbf{R 5}$ in the presence of the porous nanosheets of $\mathbf{1}$ in water-methanol (3/7, v/v) solution after $80 \mathrm{~min}$ at room temperature (blue), and $\mathbf{R 5}$ before the reaction in water-methanol $(3 / 7, \mathrm{v} / \mathrm{v})$ solution (black). 


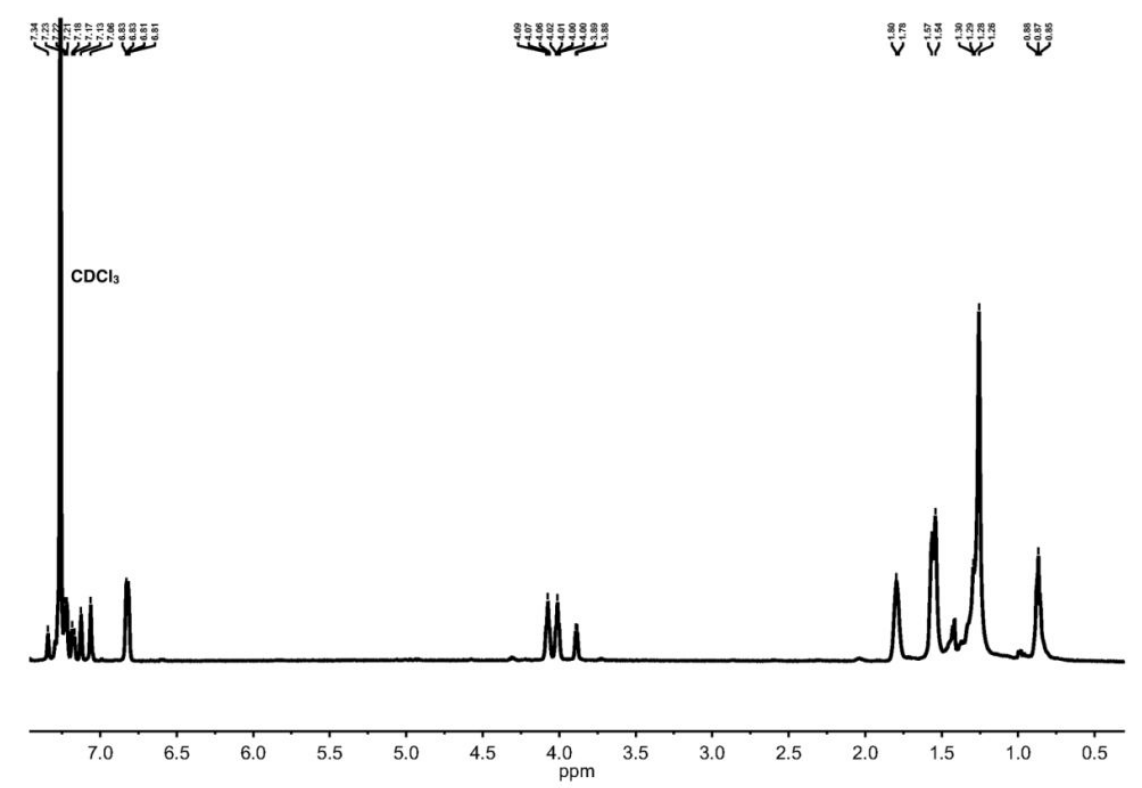

Figure S24. ${ }^{1} \mathrm{H}$ NMR spectrum obtained from the precipitate after complete conversion of $\mathbf{R} \mathbf{1}$ in the porous nanosheets of $\mathbf{1}$ in water-methanol solution (3/7, v/v). The result shows that the precipitate consists of a pure macrocycle product without any noticeable linear side products.

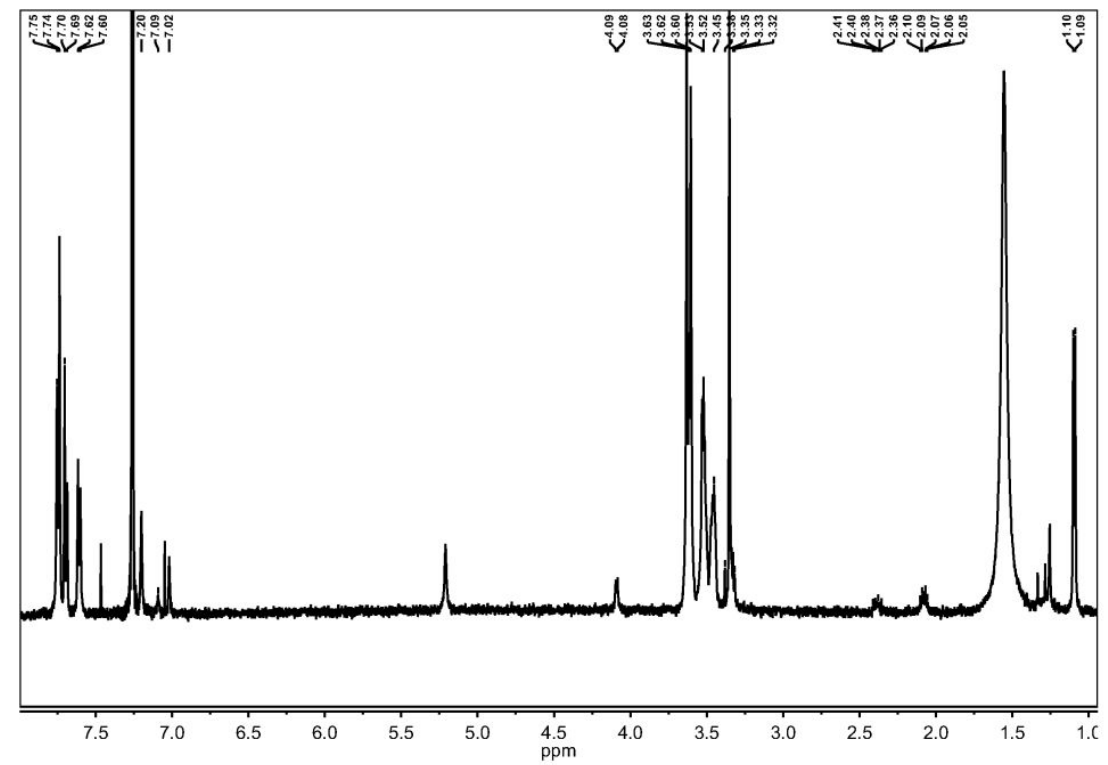

Figure S25. ${ }^{1} \mathrm{H}$ NMR spectrum obtained from the supernatant after complete conversion of $\mathbf{R} \mathbf{1}$ in the porous nanosheets of $\mathbf{1}$ in water-methanol solution (3/7, v/v) and removing solvent (in $\mathrm{CDCl}_{3}$ ). The result shows that the supernatant consists of pure molecule 1 without any noticeable cyclic products. 


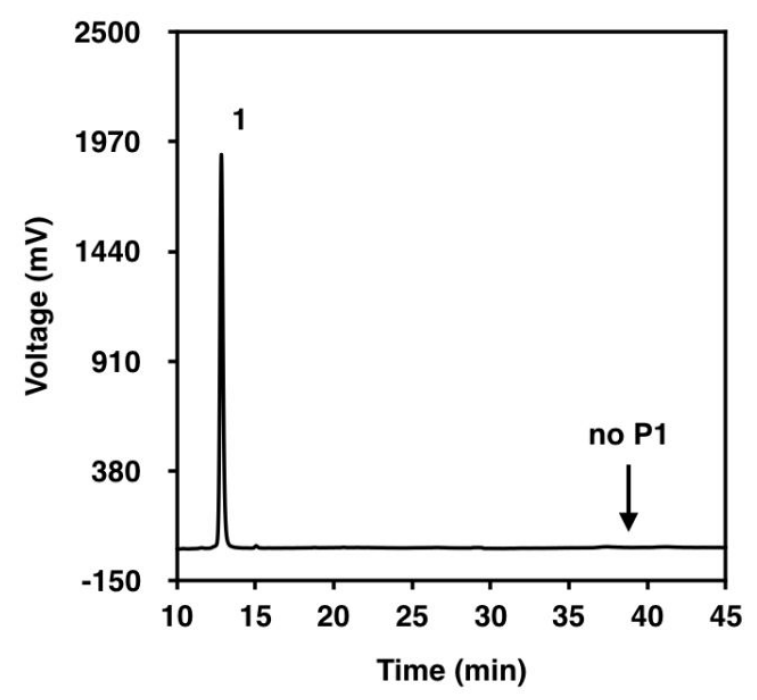

Figure S26. HPLC chromatogram of the porous nanosheet solution after removal of the precipitated macrocycle product $\mathbf{P 1}$ by decanting. The chromatogram shows a lack of any noticeable cyclic product $\mathbf{P 1}$.

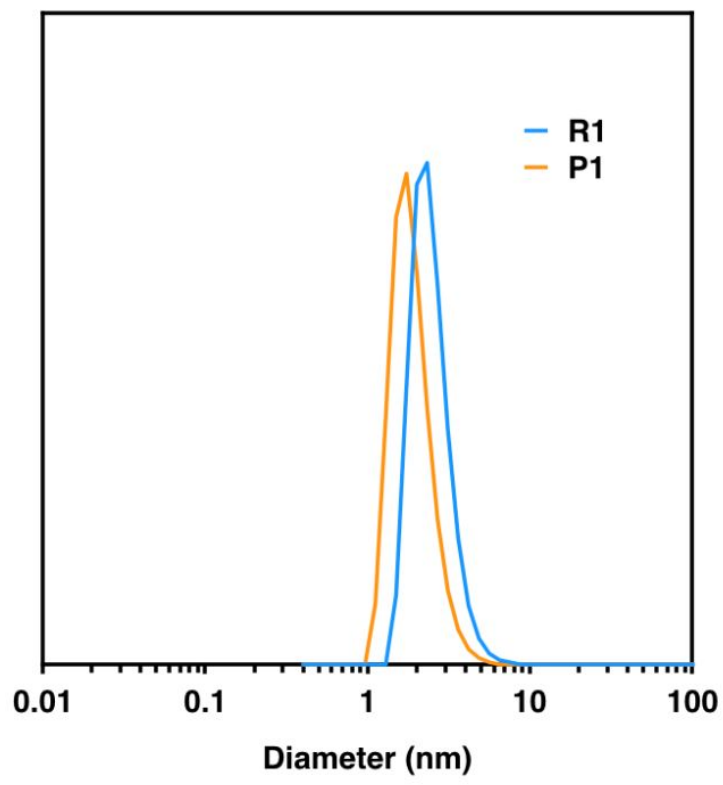

Figure S27. DLS profiles of R1 $(50 \mu \mathrm{M})$ and $\mathbf{P 1}(50 \mu \mathrm{M})$ in chloroform solution. Hydrodynamic diameter of $\mathbf{R} \mathbf{1}=2.6 \mathrm{~nm}$, and $\mathbf{P 1}=1.9 \mathrm{~nm}$. 


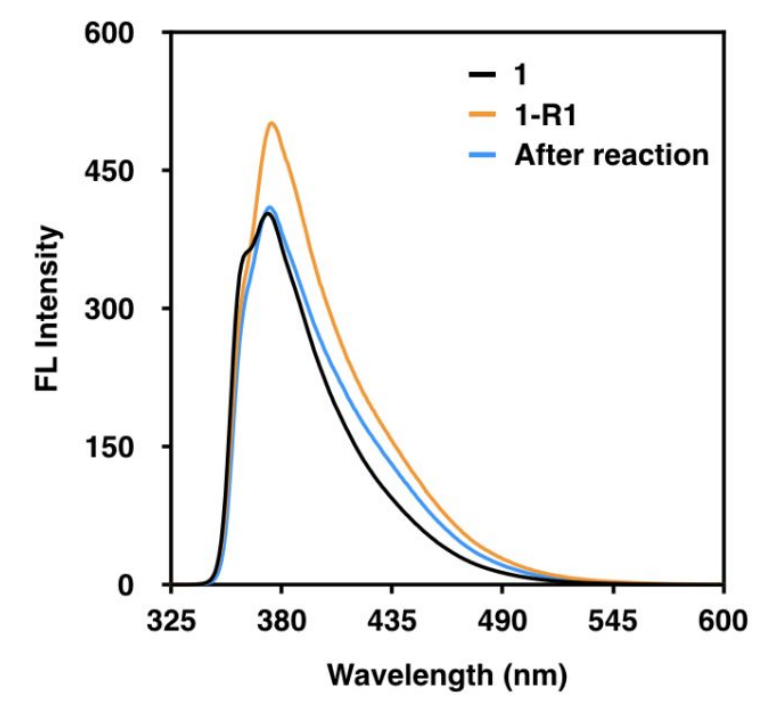

Figure S28. Fluorescence spectra obtained from 1, 1-R1 and the solution after reaction in water-methanol solution $(3 / 7, \mathrm{v} / \mathrm{v})$. Excitation wavelength: $320 \mathrm{~nm}$. The result indicates that the lack of interaction between the macrocycle product and pore after reaction.

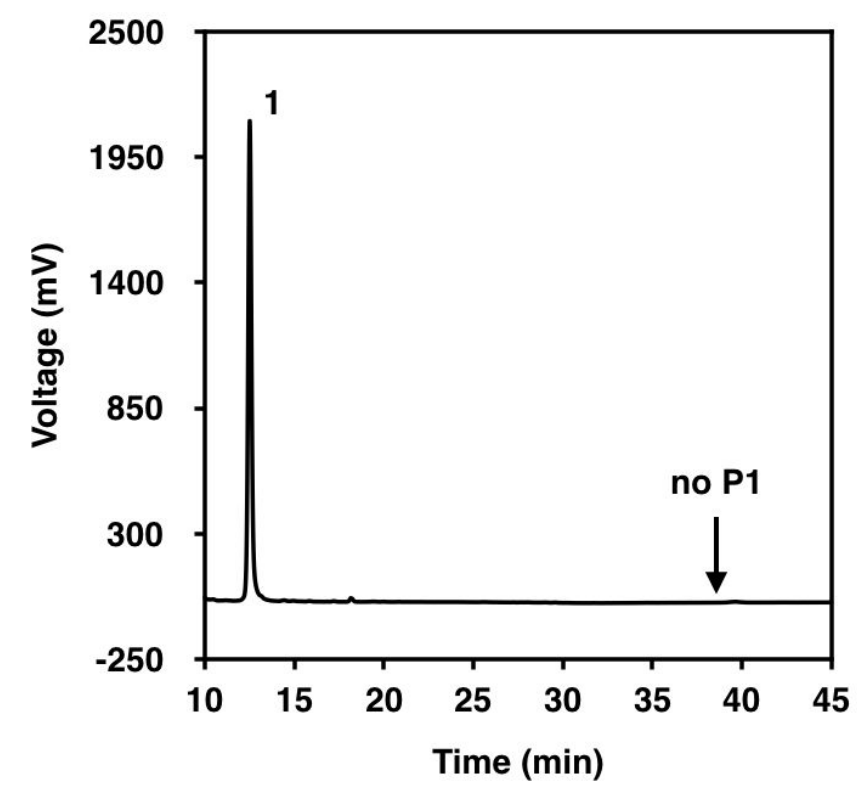

Figure S29. HPLC spectrum for the uptake of $\mathbf{P 1}$ in the porous nanosheets of $\mathbf{1}$ in water-methanol solution $(3 / 7, \mathrm{v} / \mathrm{v})$. The spectrum shows no any noticeable trace of $\mathbf{P 1}$, indicating that the porous nanosheets of $\mathbf{1}$ are unable to entrap the cyclic product due to the size mismatch between $\mathbf{P 1}$ and internal cavity of the porous nanosheets. 

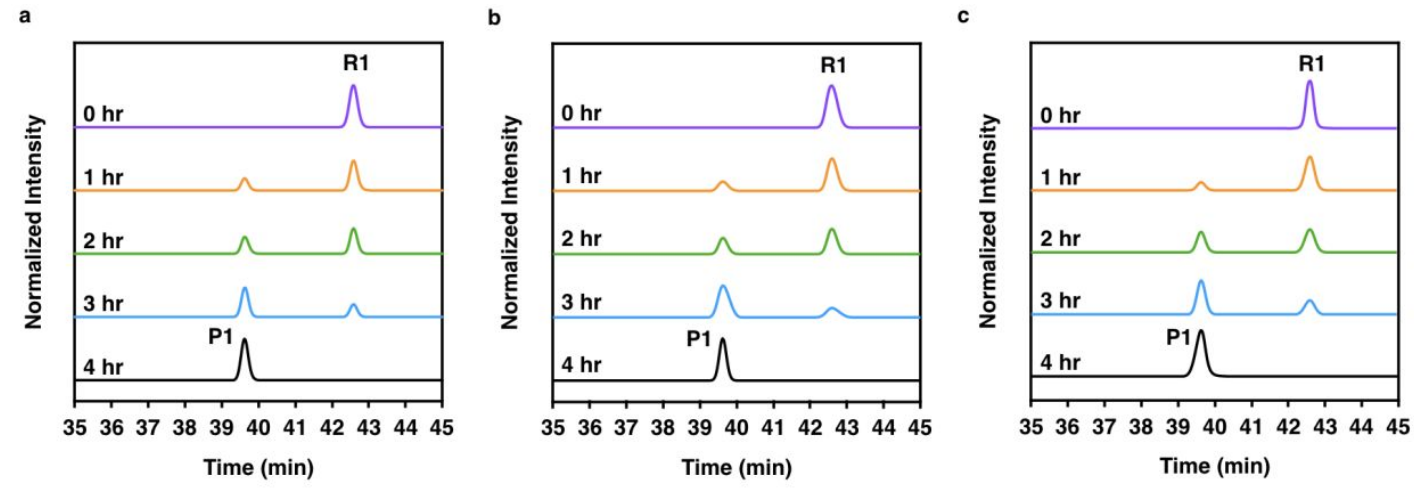

Figure S30. HPLC chromatograms of subsequent macrocyclizations of R1; the 2nd cycle (a), the 3 rd cycle (b) and the 4th cycle (c) in the presence of the porous nanosheets of 1 with time variation.

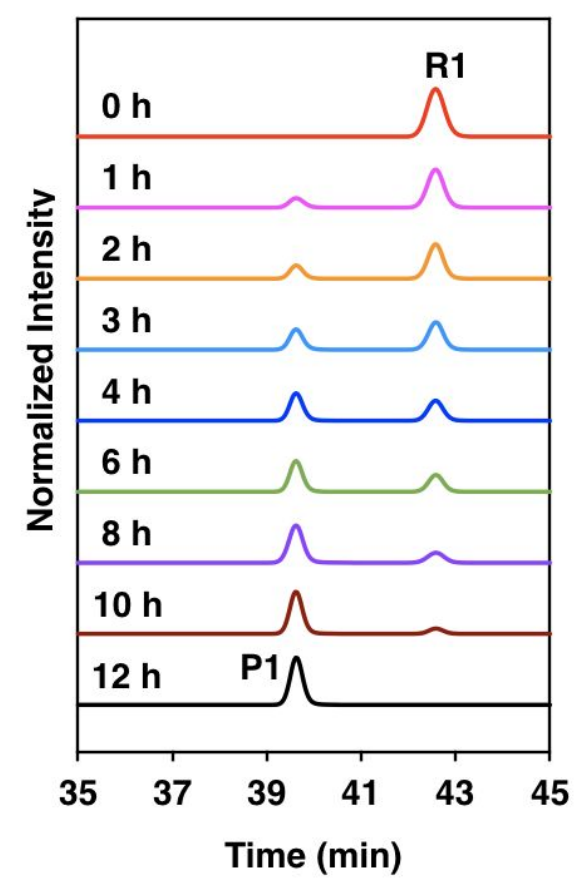

Figure S31. HPLC chromatograms of macrocyclization of excess amount of R1 (10 $\mu \mathrm{M})$ in the porous nanosheets of $\mathbf{1}(91 \mu \mathrm{M})$ without removal of untrapped $\mathbf{R} \mathbf{1}$ with time variation. The result showed that the cyclization undergoes consecutively until the excess substrate converts completely into the cyclic product even without the separation-out of the product. 

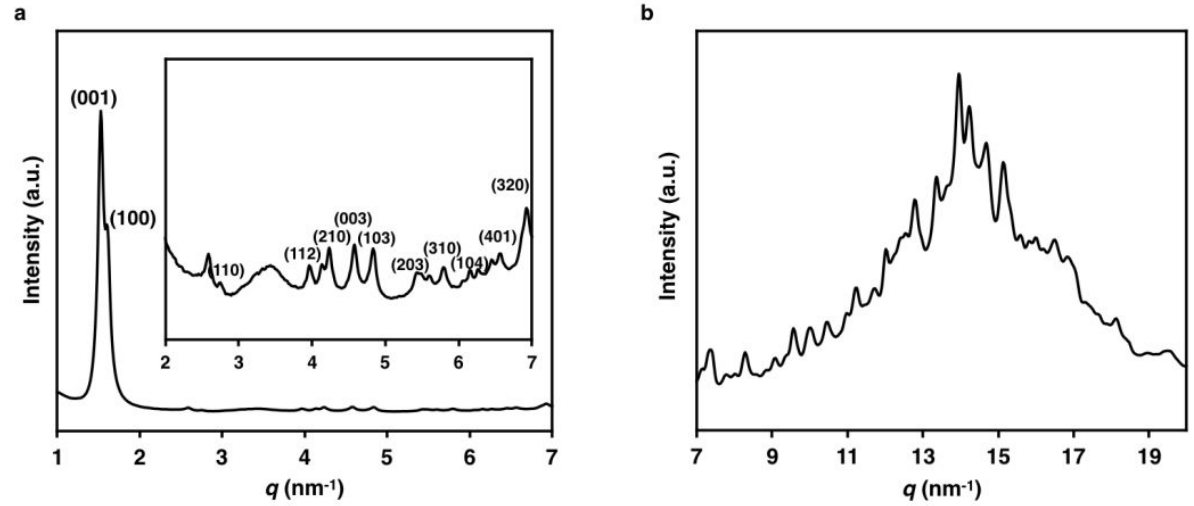

Figure S32. SAXS (a) and WAXS (b) patterns of the freeze-dried sample of 1 after performing 4 cycles of macrocyclizations of $\mathbf{R} \mathbf{1}$ in water-methanol (3/7, v/v). The results show that the porous nanosheet structures maintain without any structural deformation even after repeated cycles of macrocyclization.

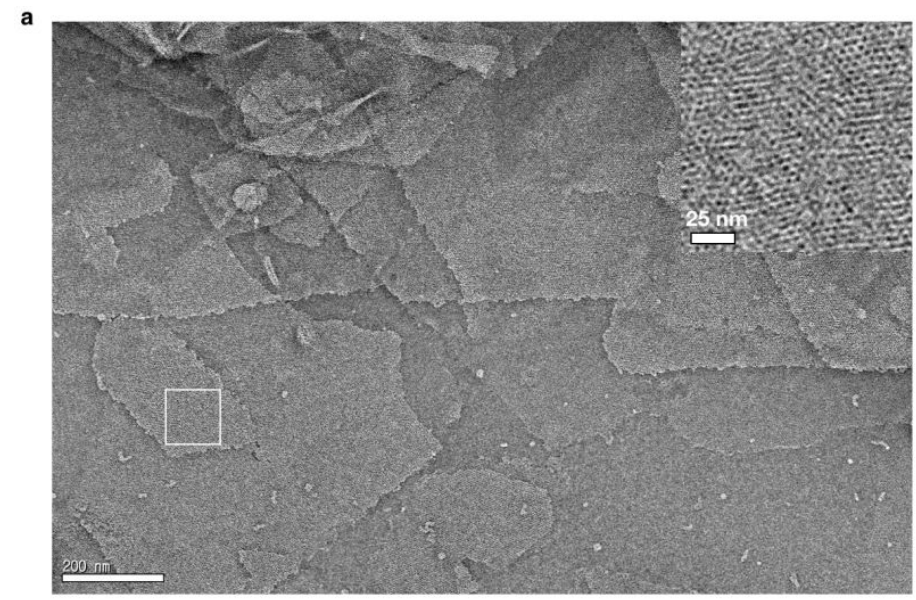

b

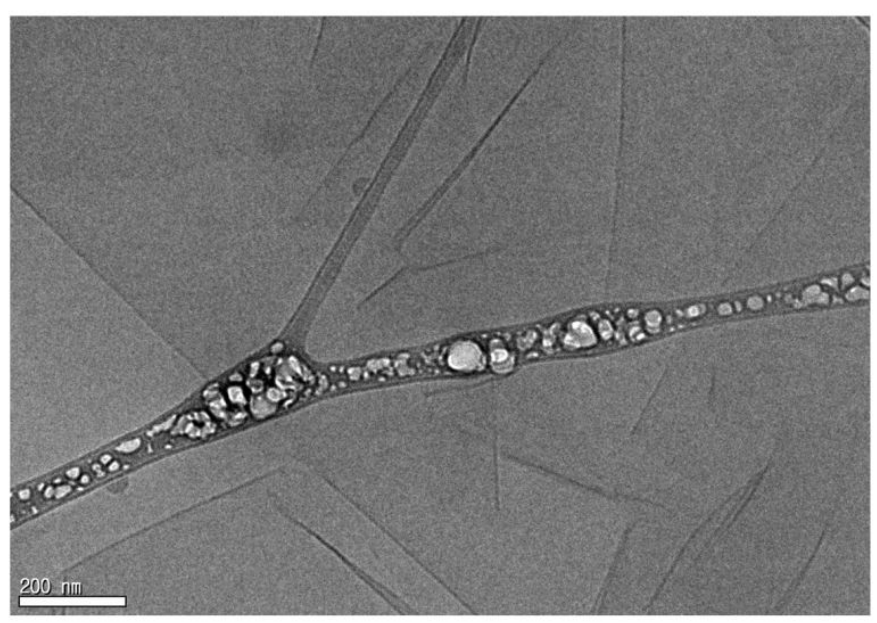

Figure S33. Negatively-stained TEM image (a) and cryo-TEM image (b) of 1 after 4 cycles of macrocyclization of R1. The images show that the porous nanosheet structures maintain without any structural deformation even after repeated cycles of macrocyclization. 


\section{Tables}

Table S1. Small-angle X-ray diffraction data fitting into a 3-D hexagonal structure of 1. $q_{\text {calcd }}$ and $q_{\text {obsd }}$ are the scattering vectors of the calculated and the observed reflections for the hexagonal structure with lattice parameters, $\mathrm{a}=4.6 \mathrm{~nm}$ and $\mathrm{c}=4.1$ $\mathrm{nm}$.

\begin{tabular}{ccccc}
\hline $\mathrm{h}$ & $\mathrm{k}$ & $\mathrm{l}$ & $q_{\text {calcd }}\left(\mathrm{nm}^{-1}\right)$ & $q_{\text {obsd }}\left(\mathrm{nm}^{-1}\right)$ \\
\hline 0 & 0 & 1 & 1.523 & 1.518 \\
1 & 0 & 0 & 1.594 & 1.591 \\
1 & 1 & 0 & 2.762 & 2.742 \\
0 & 0 & 2 & 3.032 & 3.034 \\
2 & 0 & 0 & 3.189 & 3.179 \\
1 & 1 & 2 & 4.100 & 4.120 \\
2 & 1 & 0 & 4.213 & 4.216 \\
0 & 0 & 3 & 4.555 & 4.561 \\
1 & 0 & 3 & 4.825 & 4.824 \\
2 & 0 & 3 & 5.564 & 5.583 \\
3 & 1 & 0 & 5.734 & 5.743 \\
1 & 0 & 4 & 6.274 & 6.260 \\
4 & 0 & 1 & 6.544 & 6.547 \\
3 & 2 & 0 & 6.941 & 6.936 \\
\hline
\end{tabular}

Table S2. Characterization of the unit cell of $\mathbf{1}$ from SAXS data and density measurements. $N_{\text {cell }}=$ number of molecules in a unit cell of the hexagonal lattice, calculated according to the following equation: $N_{\text {cell }}=\rho \times V_{\text {cell }} \times N_{A} \div M$, where $M$ is the molecular weight, $\rho$ is density, $N_{A}$ is Avogadro' number, and $V_{\text {cell }}$ is the volume of unit cell defined by $a^{2} \times \sin 60^{\circ} \times c$. The density was measured by flotation in $n$-hexane/ $\mathrm{CCl}_{4}$ mixture solution.

\begin{tabular}{cccccc}
\hline Compound & Density $\left(\mathrm{g} / \mathrm{cm}^{3}\right)$ & $\mathrm{a}(\mathrm{nm})$ & $\mathrm{c}(\mathrm{nm})$ & $\gamma\left({ }^{\circ}\right)$ & $N_{\text {cell }}$ \\
\hline 1 & 0.91 & 4.60 & 4.10 & 60 & 18 \\
\hline
\end{tabular}


Table S3. Molecular size, uptake and substrate conversion of R1, R2, R3 and R4 in single-layered porous nanosheets. The molecular size of the substrates with a constrictive conformation was determined by molecular dynamic simulations in water.

\begin{tabular}{|c|c|c|c|}
\hline Reactant & Size $(\mathrm{nm})$ & Uptake & Conversion \\
\hline R1 & 2.0 & $96.06 \pm 1.12 \%$ & $\sim 100 \%$ \\
\hdashline R2 & 1.9 & $95.21 \pm 0.48 \%$ & $\sim 100 \%$ \\
\hline R3 & 1.3 & - & - \\
\hline R4 & 2.3 & - & - \\
\hline
\end{tabular}

Table S4. Each cycle of uptake and conversion of $\mathbf{R} \mathbf{1}$ in the porous nanosheet structure of $\mathbf{1}$ in water-methanol (3/7, v/v) solutions.

\begin{tabular}{|c|c|c|}
\hline Cycle & Uptake & Conversion \\
\hline 1st & $\sim 96 \%$ & $\sim 100 \%$ \\
\hline 2nd & $\sim 95 \%$ & $\sim 100 \%$ \\
\hline 3rd & $\sim 95 \%$ & $\sim 100 \%$ \\
\hline 4th & $\sim 95 \%$ & $\sim 100 \%$ \\
\hline
\end{tabular}

\section{Supplementary References}

S1. Huang, Z.; Kang, S.-K.; Banno, M.;Yamaguchi, T.; Lee, D.; Seok, C.; Yashima, E.; Lee, M. Pulsating Tubules from Noncovalent Macrocycles. Science 2012, $337,1521-1526$.

S2. Persigehl, P.; Jordan, R.; Nuyken, O. Functionalization of Amphiphilic Poly-(2-oxazoline) Block Copolymers: a Novel Class of Macroligands for Micelle Catalysis. Macromolecules 2000, 33, 6977-6981.

S3. Wang, X.; Yan, J.; Zhou, Y. Surface Modification of Self-Assembled One-Dimensional Organic Structures. J. Am. Chem. Soc. 2010, 132, 1587215874. 
S4. Yuan, W. Z.; Yu, Z.-Q.; Lu, P.; Deng, C.; Lam, J. W. Y.; Wang, Z.; Chen, E.-Q.; Ma, Y.; Tang, B. Z. High Efficiency Luminescent Liquid Crystal: Aggregation-Induced Emission Strategy and Boriented Mesomorphic Structures. J. Mater. Chem. 2012, 22, 3323-3326.

S5. Kozaki, M.; Ninomiya, Y.; Suzuki, S.; Okada, K. Allosteric Regulation of the Ligand-Binding Ability of Zn-porphyrin by Metal Complexation. Tetrahedron Letters 2013, 54, 3658-3661. 